\title{
Modeling and Analysis of Quantum Well Structures in GaNInGaN Light Emitting Diodes
}

\author{
Lekha Kuchipudi
}

Follow this and additional works at: https://researchrepository.wvu.edu/etd

\section{Recommended Citation}

Kuchipudi, Lekha, "Modeling and Analysis of Quantum Well Structures in GaNInGaN Light Emitting Diodes" (2014). Graduate Theses, Dissertations, and Problem Reports. 6019.

https://researchrepository.wvu.edu/etd/6019

This Thesis is protected by copyright and/or related rights. It has been brought to you by the The Research Repository @ WVU with permission from the rights-holder(s). You are free to use this Thesis in any way that is permitted by the copyright and related rights legislation that applies to your use. For other uses you must obtain permission from the rights-holder(s) directly, unless additional rights are indicated by a Creative Commons license in the record and/ or on the work itself. This Thesis has been accepted for inclusion in WVU Graduate Theses, Dissertations, and Problem Reports collection by an authorized administrator of The Research Repository @ WVU. For more information, please contact researchrepository@mail.wvu.edu. 


\title{
Modeling and Analysis of Quantum Well Structures in GaN/InGaN Light Emitting Diodes
}

\author{
by \\ Lekha Kuchipudi \\ Thesis submitted to the \\ Benjamin M. Statler College of Engineering and Mineral Resources \\ at West Virginia University \\ in partial fulfillment of the requirements for the degree of \\ Master of Science \\ in \\ Electrical Engineering \\ Lawrence A. Hornak, Ph.D., Chair \\ Dimitris Korakakis, Ph.D. \\ Jeremy Dawson, Ph.D.
}

Lane Department of Computer Science and Electrical Engineering

Morgantown, West Virginia

2014

Keywords: Quantum Well, Energy Levels, Light Emitting Diodes, EL Spectra Copyright 2014 Lekha Kuchipudi 


\section{Abstract \\ Modeling and Analysis of Quantum Well Structures in GaN/InGaN Light Emitting Diodes}

\section{Lekha Kuchipudi}

In recent years, there has been great research interest in the development of efficient Group IIINitride Light Emitting Diodes (LEDs) due to the range of wavelengths covered by this material family. Quantum wells play a significant role in the performance of these optoelectronic devices as they enable tuning in emission wavelength and color of light through variation in composition and width of the well.

The time and cost associated with iterative development and optimization of such new semiconductor devices through physical materials growth and device fabrication can be prohibitive to full exploration of potential design spaces. Technology Computer Aided Design (TCAD) simulation tools have emerged allowing engineers to explore design alternatives at an early stage. However simulations, especially for new materials and devices exhibiting quantum effects, must reliably model material and device physics and produce results that correspond to those that would have been achieved experimentally. In this thesis, tiberCAD, Crosslight APSYS and Synopsys tools have been evaluated and used to design and analyze components of, as well as complete Multiple Quantum Well LEDs. The effect of variations of mole fraction, well width, and bias on Energy Levels and Electroluminescence (EL) has been examined. Simulation results have been compared with theory and measurements from grown and fabricated GaN/InGaN Multiple Quantum Well LEDs. 


\section{Acknowledgements}

My graduation life in West Virginia University has been an educating and wonderful journey. I loved every moment of my grad life, which included challenging coursework, research and joyful instances with friends. It is now time to acknowledge all of the individuals and sources that have been a crucial influence throughout my graduate career.

First of all, I owe my deepest gratitude to my advisor Dr. Lawrence A. Hornak, for his continuous encouragement and guidance through my master's. This thesis would not have been possible without his intellectual support and the physical resources he made available to me, that made my master's an enriching experience. All the valuable discussions and the training he provided will benefit me throughout my career. I strongly believe that my potential as a professional engineer has increased multifold during my association with Dr. Hornak.

I am very much grateful to my committee members Dr. Dimitris Korakakis and Dr. Jeremy Dawson for their encouragement, technical discussions and feedback. Immense thanks to my lab mates Srinitya Musunuru, Joshua Justice, Anand Kadiyala, Vishal Narang and Sujan Kasani for their help and knowledge exchange in this project.

I owe my sincere thanks and dedicate this thesis to my parents Kuchipudi Venkateswara Rao and Kuchipudi Poornima Rani, for all their support and encouragement through my childhood. I would also like to thank my aunt Sirisha Guttikonda and uncle Kishore Gonuguntla for their guidance and moral support.

Special thanks to my friends Sai Praneeth, Pallavi Anipindi, Sneha Kashtala, Jigeesha Karangula, Harika Bharthavarapu, Neeharika Voddepalli, Bhargavi Thotakura, Kavitha Parupati, Sravanthi Nalamalapu, Savan Suri, Saurabh Chaudhari, Anjana Devineni and Sarika Vanapalli for all their support and encouragement during my stay away from home.

Last but not the least I thank lord almighty for blessing me with such wonderful people in my life. 


\section{Table of Contents}

$\begin{array}{lll}\text { Acknowledgements } & \text { iii }\end{array}$

List of Figures $\quad$ v

List of Tables $\quad$ vi

Notations vii

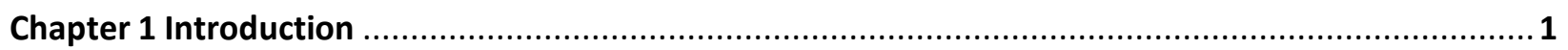

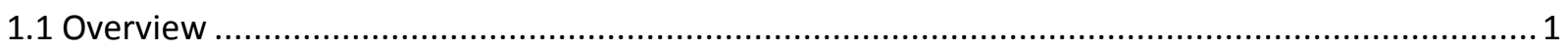

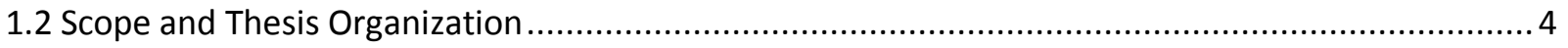

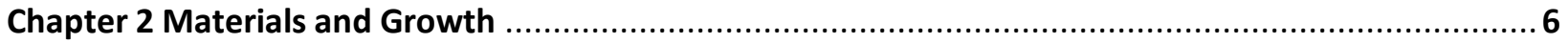

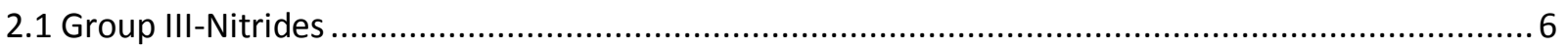

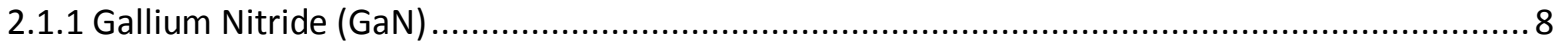

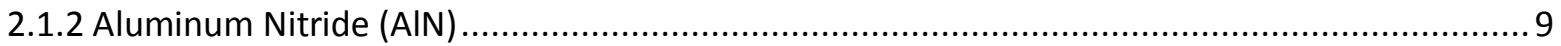

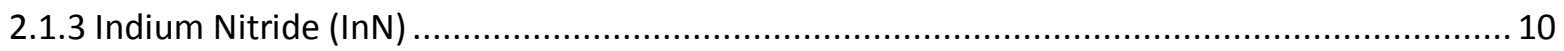

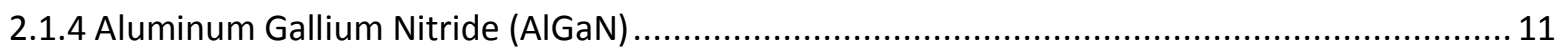

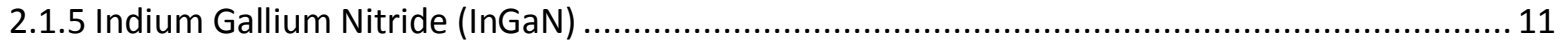

2.2 Polarization Effects in Group III-Nitride Heterostructures ...................................................... 12

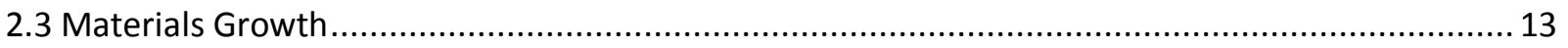

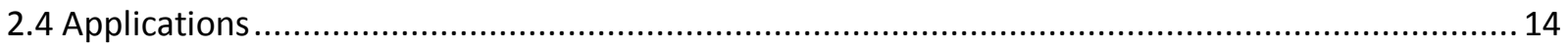

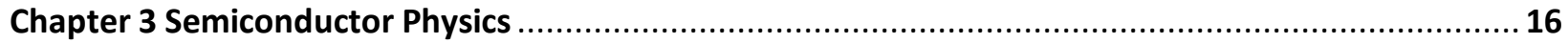

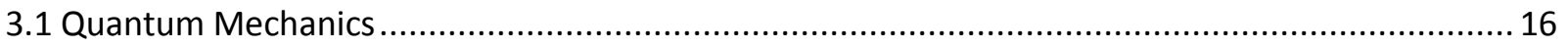

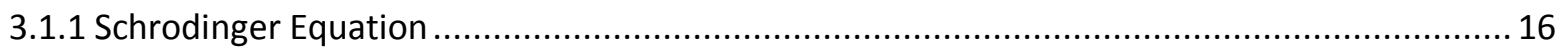

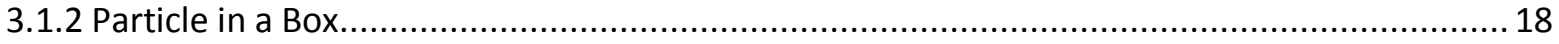

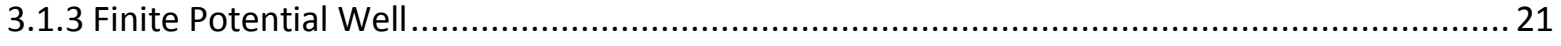

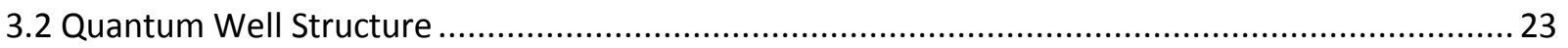

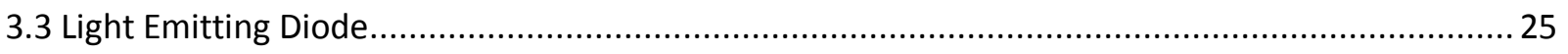

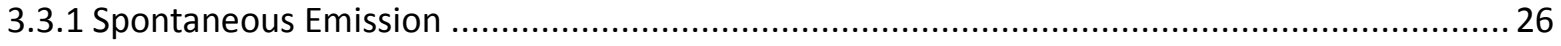

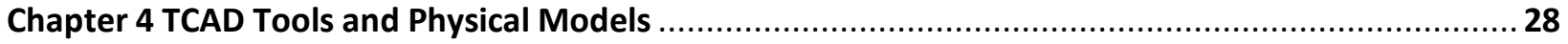

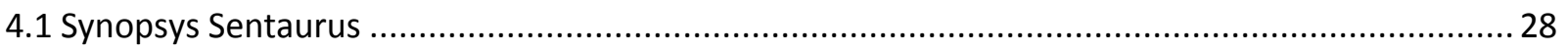

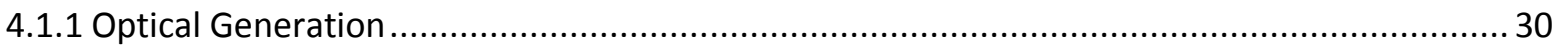

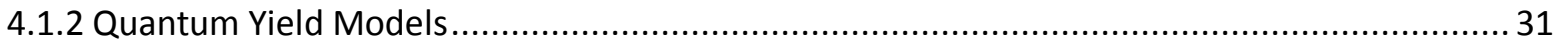




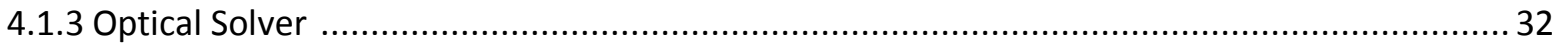

4.1.4 Complex Refractive Index Model ............................................................................... 34

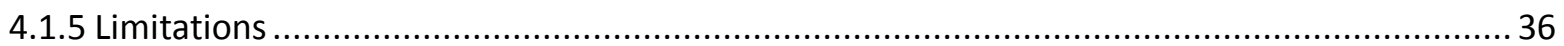

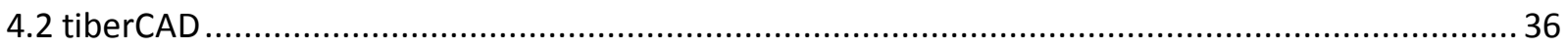

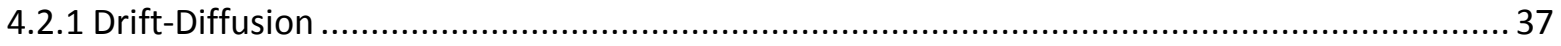

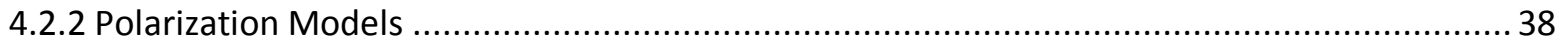

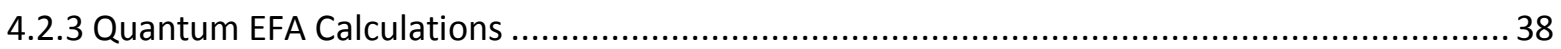

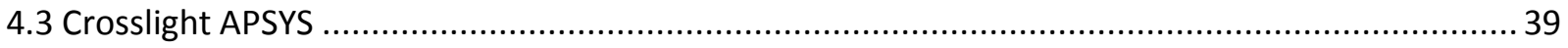

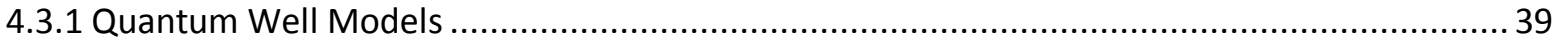

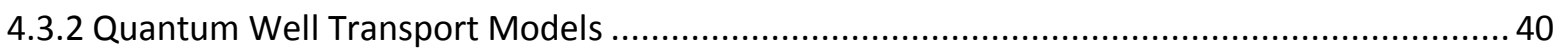

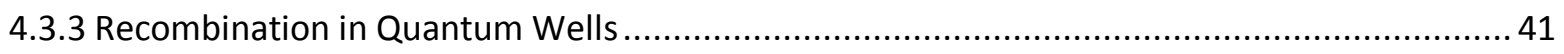

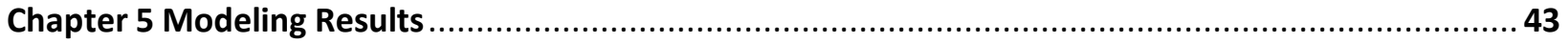

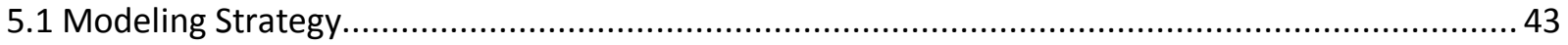

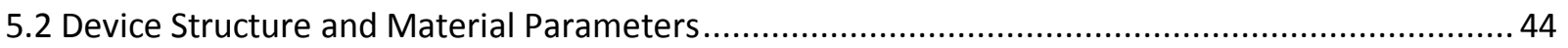

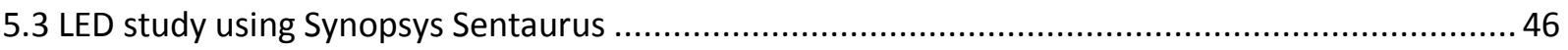

5.4 Study of Quantum Properties of a Single GaN/InGaN QW (1D) using tiberCAD ......................... 49

5.5 Study of Influence of Quantum Well on LED Characteristics using Crosslight APSYS..................... 54

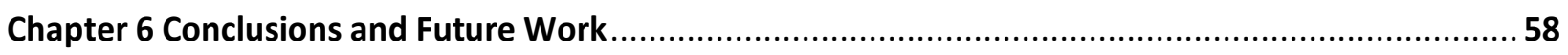

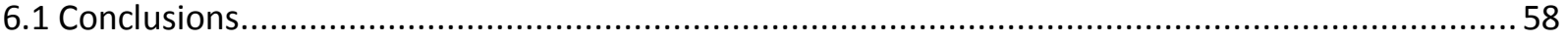

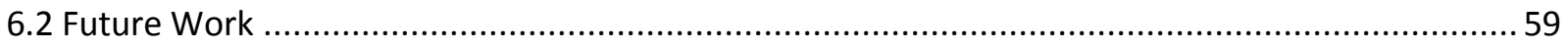

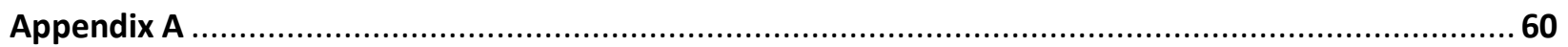

Design of Multiple Quantum Well LED Devices using Sentaurus Workbench ............................... 60

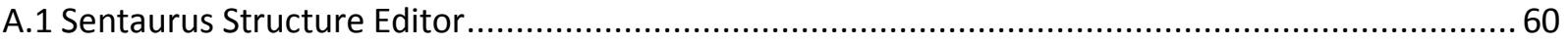

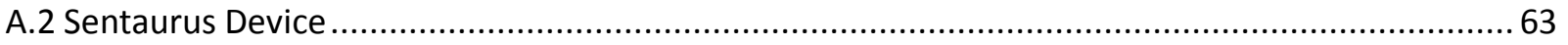

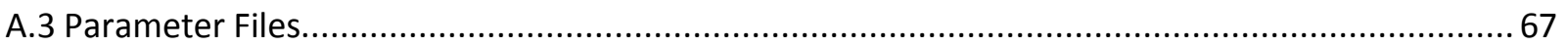

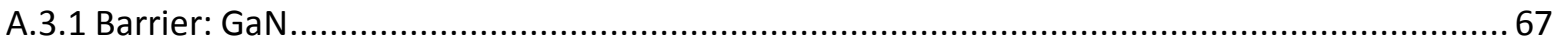

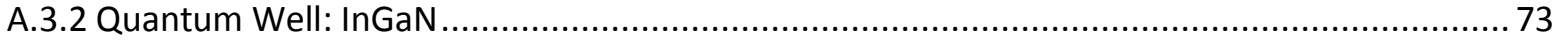

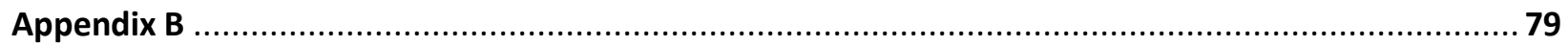

Study of Quantum Properties of a Single GaN/InGaN QW using tiberCAD Tool........................... 79

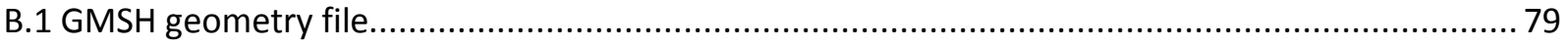

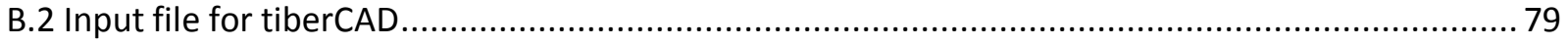

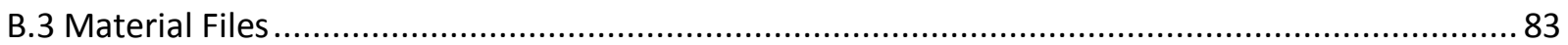




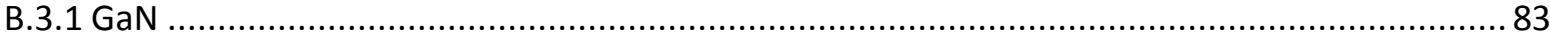

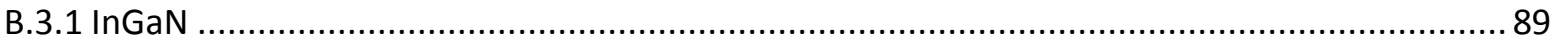

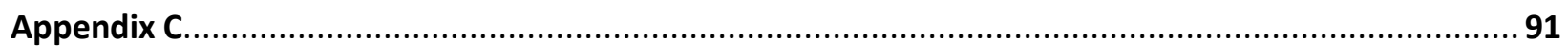

Study of influence of quantum wells on LED characteristics using Crosslight APSYS software ..........91

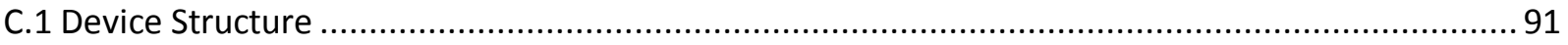

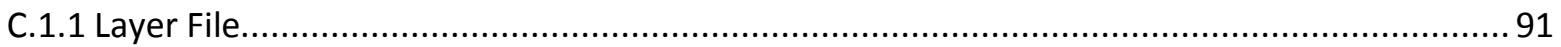

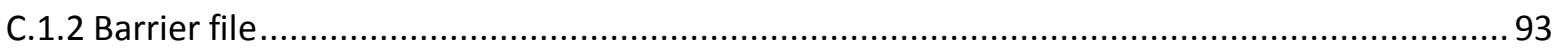

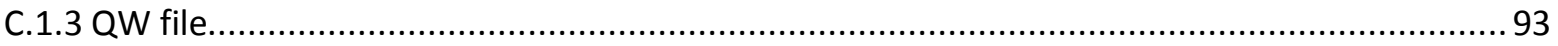

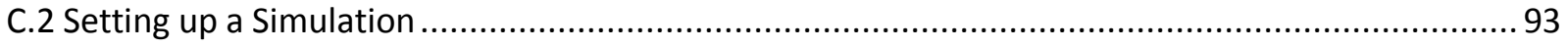

$\begin{array}{lr}\text { References } & 95\end{array}$ 


\section{List of Figures}

Figure 1.1: LED Applications (NIOSH [3], Thermos [4], Armando Emanuel Roggio [5], Philips [6]).......1

Figure 1.2: InGaN/GaN MQW Green LED (Sun X, Li D, Song H [7])............................................. 2

Figure 1.3: Hierarchy of technology CAD tools from the process level to circuits (Luciano Lavagno,

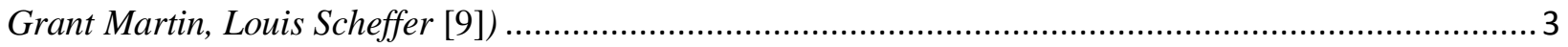

Figure 1.4: Major TCAD Device Simulation Tools ....................................................................... 4

Figure 2.1: Energy bandgap versus lattice constant of group III-nitride semiconductors (E. Fred Schubert

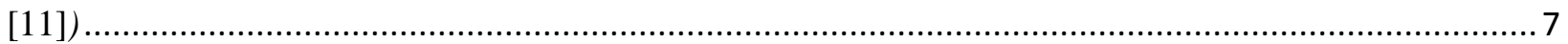

Figure 2.2: (a) Zinc-blende Structure and (b) Wurtzite Structure (Won Seok Lee [13]).......................... 8

Figure 2.3: Wurtzite structure of GaN with different polarities (Ga- and N-faced) (O Ambacher [17]) .....9 Figure 2.4: Surface charges and direction of internal electric field and polarization field for spontaneous and piezoelectric polarization in III-nitrides for Ga- and N-face orientation (Won Seok Lee [13]).......... 13

Figure 2.5: Picture of the Aixtron 200/RF-S MOVPE system used for the growth of all III-nitride

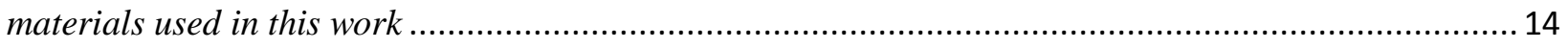

Figure 2.6: (a) Cross section of an III-V LED device (b) advanced III-V LEDs ................................... 15

Figure 2.7: Schematic of HEMT epitaxial structure with location of the 2D electron gas shown as a red

Figure 3.1: Infinite Potential Well (Benjamin D. Esham [37]).......................................................... 18

Figure 3.2: (a) Wave functions and energy levels in an infinite square potential well (R. A. Serway, J.

John W. Jewett [39]) (b) Probability distribution of a particle in a box (Prof. Delmar Larsen [36])........ 20

Figure 3.3: Finite Potential Well (chegg.com [41])...................................................................... 21

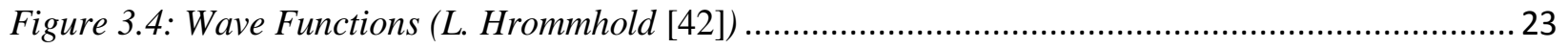

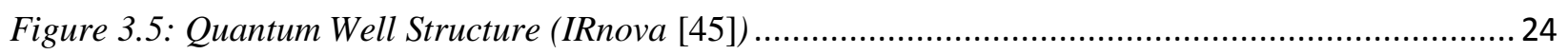

Figure 3.6: (a) Multiple Quantum Well Blue LED (b) WVU Fabricated LED ..................................... 24

Figure 3.7: LED circuit and band diagram (S-kei [48]) .............................................................. 25

Figure 3.8: Electromagnetic Spectrum (Aladdin LED Lights \& Lamps [49]) ........................................ 25

Figure 3.9: Spontaneous Emission (IImari Karonen [50])............................................................. 26

Figure 3.10: Theoretical emission spectrum of an LED (E. Fred Schubert [11]) ..................................2 27

Figure 4.1: Optical generation rate as a function of depth (Synopsys [59]) ......................................... 31

Figure 4.2: Wave amplitudes in a layered medium and transfer matrices connecting them (B. E. A. Saleh,

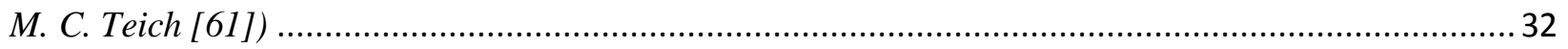

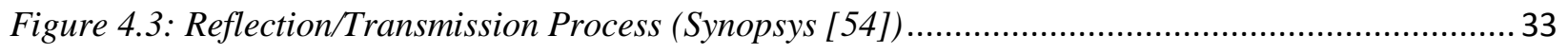

Figure 4.4: Quantum well models in Crosslight (Crosslight [65]) ........................................................ 40

Figure 4.5: Discretization of quantum well to handle the physics of quantum well transport (Zbigniew

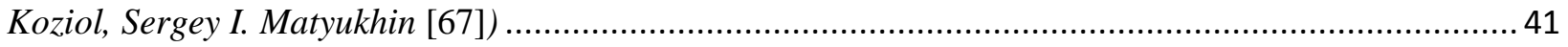

Figure 5.1: TCAD Model Design Flow .................................................................................. 43

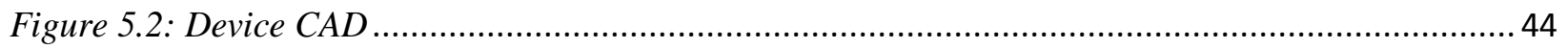

Figure 5.3: LED structure employed for modeling ........................................................................ 45

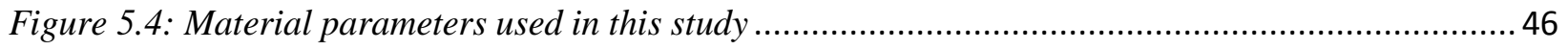

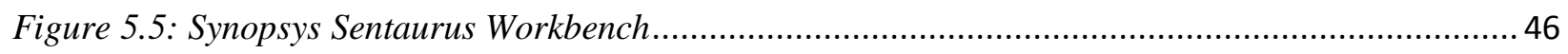


Figure 5.6: Structure and Band Diagram of the LED device plotted along the vertical direction ............ 47

Figure 5.7: Structure used to study optical characteristics ................................................................ 48

Figure 5.8: (a) Photoluminescence Spectrum (b) Experimental PL Spectrum from Literature (Zhe Chaun,

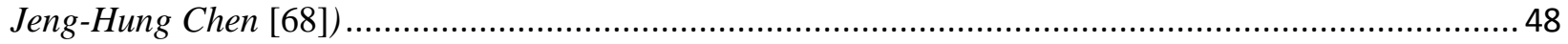

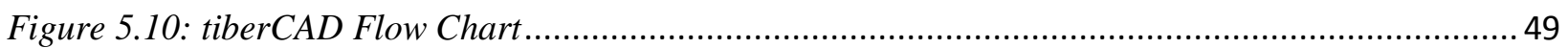

Figure 5.11: (a) Single GaN/InGaN QW structure used for modeling (b) Band structure at equilibrium.50

Figure 5.12: Band structure of a SQW at different biases.................................................................. 50

Figure 5.13: Energy levels confined in conduction and valence bands at equilibrium........................... 51

Figure 5.14: Verification and Validation (Yuan Zhi-liang, Xu Zhong-ying [69])................................. 52

Figure 5.15: Influence of quantum well widths on energy levels of a single quantum well at forward bias

Figure 5.16: Influence of quantum well molefraction on energy levels of a single quantum well at forward

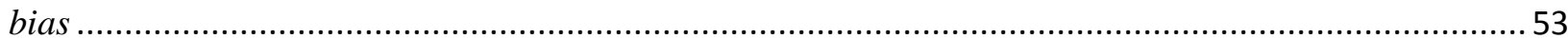

Figure 5.17: Crosslight APSYS Flow Chart ..................................................................................... 54

Figure 5.18: Band diagram and Wavefunctions of InGaN LED (a) Non-polarized (b) Polarized .............55

Figure 5.19: Comparison of Experimental and Simulation Electroluminescence Spectra results ............ 56

Figure 5.20: Influence of quantum well molefraction on EL Spectra .............................................. 57 


\section{List of Tables}

Table 2.1: Some basic parameters of III-Nitride materials of Wurtzite structure at 300k (Hadis Morkoc [18], Ioffe Physico [24], Ashraful Ghani Bhuiyan [23], Shih-Chieh Lin [25]).................................... 10

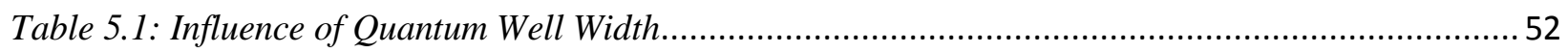

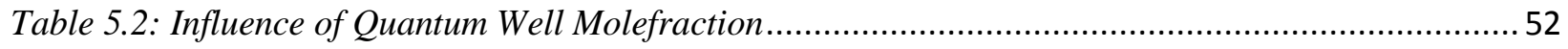

Table 5.3: EL Spectra experimental and simulation comaprison ...................................................... 56

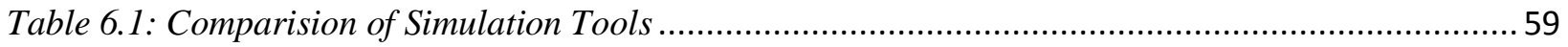




\section{Notations}

We use the following notation and symbols throughout this thesis

LED : Light emitting diode

MQW : Multiple quantum well

SQW : Single quantum well

TCAD : Technology Computer-Aided Design

MOCVD : Metal Organic Chemical Vapor Deposition

XRD : X-Ray Diffraction

EL : Electroluminescence

PL : Photoluminescence 


\section{Chapter 1 Introduction}

This chapter reviews the relevant scientific development of light emitting diodes over the years and the evolution of TCAD modeling tools. Then a brief discussion of the thesis organization is presented.

\subsection{Overview}

There has been a long-standing interest in the development of solid-state light-emitting devices as they have proved to be more efficient than the conventional tungsten filament light bulbs. For over 30 years, they have been used in various areas of applications such as in communication systems, lightning applications and the automobile industry. Figure 1.1 shows several industrial applications of LEDs. These applications have placed stringent demands on improvements in the performance of LEDs. Specific expertise such as understanding the material, structure and composition thoroughly is needed in order to obtain precise and reproducible results. In summary, LEDs have gone from infancy to adolescence resulting in a constant pressure on manufacturers to produce higher performance devices in a shorter time [1] [2].

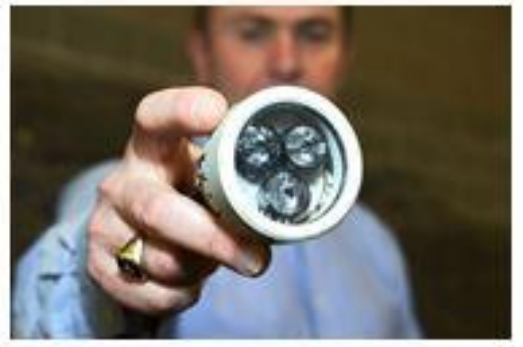

Mining Operations

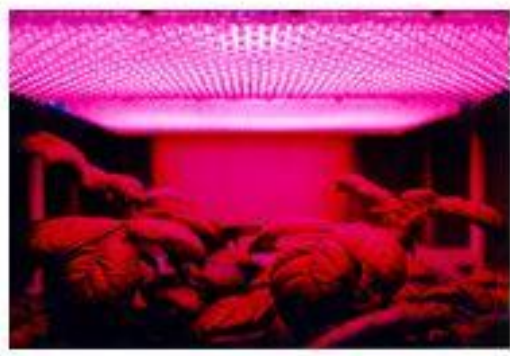

Space Crops

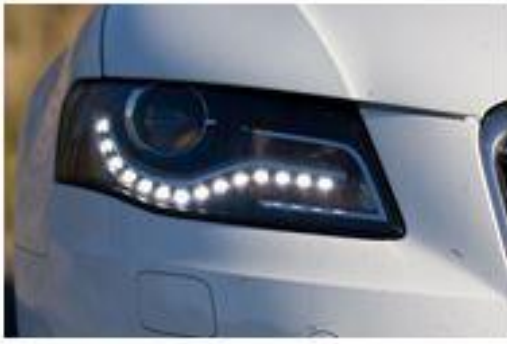

Automotive Applications

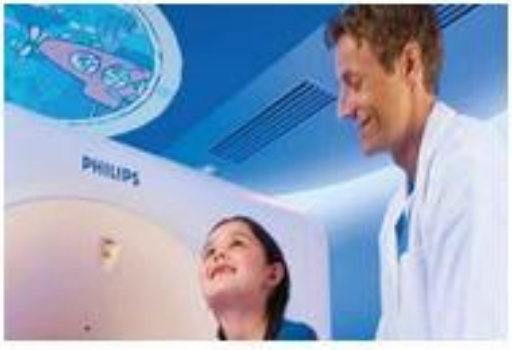

Healthcare

Figure 1.1: LED Applications (NIOSH [3], Thermos [4], Armando Emanuel Roggio [5], Philips [6]) 
The need for efficient Light Emitting Diodes drives research into new direct-gap semiconductors to act as the active materials. These new structures exploit the principles of quantum confinement to engineer electronic states with custom-designed properties. Figure 1.2 shows a Group III-Nitride MQW LED.

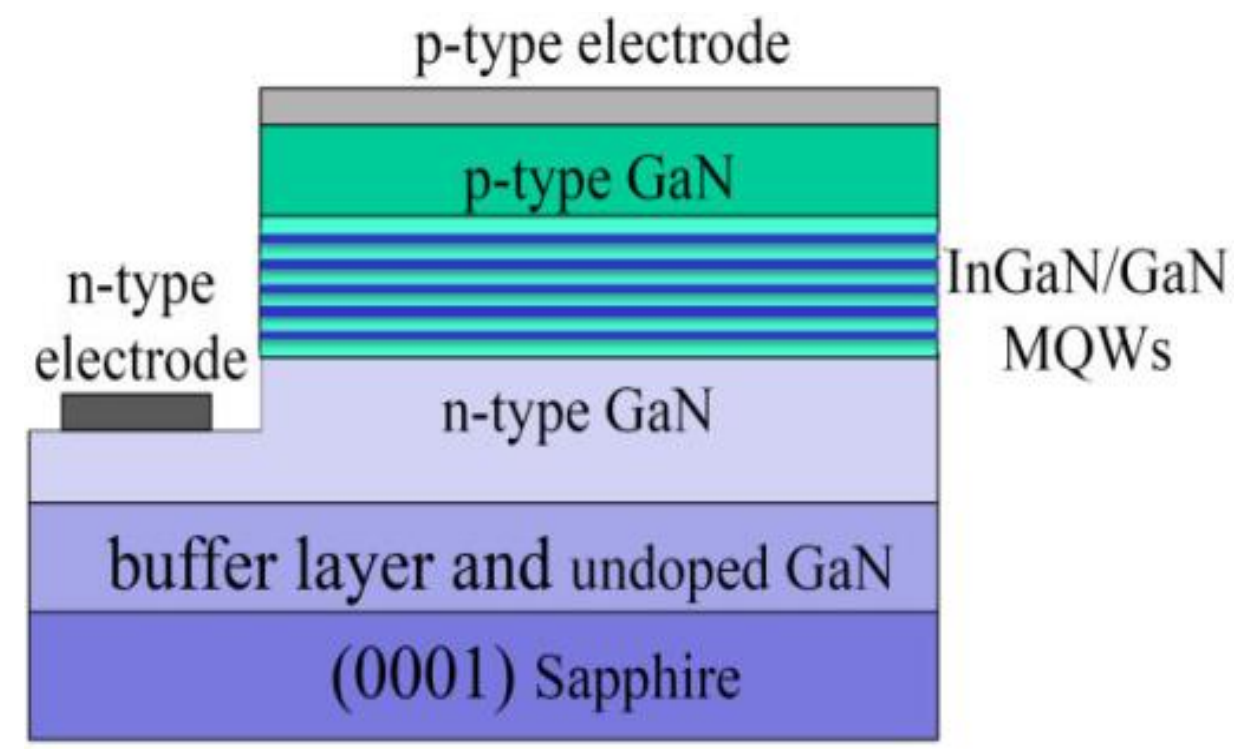

Figure 1.2: InGaN/GaN MQW Green LED (Sun X, Li D, Song H [7])

The time and cost associated with iterative development and optimization of such new semiconductor devices through physical materials growth and device fabrication can be prohibitive to full exploration of potential design spaces. Technology Computer Aided Design (TCAD) simulation tools have emerged allowing engineers to explore design alternatives at an early stage.

TCAD refers to the use of computer simulations to develop and optimize semiconductor devices. TCAD offers significant savings in time and money to semiconductor vendors. According to the International Technology Roadmap for Semiconductors, technology development costs can be reduced as much as 40 percent by using TCAD. TCAD tools are available at different levels for different applications.

- Process CAD: modeling of the fabrication

- Device CAD: modeling of the device

- ECAD: compact models to capture electrical behavior of devices 
Multiple commercially and openly available TCAD simulation tools are available. In the following a short overview of the evolution of technology computer-aided design (TCAD) modeling tools will be given. The following overview reflects only the main tools. The history of commercial TCAD dates back to late 1960s dominated by IC development. In 1979, researchers from Stanford University under the guidance of Professors Dutton and Plummer formed a company Technology Modeling Associates (TMA) to produce commercial TCAD software tools. SUPREM-III and PISCES are the most famous tools released by TMA. SUPREM-III is a one-dimensional process simulator used in the manufacture of silicon integrated circuits or discrete devices. PISCES is a two-dimensional simulator and implements advanced physical models and improved numerical techniques for a 2D device analysis. TSUPREM4 and MEDICl are other versions of TMS tools. But, later SILVACO licensed these programs from Stanford University too and offered a commercial alternative (ATHENA and ATLAS). The third major TCAD vendor was Integrated Systems Engineering (ISE). Their equivalent product offerings were DIOS and DESSIS. TMA was later acquired by Avant, which was then acquired by Synopsys. Recently Synopsys acquired their main competitor ISE, which leaves only Synopsys and Silvaco as the main competitors in the market. Synopsys owns now more than $80 \%$ of the market share, which results in a market situation close to a monopoly [8].

Extrinsic (and Layout)

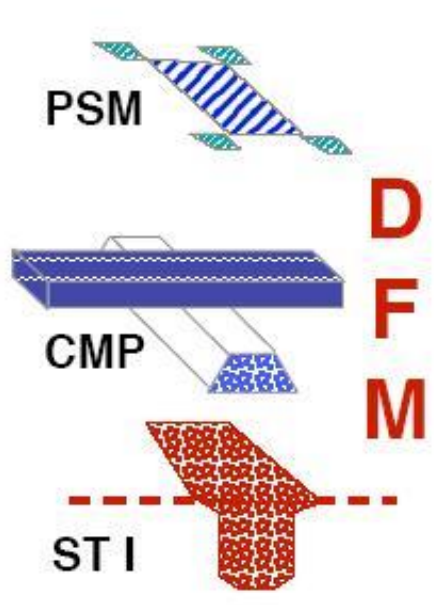

Intrinsic (active/passive devices)
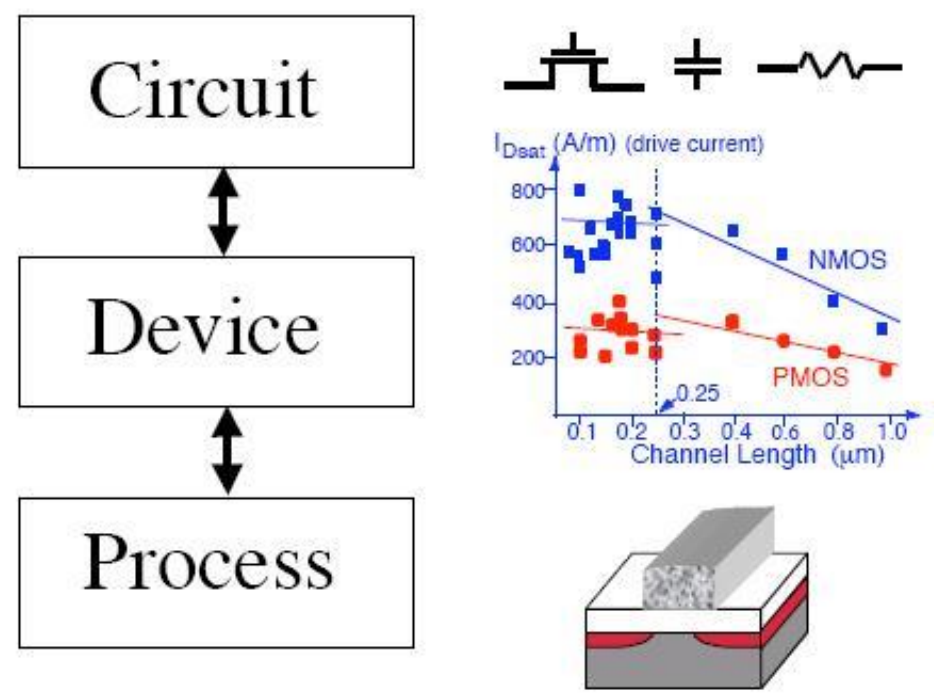

Figure 1.3: Hierarchy of technology CAD tools from the process level to circuits (Luciano Lavagno, Grant Martin, Louis Scheffer [9]) 
In this thesis, Device CAD tools were used to study multiple quantum well light emitting diodes. However simulations, especially for new materials and devices exhibiting quantum effects, must reliably model material and device physics and produce results that correspond to those that would have been achieved experimentally. The earlier TCAD tools from TMA and Synopsys do not support quantum well transport models for active devices for now. So, tiberCAD 2.0 and Crosslight APSYS device simulation tools were used to study the quantum well behavior in light emitting diodes as they support quantum well transport and carrier scattering models.

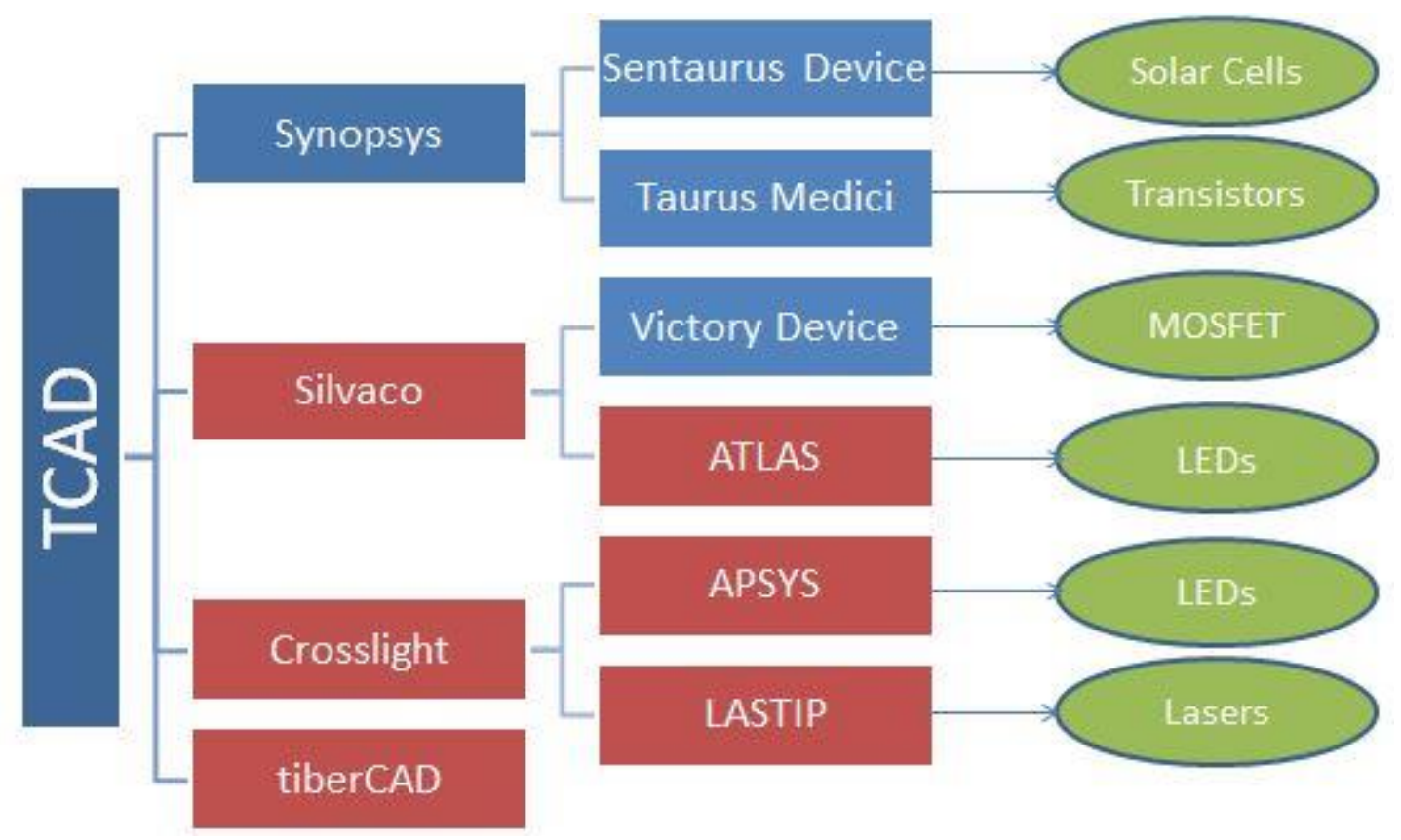

Figure 1.4: Major TCAD Device Simulation Tools

\subsection{Scope and Thesis Organization}

This thesis mainly focuses on design and analysis of light emitting diodes using TCAD tools that support quantum well models. In this thesis, tiberCAD, Crosslight APSYS and Synopsys tools have been evaluated and used to design and analyze components of, as well as complete Multiple Quantum Well LEDs. The effect of variations of mole fraction, well width, and bias on energy levels in a quantum well and electroluminescence (EL) characteristics have been examined. Simulation results have been compared with theory and measurements from grown and fabricated GaN/InGaN Multiple Quantum Well LEDs. 
This thesis is organized as follows: Chapter 2 presents in detail, an overview of semiconductor materials used in this study. The significance of material properties of group III-nitrides and their ternary compounds are discussed. Then, the growth and applications of group III-nitride semiconductors are reviewed.

Chapter 3 presents a discussion of quantum well physics and quantum well structures in light emitting diodes. A related phenomenon - particle in a box model is reviewed in the physics section. It is followed by the discussion of physical effects of quantum wells on light emitting diode characteristics.

Chapter 4 gives an overview of the simulation tools and the physical models used for the comprehensive simulation of semiconductor multiple quantum well optoelectronic devices with an in detail explanation. The simulation templates used for this study are Synopsys TCAD Sentaurus Workbench Version H-2013.03, tiberCAD 2.1.0 and Crosslight APSYS.

Chapter 5 presents a systematic study of the design and analysis of multiple quantum well LEDs to investigate the influence of quantum wells on the performance of the LEDs. Several TCAD tools and their effectiveness in analyzing devices have been evaluated.

Finally, Chapter 6 summarizes the findings and conclusions of this works. 


\section{Chapter 2 Materials and Growth}

This chapter presents in detail, an overview of semiconductor materials used in this study. The significance of material properties of group III-nitrides and their ternary compounds are discussed. Then, the growth and applications of group III-nitride semiconductors are reviewed.

\subsection{Group III-Nitrides}

III-Nitride based semiconductor materials (Al, In, Ga)N are excellent wide band gap semiconductors with unique properties which make them very suitable for modern electronic and optoelectronic applications [10]. The group III nitrides family have a bandgap range from a possible value of 0.6-0.7eV [10] for $\operatorname{InN}, 3.42 \mathrm{eV}$ [10] for GaN, and 6.0eV [10] for AIN at room temperature. Figure 2.1 shows the bangap energy of the III-N's as a function of the a-lattice parameter. Alloys such as InGaN and AlGaN may span a continuous range of direct-band-gap energies, covering the spectral range from UV to visible and to infrared [10].

The group III-Nitrides can exist in both wurtzite and zinc blende structures. Under ambient conditions, the nitride family are considered to be more thermodynamically stable in wurtzite crystalline structure. GaN and InN can also exist as stabilized zinc blende structures when epitaxially grown on cubic substrates such as Silicon (Si), Silicon Carbide ( $\mathrm{SiC}$ ) and Gallium Arsenide (GaAs). Crystallographically, the zinc-blende structure and wurtzite structure are closely related, but are opposed by the stacking sequence of close packed planes. The two structures are shown in Figure 2.2. The wurtzite structure is a member of the hexagonal system characterized by two lattice parameters a and $c$, where a represents the edge length of the basal hexagon and c refers to height of the hexagonal unit cell. Whereas, the zinc blende structure is a cubic unit cell composed of two interpenetrating facecentered sublattices shifted by one quarter of a body diagonal [10]. 


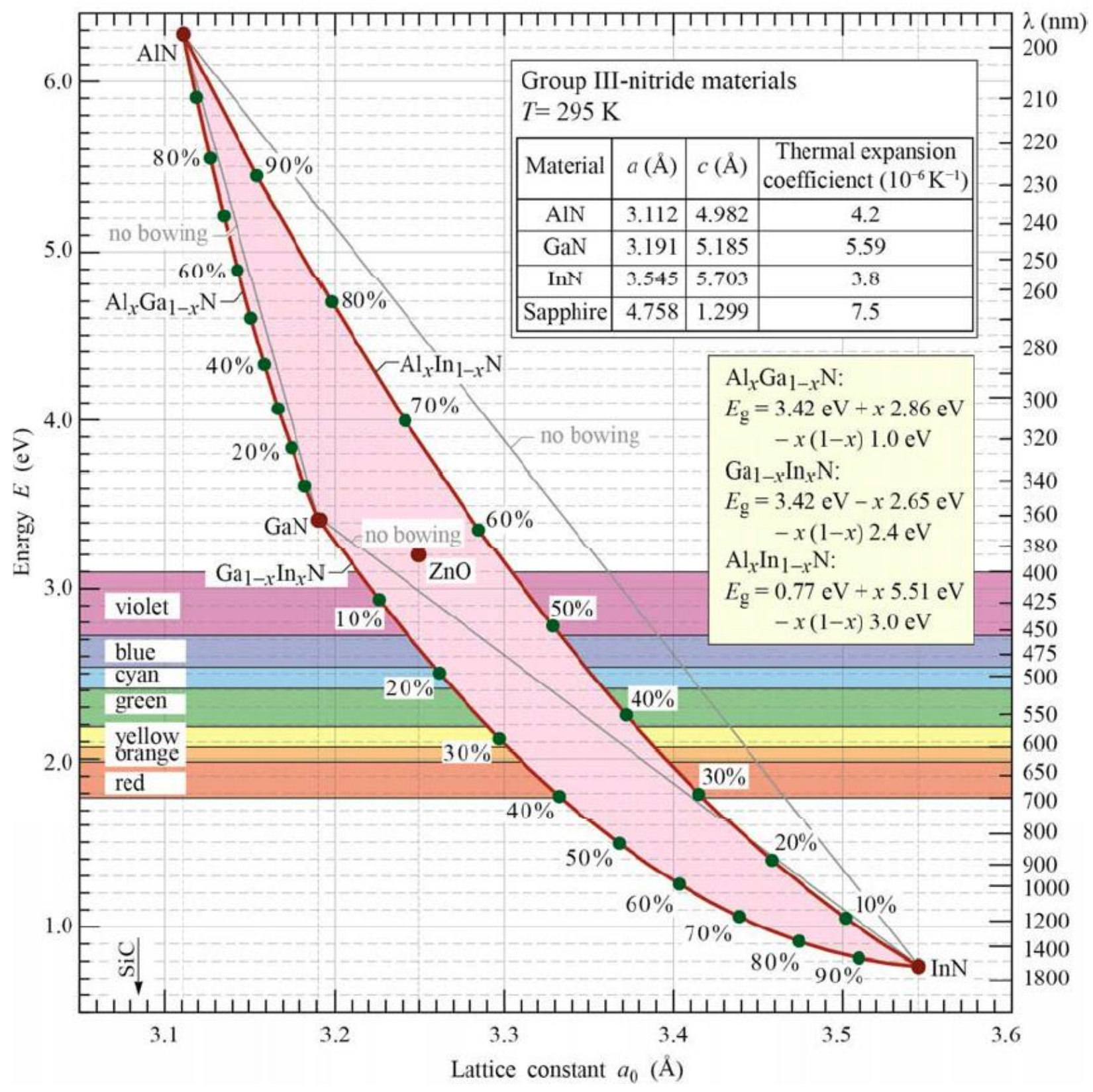

Figure 2.1: Energy bandgap versus lattice constant of group III-nitride semiconductors (E. Fred Schubert [11])

Due to the range of wavelengths covered by this material family, the nitride system is made attractive for various optoelectronic applications such as light-emitting diodes (LEDs), laser diodes (LDs) and UV detectors. It is expected that the III-Nitrides-based LEDs will soon replace the incandescent light bulbs leading to higher quality lights at increasingly affordable prices [12]. Further advancement in 
developing these materials for several applications is expected in the next few years by better understanding and precise measurements of material properties.

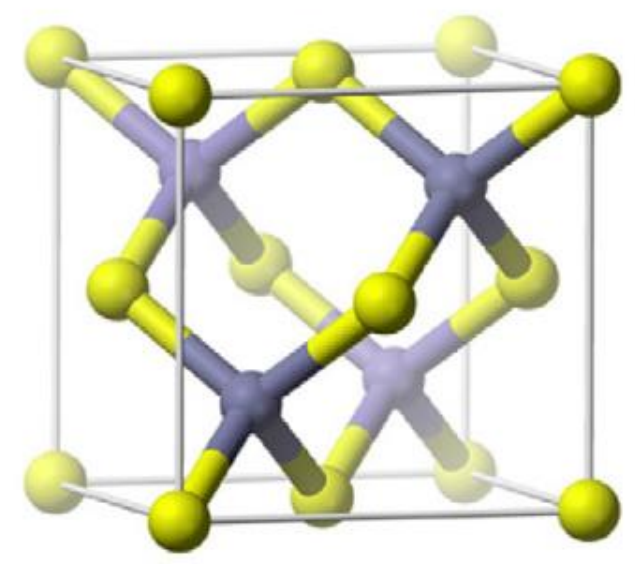

(a) Zinc-blende structure

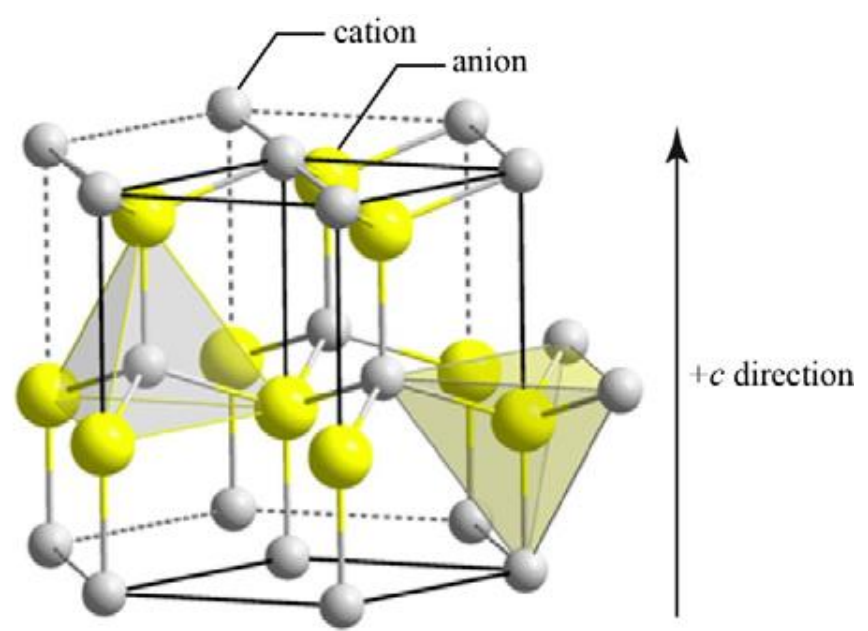

(b) Wutzite structure

Figure 2.2: (a) Zinc-blende Structure and (b) Wurtzite Structure (Won Seok Lee [13])

The material properties of the III-nitride materials used in this work are discussed in the following next sections along with the material growth and the applications in which they are incorporated in today's world.

\subsubsection{Gallium Nitride (GaN)}

GaN is a group III-V direct bandgap semiconductor material with attractive physical characteristics for applications in high frequency devices and high-output devices [14]. Due to its unique material and electronic properties, GaN will dominate silicon power devices in speed, temperature and power handling. Also, GaN offers ten times higher electrical breakdown characteristics, three times the bandgap, and exceptional carrier mobility when compared to silicon [15]

GaN exists in both wurtzite and zinc blende crystal structures. It has a direct bandgap in both the structures with a typical bandgap value of 3.4eV. Different polarities of wurtzite GaN are shown in Figure 2.3. GaN is typically grown as $n$-type semiconductor and the p-type material is not as conducting as $n$ type. Silicon and magnesium are used as n-type and p-type dopants, however GaN crystal growth is altered due to the tensile stresses introduced by these dopants [16]. Due to the larger bonding strength between atoms in the GaN semiconductor, it is thermally, chemically and mechanically robust in nature 
[14]. Table 1.1 shows the basic parameters of III-Nitride materials i.e. GaN, AIN and InN at room temperature.

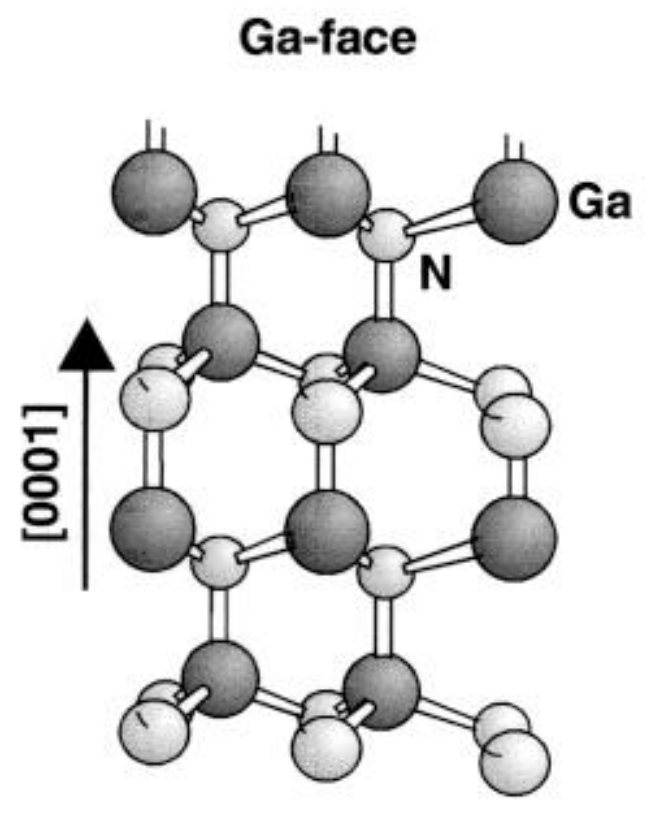

Substrate

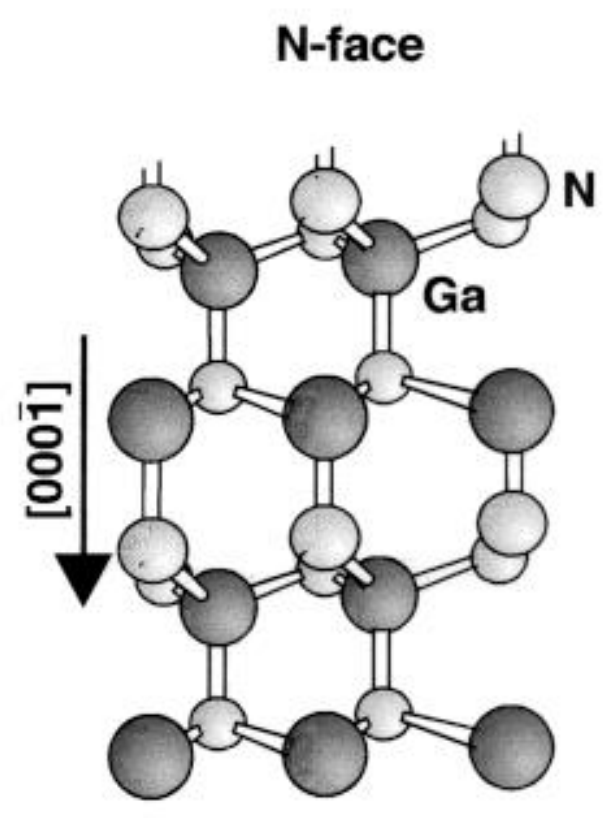

Substrate

Figure 2.3: Wurtzite structure of GaN with different polarities (Ga- and $N$-faced) (O Ambacher [17])

\subsubsection{Aluminum Nitride (AlN)}

AIN is the second most important binary material in the III-N material family next to GaN for electronic applications. It is a wide band gap semiconductor material with band gap energy about 6.2eV at room temperature and is mostly used as a ternary alloy formed with GaN i.e. $A l_{x} G a_{1-x} N$ [18]. Due to its high thermal conductivity, it is a potential for application in microelectronics [19].

AlGaN alloy is mostly used as an electron blocking layer (EBL) in many LED structures to improve the confinement capability of electrons within the active region. Recent studies on LED efficiency indicate that LED structures with an EBL show higher external quantum efficiencies than LEDs without an EBL. However, an efficiency droop was observed at higher injection currents and also EQE was observed to be higher in LED structures without an EBL [20]. 
AIN is also used as a nucleation layer during the growth of GaN films on a substrate. The primary purpose of nucleation layer is to obtain high quality, uniform layers during the growth sequence. Incorporation of nucleation layer improves the surface morphology by avoiding the lattice mismatch issue [21].

\subsubsection{Indium Nitride (InN)}

During the last few years the interest in the $\operatorname{InN}$ has been remarkable. The importance of InN and its ternary compounds is due to the smaller band gap relative to GaN. Recently, researchers have found out that the bandgap of $\mathrm{InN}$ is $0.7 \mathrm{eV}$ rather than around $2.0 \mathrm{eV}$ as previously believed. This means that the alloys of InN have bandgaps that can span the entire visible spectrum to the ultraviolet. Thus, making InN an important semiconductor in many potential applications such as light emitting diodes (LEDs) and laser diodes (LDs) [22] [23].

However, there are difficulties/challenges associated with the growth of high-quality InN. Low InN dissociation temperature and high equilibrium vapor pressure of nitrogen over the $\mathrm{InN}$ film, and lack of suitable substrate were the major obstacles even with the availability of MOVPE and MBE techniques. Recently, good quality InN films are widely explored and studied with the developments in the growth techniques, MOVPE and MBE, as well as by other growth techniques [23].

The development of InN based heterostructures and devices is still at its initial stage and when compared with other compounds, further research is required in investigating $\ln N$ material properties.

Table 2.1: Some basic parameters of III-Nitride materials of Wurtzite structure at 300k (Hadis Morkoc [18], Ioffe Physico [24], Ashraful Ghani Bhuiyan [23], Shih-Chieh Lin [25])

\begin{tabular}{|c|c|c|c|}
\hline Property & GaN & AlN & InN \\
\hline Lattice constant, a [A] & 3.189 & 3.112 & 3.533 \\
\hline Lattice constant, c [A] & 5.186 & 4.982 & 5.693 \\
\hline Band Gap [eV] & 3.39 & 6.026 & 0.7 \\
\hline Electron affinity [eV] & 4.1 & 0.6 & 4.7 \\
\hline Dielectric constant & 8.9 & 8.5 & 15.3 \\
\hline
\end{tabular}




\subsubsection{Aluminum Gallium Nitride (AlGaN)}

Aluminum Gallium Nitride is an alloy of Aluminum Nitride (AIN) and Gallium Nitride (GaN) with useful semiconductor properties. It is the most important ternary compound, which is often used as barrier material in devices and as an active layer to a lesser extent [18]. The material properties of AIGaN $\left(P_{A_{x} B_{1-x} N}\right)$ can be derived from the following formula (2.1), called Vegard's law [26]:

$$
P_{A_{x} B_{1-x} N}=x \cdot P_{A N}+(1-x) \cdot P_{B N}
$$

where, $x$ is the AIN molar fraction and $P_{A N}, P_{B N}$ represent material properties of AIN and GaN respectively.

The compositional dependence of AIGaN principal bandgap can be calculated from the following empirical expression [18] (2.2) provided that the bowing factor b is known accurately:

$$
E_{g}(x)=x E_{g}(A l N)+(1-x) E_{g}(G a N)-b x(1-x)
$$

where, $E_{g}(A l N)=6.1 \mathrm{eV}, E_{g}(G a N)=3.4 \mathrm{eV}$ and $\mathrm{b}$ is the bowing parameter.

\subsubsection{Indium Gallium Nitride (InGaN)}

InGaN alloy is a mix of Indium Nitride ( $\operatorname{lnN}$ ) and Gallium Nitride (GaN). It is a ternary group III-V direct bandgap semiconductor, which forms the backbone of emitters in wide wavelength range. Apart from its crucial role, there are complexities associated with this ternary such as phase separation and instabilities anomalies due to the great disparity between Ga and In [18]. As in the case of AIGaN, the material properties of this alloy follows the Vegard's law:

$$
P_{A_{x} B_{1-x} N}=x \cdot P_{A N}+(1-x) \cdot P_{B N}
$$

where, $x$ is the $\operatorname{lnN}$ molar fraction and $P_{A N}, P_{B N}$ represent material properties of $\ln N$ and $G a N$ respectively.

InGaN bandgap plays a crucial parameter in designs of any heterostructure utilizing it [18]. The compositional dependence can be calculated using the following empirical expression:

$$
E^{g}{ }_{I n_{x} G a_{1-x} N}=x E^{g}{ }_{I n N}+(1-x) E_{G a N}^{g}-b_{I n G a N} x(1-x)
$$

where, $E_{G a N}^{g}=3.4 \mathrm{eV}, E_{I n N}^{g} \approx 0.7 \mathrm{eV}$ and $\mathrm{b}$ is the bowing parameter. 
Recently, high quality $I n_{x} G a_{1-x} N$ layers were grown using Molecular Beam Epitaxy (MBE) on sapphire substrate. But, high indium content good quality films could not be realized by MOCVD, whereas MBE has allowed it instead [26].

\subsection{Polarization Effects in Group III-Nitride Heterostructures}

Polarization effects play an extremely prominent role in Group III-nitride semiconductors. The most common epitaxial growth direction of group III-nitrides is the c-plane of the wurtzite structure. As a consequence of the noncentrosymmetry of the wurzite structure and the large ionicity factor of the covalent metal-nitrogen bond, a large spontaneous polarization oriented along the hexagonal c-axis is predicted. In addition, group-III nitrides are highly piezoelectric. The strain induced piezoelectric as well as the spontaneous polarizations are expected to be present and to govern the optical and electrical properties of GaN based heterostructures to a certain extent, due to the huge polarization constants which are one of the most fascinating aspects of the nitrides [27]. Both spontaneous and piezoelectric polarizations in a given strain state need to be considered for total polarization $P$

$$
P=P_{s p}+P_{p z}(\varepsilon)
$$

where, $P_{s p}$ is the spontaneous polarization and $P_{p z}(\varepsilon)$ is the piezoelectric polarization as function of inplane strain, $\varepsilon$ [13].

Light emitting diodes based on GaN, AlGaN, and InGaN have already been produced and marketed with great success. It has been well documented that these quantum well structures possess a large redshift in the emission versus absorption spectrum. This has been shown to result from the piezoelectric fields generated by strain in the well layer. These piezoelectric fields result in charge separation and decreased oscillator strength for the quantum well structure, resulting in decreased efficiency for light emitting devices [28]. 


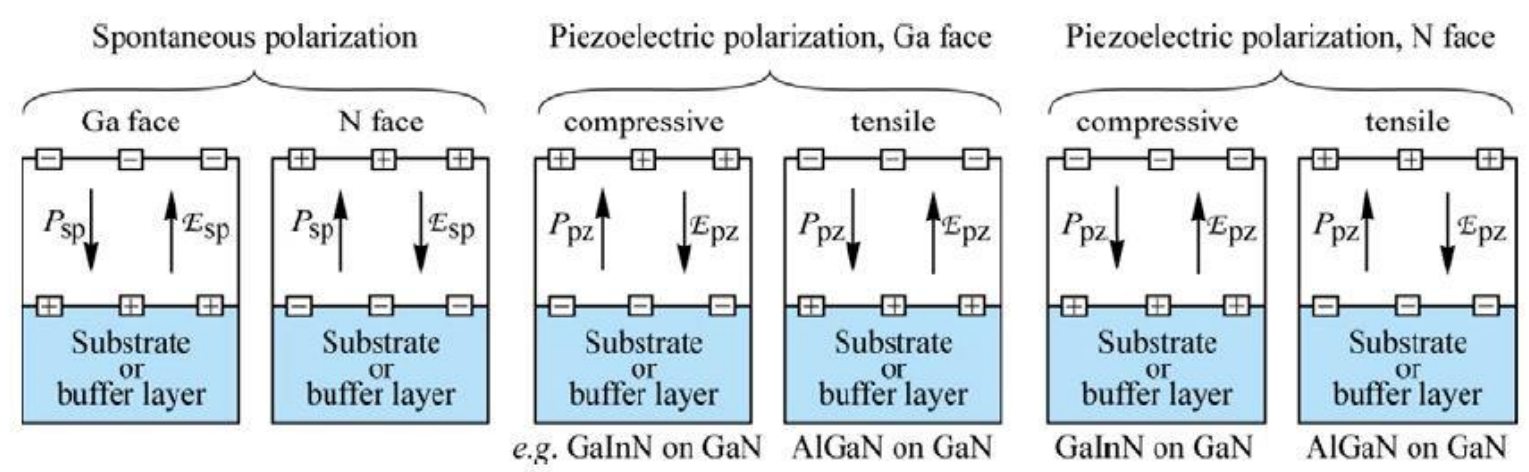

Figure 2.4: Surface charges and direction of internal electric field and polarization field for spontaneous and piezoelectric polarization in III-nitrides for $\mathrm{Ga}$ - and $\mathrm{N}$-face orientation (Won Seok Lee [13])

\subsection{Materials Growth}

Due to the difficulty in maintaining stoichiometry, the growth of compound semiconductors has become more challenging when compared to Si. Especially, group III-V compounds usually have one component more volatile, e.g. As and $\mathrm{P}$ evaporate more readily. Melt growth techniques and epitaxial growth are the generalized techniques used for the growth of compound semiconductors. Bulk crystal growth is possible with melt growth techniques whereas, epitaxy technique allows the growth of multilayer structures with controlled thickness. MOCVD (Metal Organic Chemical Vapor Deposition), also called as MOVPE (Metal Organic Vapor Phase Epitaxy) has proven to be extremely important for manufacturing optoelectronics since the past few decades. Before that, LPE (Liquid Phase Epitaxy), VPE (Vapor Phase Epitaxy) and MBE (Molecular Beam Epitaxy) were used [19] [29].

All group III-N materials used in this study were grown in an Aixtron 200/4 RF-S MOVPE system shown in Figure 2.5. MOVPE is a complex growth technique which takes places in gas phase and involves surface chemical reactions. Metal-organic precursors are used as source materials for the growth of IIINitrides. Trimethylgallium (TMGa), triethylgallium (TEGa), trimethylaluminum(TMAl) and trimethylindium (TMIn) are available for GaN based alloy thin films growth. Monosilane (SiH4) and biscyclopentadienyl magnesium (CpzMgj) are used for n-type and p-type dopings respectively. Nitrogen and hydrogen are used as carrier gases to transport the MO molecules to the growth site [30]. 


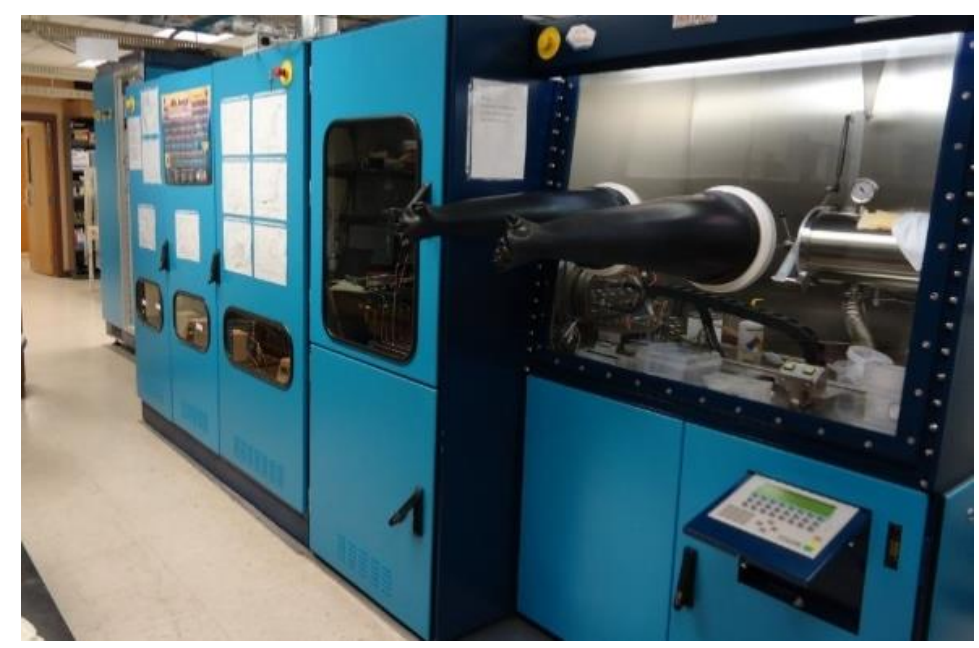

Figure 2.5: Picture of the Aixtron 200/RF-S MOVPE system used for the growth of all III-nitride materials used in this work.

\subsection{Applications}

Group III-V semiconductor materials possess several properties making them attractive in the development of optoelectronic devices for a broad range of applications. Major applications include light emitting diodes (LEDs), Lasers and Photodiodes (PDs) [31]. Figure 2.5 shows a typical configuration of a quantum well LED grown on a sapphire substrate and the close interplay between numerous research groups of advanced III-V LEDs.

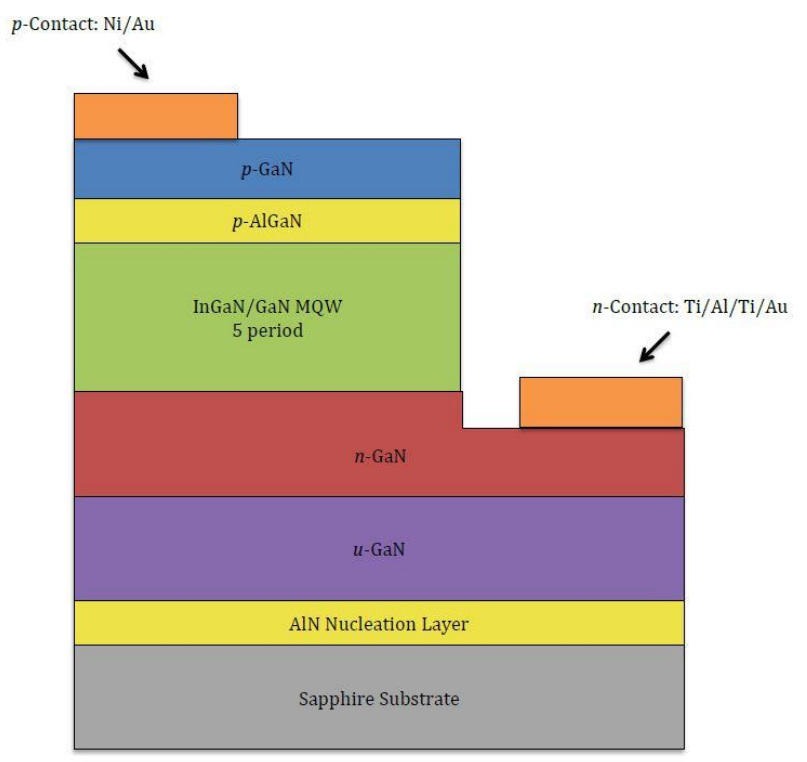




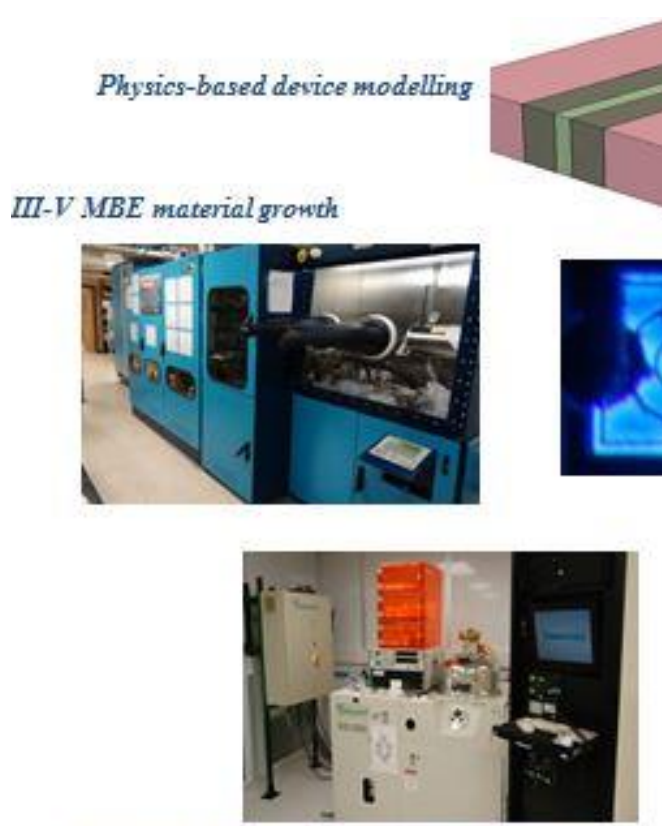

High resolution electron beam lithography
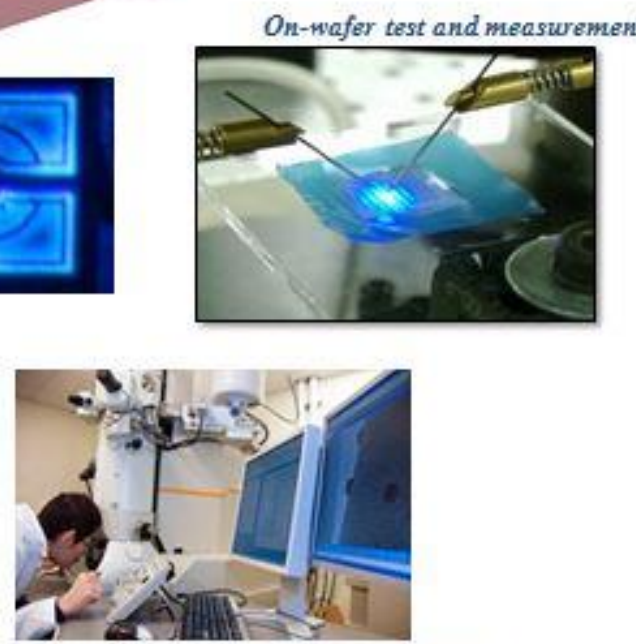

Structural Analysis

(b)

Figure 2.6: (a) Cross section of an III-V LED device (b) advanced III-V LEDs.

In addition to optoelectronic applications, nitride materials also play a crucial role in communication technology. Due to its extraordinary electron transport properties and high injection velocities, High electron mobility transistors (HEMTs) attract more attention. Figure 2.6 shows an advanced III-V HEMT.

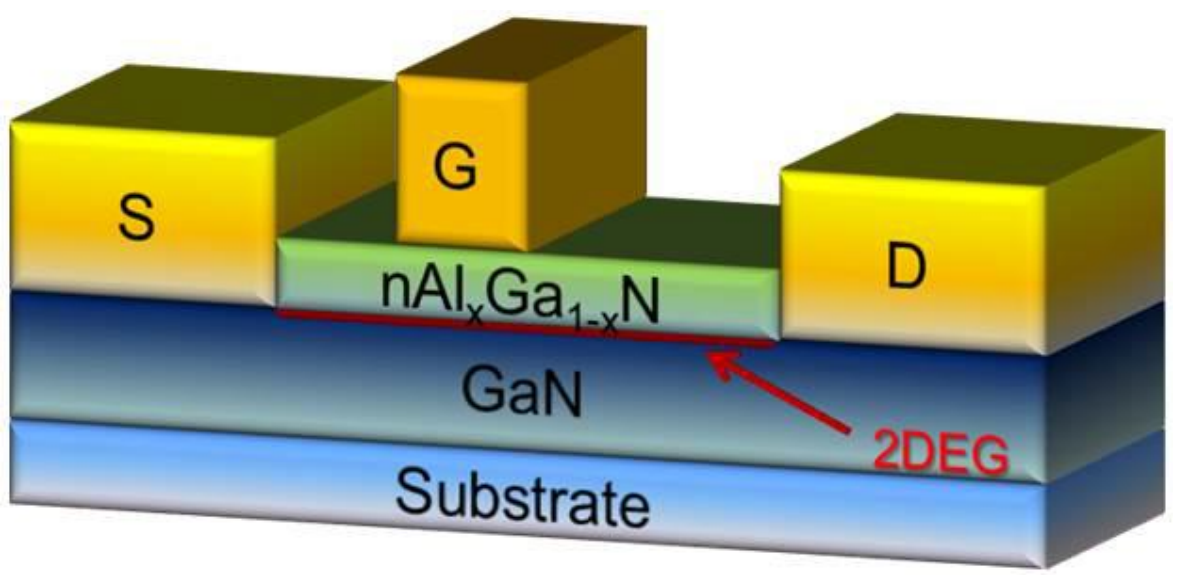

Figure 2.7: Schematic of HEMT epitaxial structure with location of the 2D electron gas shown as a red. 


\section{Chapter 3 Semiconductor Physics}

This chapter presents a discussion of quantum well physics and quantum well structures in light emitting diodes. A related phenomenon - particle in a box model is reviewed in the physics section. It is followed by the discussion of physical effects of quantum wells on light emitting diode characteristics.

\subsection{Quantum Mechanics}

Quantum well is a potential well which is formed in a material due to variation of chemical composition. It confines particles (typically electrons or holes) in a desired region of space and controls quantum effects. The quantum confinement effects become considerable as the quantum well thickness approaches the de Broglie wavelength of the particles. The quantum effects are characterized through energy levels called "energy sub-bands" [32].

In 1923, Louis de Broglie made a proposal that electrons might exhibit wave properties. He proposed that all material objects have a wave nature. The de Broglie wavelength is given by the expression [33]:

$$
\lambda=\frac{h}{p}
$$

or

$$
\lambda=\frac{h}{m v}
$$

where, $\lambda$ is wavelength, $p$ is the momentum, $m$ is the mass, $v$ is the speed of the object and $h$ is Planck's constant.

\subsubsection{Schrodinger Equation}

An important approach for describing quantum mechanical behavior is the Schrodinger wave theory. The Schrodinger wave equation is a partial differential equation in terms of wave function which predicts the probability of events or outcome [34] [35]. 
The time independent Schrodinger equation is given by [34]

$$
\begin{aligned}
\frac{-\hbar^{2}}{2 m_{0}} \nabla^{2} \psi(r, t)+V(r, t) \psi(r, t) & =i \hbar \frac{\partial}{\partial t} \psi(r, t) \\
& =E \psi(r, t)
\end{aligned}
$$

where,

$$
\begin{aligned}
& \hbar \text { is the reduced Planck Constant where } \hbar=\frac{h}{2 \pi} \\
& \qquad m_{0} \text { is mass of an electron } \\
& \psi(r, t) \text { is the wave function } \\
& V(r, t) \text { is the potential energy as a function of position }
\end{aligned}
$$

$E$ is the energy, a real number

$\partial / \partial t$ indicates a partial derivative

$$
\nabla^{2} \text { is the Laplacian }
$$

The above equation can be separated into a time dependent part and a time independent part when $U(r, t)$ has no time dependence. The time independent Schrodinger equation becomes [34]

$$
\left[\frac{-\hbar^{2}}{2 m_{0}} \nabla^{2}+V(r)\right] \psi(r)=E \psi(r)
$$

and the time dependent equation becomes [34]

$$
i \hbar \frac{\partial f(t)}{\partial t}=E f(t)
$$

The general form of time dependent equation can be expressed as [34]

$$
f(t)=e^{ \pm i E t / \hbar}
$$

An application of Schrodinger equation is the particle in a box model which explains particle confinement in an infinite well [35] 


\subsubsection{Particle in a Box}

Particle in a box model is an important illustrative problem used to describe the quantum well physics. It is a fundamental quantum mechanical approach that describes the translational motion of a particle confined in an infinite well surrounded by impenetrable barriers. Schrodinger wave equation is used to solve the problem. The solutions of the equation give possible values of $\mathrm{E}$, allowed energy values and $\psi$, wave functions in the quantum well and also the probability of finding a particle in a region of space at a particular energy level can be estimated [36].

\subsubsection{One dimensional Solution}

A one dimensional system is the simplest form of the particle in a box model. Figure 3.1 represents an infinite quantum well with zero potential energy inside the well and infinite outside the well.

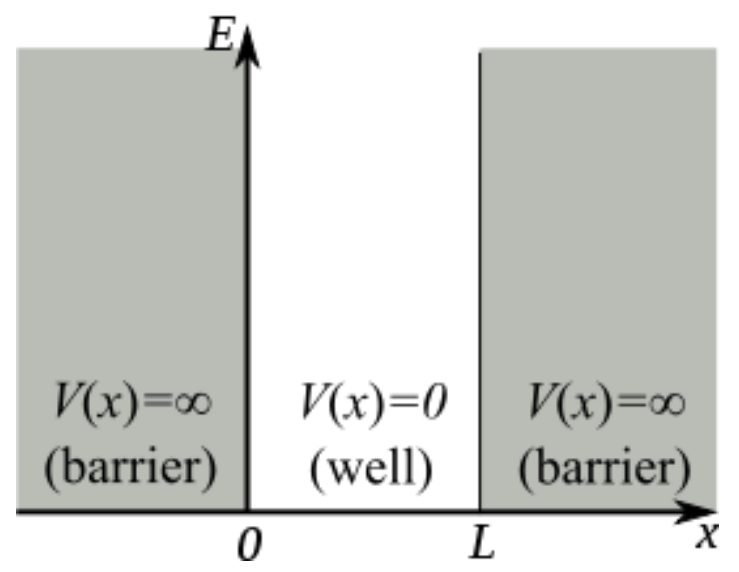

Figure 3.1: Infinite Potential Well (Benjamin D. Esham [37])

The potential energy profile for an infinite quantum well is given by,

$$
\begin{array}{r}
V(x)=0,0<x<L \\
=\infty, \text { otherwise }
\end{array}
$$

where, $L$ is the length of the well and $x$ is the position of the particle within the well.

The time independent Schrodinger equation (3.2) for this one dimensional system is given as,

$$
-\frac{\hbar^{2}}{2 m} \frac{d^{2} \psi(x)}{d x^{2}}+V(x) \psi(x)=E \psi(x)
$$


Equation (3.6) can be modified for a particle of mass $m$ moving inside the well with zero potential energy resulting in the quantum mechanical description in one dimension,

$$
-\frac{\hbar^{2}}{2 m} \frac{d^{2} \psi(x)}{d x^{2}}=E \psi(x)
$$

The general solution to equation (3.7) can be given as [38],

$$
\psi(x)=A \sin (k x)+B \cos (k x)
$$

where $A, B$ and $k$ are constants.

Solving for the constants results in [38],

$$
\begin{gathered}
A=\sqrt{\frac{2}{L}} \\
k=\left(\frac{8 \pi^{2} m E}{h^{2}}\right)^{1 / 2}
\end{gathered}
$$

By solving equation (3.11) using the boundary conditions, the normalized wave function for a particle in a 1-dimensional box can be obtained as shown in equation (3.12) [38],

$$
\begin{gathered}
\int_{0}^{L} \psi^{2} d x=1 \\
\psi=\sqrt{\frac{2}{L}} \sin \frac{n \pi}{L} x
\end{gathered}
$$

where, $\mathrm{n}$ is a positive integer representing wave number.

Solving for E results in the allowed energies for a particle in a box [38],

$$
E_{n}=\frac{n^{2} h^{2}}{8 m L^{2}}
$$

A sketch of these wave functions, energy levels and probability density functions in an infinite square quantum well can be seen in Figure 3.2. 


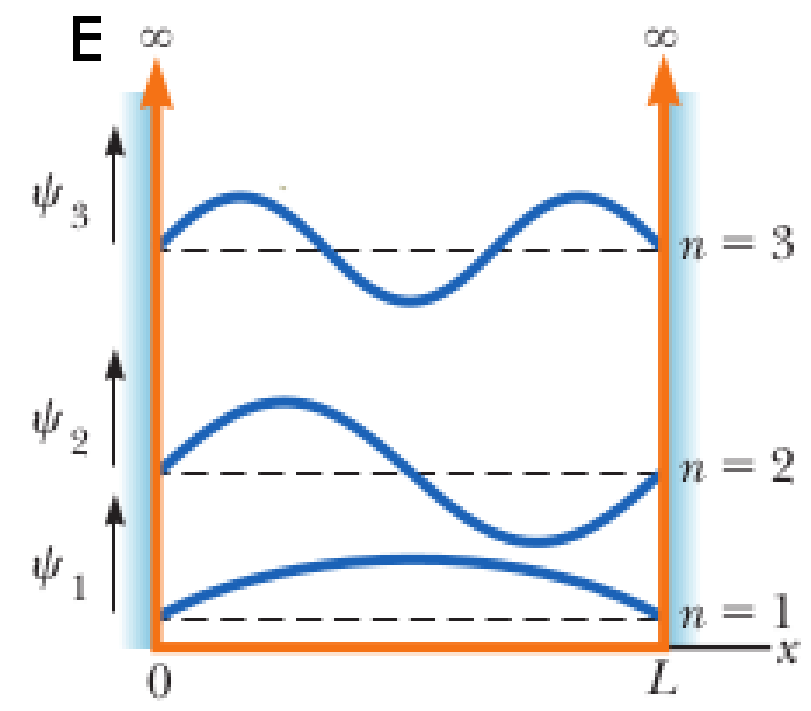

(a)

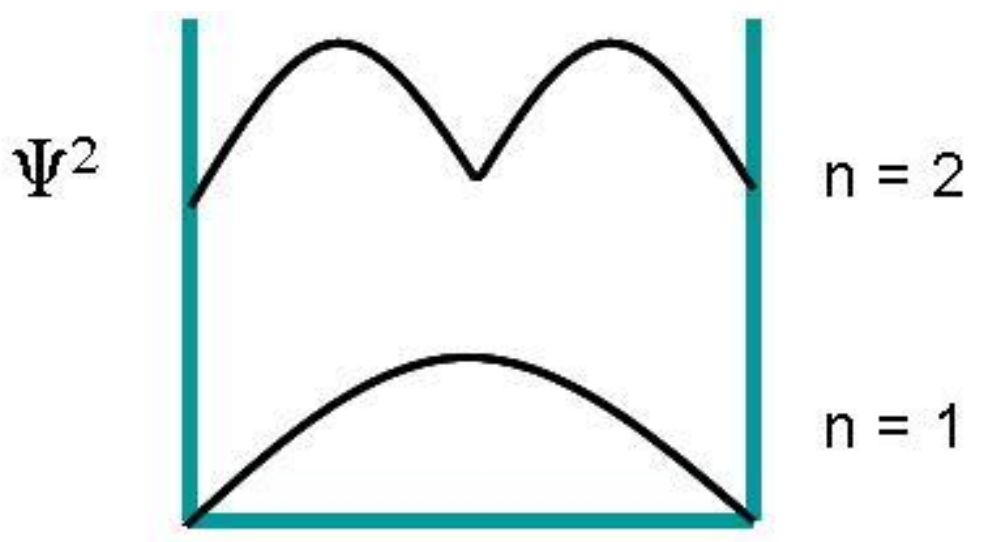

(b)

Figure 3.2: (a) Wave functions and energy levels in an infinite square potential well $(R . A$. Serway, J. John W. Jewett [39]) (b) Probability distribution of a particle in a box (Prof. Delmar Larsen [36])

\subsubsection{Higher dimensional boxes}

If a quantum particle of mass $\mathrm{m}$ is trapped in a two-dimensional rectangular box, it may freely move in the $x$ and $y$-directions, between barriers separated by lengths $L_{x}$ and $L_{y}$ respectively. The wave functions and energies can be obtained by solving the Schrodinger equation using a similar approach to that of the one-dimensional box. Thus, the wave function $\psi(x, y)$ is of the form [38], 


$$
\psi_{n_{x} n_{y}}=\sqrt{\frac{4}{L_{x} L_{y}}} \sin \left(\frac{n_{x} \pi x}{L_{x}}\right) \sin \left(\frac{n_{y} \pi y}{L_{y}}\right)
$$

The allowed values of the total energy are given by [38],

$$
E_{n_{x} n_{y}}=\frac{\hbar^{2} \pi^{2}}{2 m}\left(\frac{n_{x}^{2}}{L_{x}{ }^{2}}+\frac{n_{y}^{2}}{L_{y}^{2}}\right)
$$

For a three dimensional box, the wave functions become [40]

$$
\psi_{n_{x} n_{y} n_{z}}=\sqrt{\frac{8}{L_{x} L_{y} L_{z}}} \sin \left(\frac{n_{x} \pi x}{L_{x}}\right) \sin \left(\frac{n_{y} \pi y}{L_{y}}\right) \sin \left(\frac{n_{z} \pi z}{L_{z}}\right)
$$

and the allowed values of the total energy are [40]

$$
E_{n_{x} n_{y} n_{z}}=\frac{\hbar^{2} \pi^{2}}{2 m}\left(\frac{n_{x}^{2}}{L_{x}{ }^{2}}+\frac{n_{y}^{2}}{L_{y}{ }^{2}}+\frac{n_{z}^{2}}{L_{z}{ }^{2}}\right)
$$

\subsubsection{Finite Potential Well}

The infinite potential well is a valuable model that explains the concept of quantum mechanics. However, the degree of focus here is quantum wells in the semiconductor devices. A quantum well is a potential well, in which a particle is confined to a finite depth box. Unlike the infinite potential well, there is a probability associated with the particle being found outside the box. A simple model of the energy levels in a quantum well is considered below. Consider a potential well centered on the origin of width $L$ and barrier height $U$ as shown in Figure 3.3.

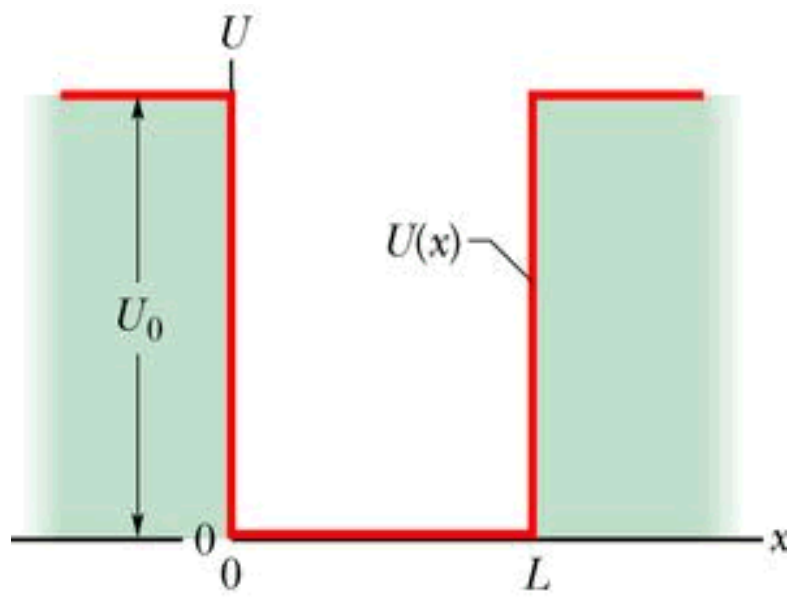

Figure 3.3: Finite Potential Well (chegg.com [41]) 
The potential profile is given as,

$$
U(x)=\left\{\begin{array}{l}
U, x \leq 0(\text { region } I) \text { and } x \geq L(\text { region } I I I) \\
0,0<x<L(\text { region } I I)
\end{array}\right.
$$

Outside the box:

Since the potential is constant, the Schrodinger time-independent equation (3.8) reduces to

$$
\frac{\hbar^{2}}{2 m} \frac{d^{2} \psi(x)}{d x^{2}}+(E-U) \psi(x)=0, x \leq 0
$$

and

$$
\frac{\hbar^{2}}{2 m} \frac{d^{2} \psi(x)}{d x^{2}}+(E-U) \psi(x)=0, x \geq L
$$

The general solution becomes as [42],

$$
\psi(x)=A e^{k x}+B e^{-k x}
$$

Wavefunction $\psi(x)$,

$$
\begin{gathered}
\psi_{I}(x)=A e^{k x} \\
\psi_{I I I}(x)=B e^{-k x}
\end{gathered}
$$

Require continuity of wavefunctions at $x=0$ and $x=L$.

Inside the box:

Schrodinger equation (3.8) reduces to

$$
\frac{\hbar^{2}}{2 m} \frac{d^{2} \psi(x)}{d x^{2}}+E \psi(x)=0,0<x<L
$$

The general solution is given as [42],

$$
\psi(x)=C \cos k x+D \sin k x
$$

Hence,

$$
E=\frac{k^{2} \hbar^{2}}{2 m}
$$


Figure 3.4 shows the wave functions of a finite potential well.

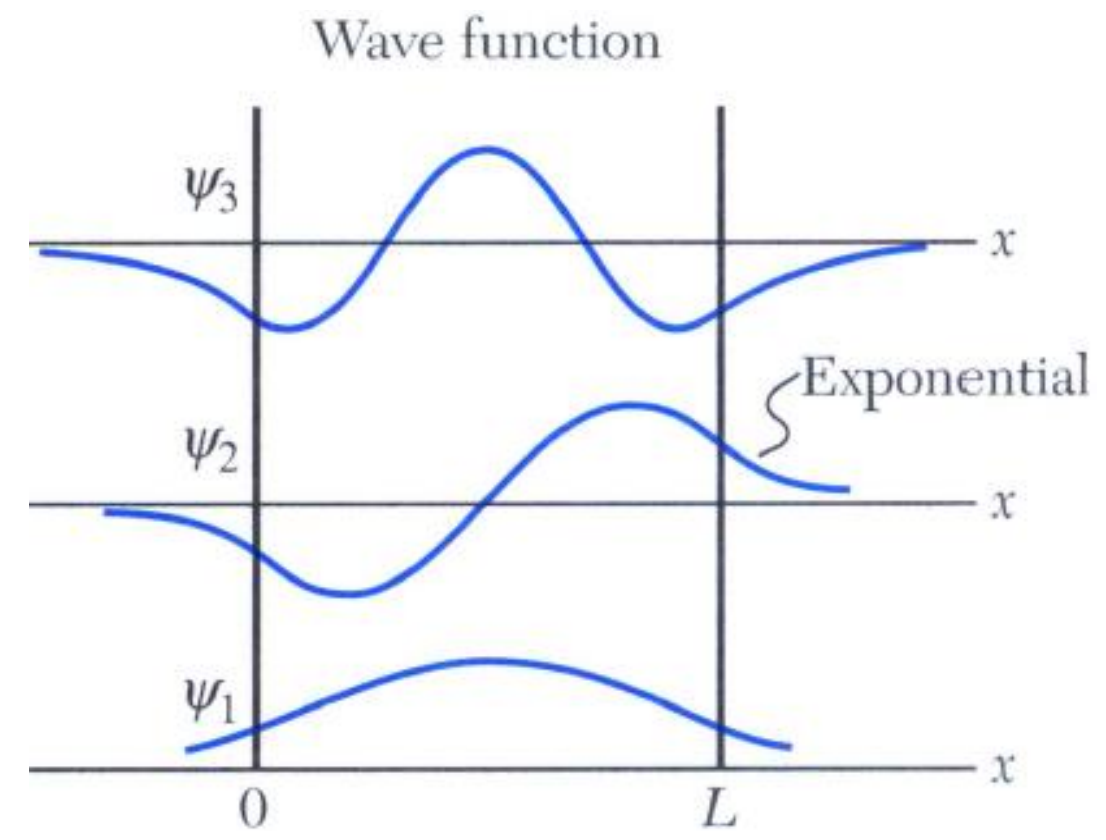

Figure 3.4: Wave Functions (L. Hrommhold [42])

\subsection{Quantum Well Structure}

A quantum well is a thin layer of a semiconductor material sandwiched between two layers of a material with a wider bandgap. Such thin layers can be grown by molecular beam epitaxy (MBE) or metal-organic chemical vapor deposition (MOCVD) [43]. In general, materials that are used to form quantum well structures belong to group III-V. Few examples include GaAs QW embedded in AlAs, InGaN in GaN, GaAs in AlGaAs and InGaAs in GaAs. Figure 3.5 shows a quantum well and its band structure.

Embedding quantum well layers into semiconductor devices results in better performance. Due to its small thickness, quantum wells allow the use of quantum mechanics as an engineering tool. One can modify electron and hole wavefunctions by controlling the width of the quantum wells. These quantum well structures permit advancements in optical applications leading to new optical modulators and switches. Quantum wells can also be used to make electronic devices, including high-speed filed effect transistors and resonant tunneling structures [44]. 


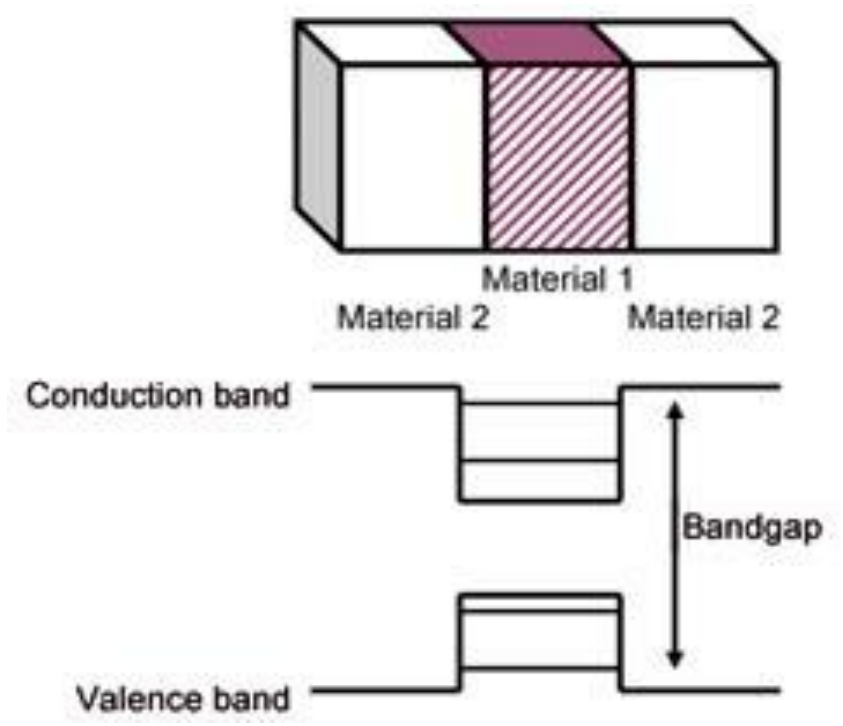

Figure 3.5: Quantum Well Structure (IRnova [45])

Structures containing more than one quantum well layer are known as multiple quantum well devices. Multiple quantum wells improve the overall performance of the devices. MQW devices have many of the spectral characteristics of SQW devices. One main reason for using the MQW structure is the possibility of reduced carrier densities. A decrease in active-layer carrier density is central to improving the high-current efficiency of InGaN-based light emitters [46]. Figure 3.6 shows a multiple quantum well LED.
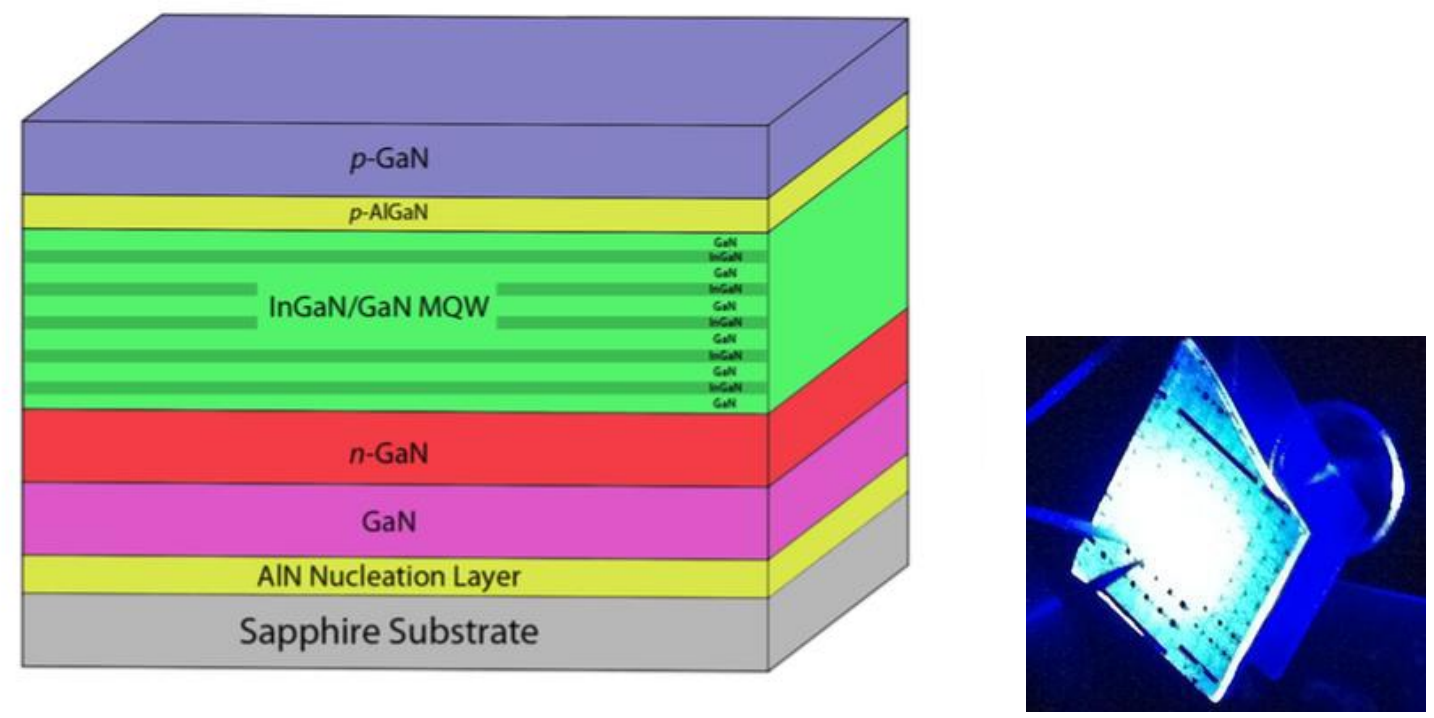

Figure 3.6: (a) Multiple Quantum Well Blue LED(b) WVU Fabricated LED 


\subsection{Light Emitting Diode}

A light emitting diode (LED) is a basic semiconductor p-n junction diode that emits light. When operated in a forward bias direction, the current flows from the $p$-side to the $n$-side. This results in an electron-hole pair recombination within the device, thus releasing energy in the form of photons. This effect is called electroluminescence, and the wavelength of the light is determined primarily by the energy band gap of the materials forming the $p-n$ junction using equation (3.29). The materials used for the LED have a direct band gap with energies corresponding to near-infrared, visible, or near-ultraviolet light [47]. Figure 3.7 shows circuit and band diagram of a LED and Figure 3.8 shows an electromagnetic spectrum as a function of wavelength. Energy of the emitted photon,

$$
E_{g}=\frac{h c}{\lambda}
$$

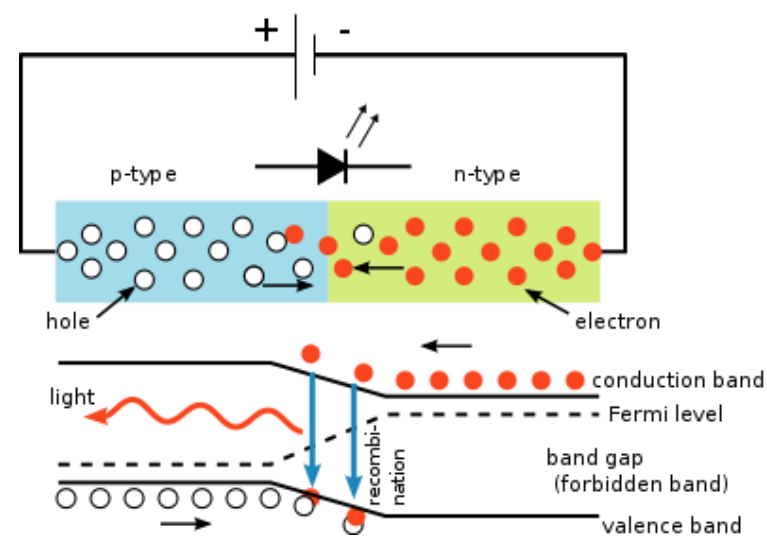

Figure 3.7: LED circuit and band diagram (S-kei [48])

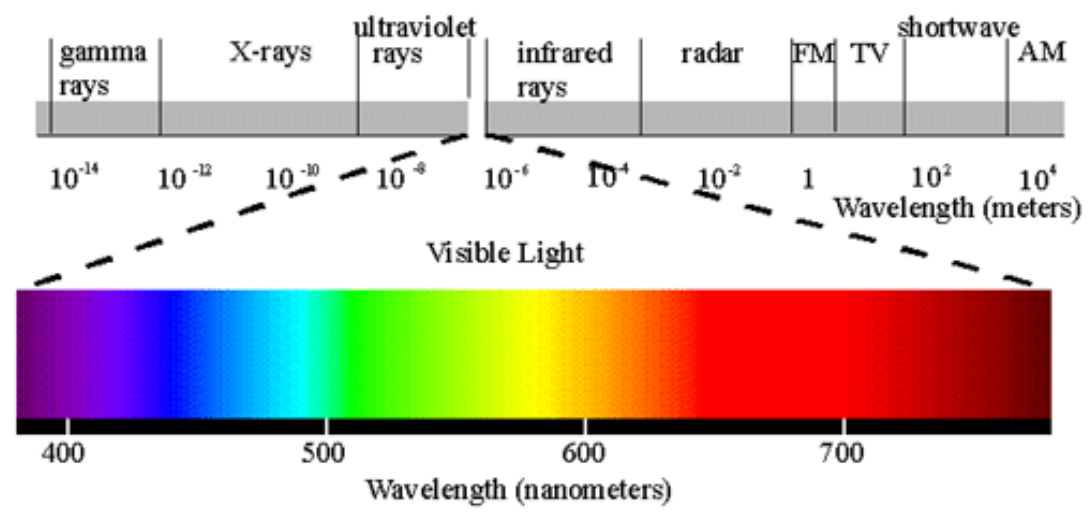

Figure 3.8: Electromagnetic Spectrum (Aladdin LED Lights \& Lamps [49]) 
The Light Emitting Diode characteristic Electro Luminescence Spectra was studied in this thesis and is presented below with an in detail explanation.

\subsubsection{Spontaneous Emission}

The two distinct emission processes by which the emission of a photon can occur in optoelectronic devices are spontaneous emission and stimulated emission. In these fundamental processes, atoms are excited from ground state to final state there by resulting in the emission of light. Spontaneous emission occurs in light emitting diodes whereas stimulated emission is employed in lasers. The process of spontaneous emission is illustrated through an energy band diagram as shown in Figure 3.9.
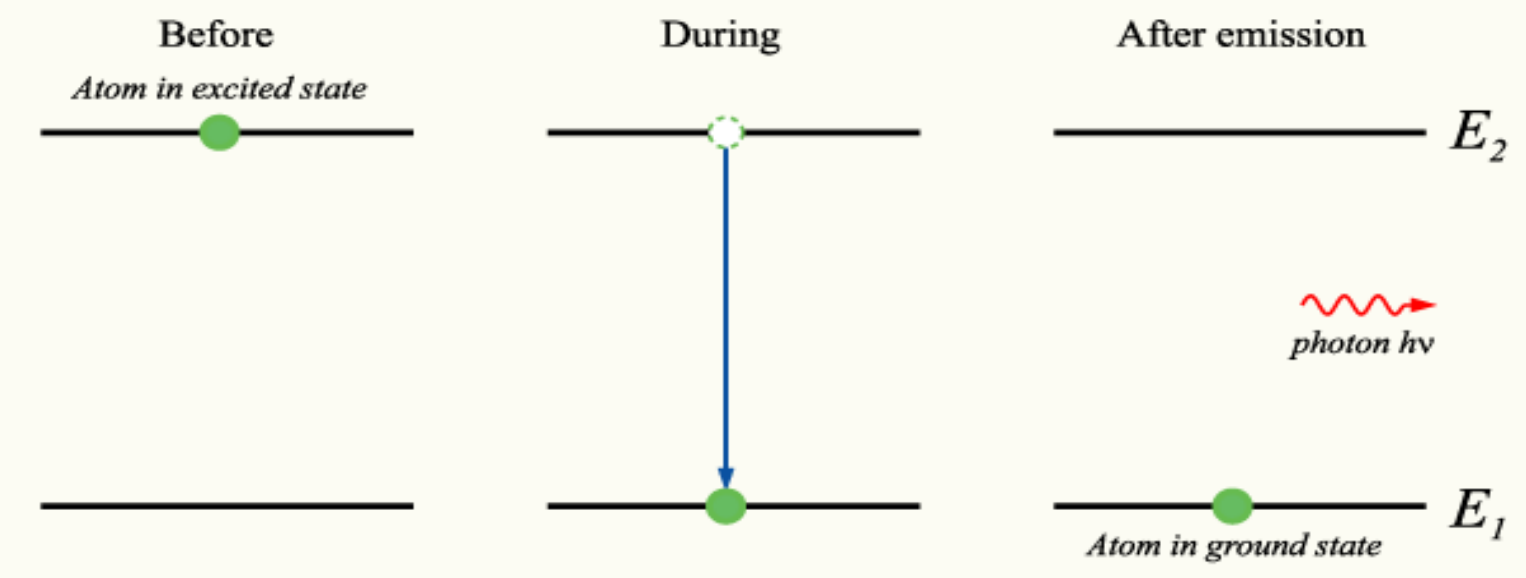

Figure 3.9: Spontaneous Emission (IImari Karonen [50])

The electron-hole recombination process follows the Boltzmann distribution and the emission intensity is expressed in terms of energy as follows [11]:

$$
I(E) \alpha \sqrt{E-E_{g}} e^{-E /(k T)}
$$

Figure 3.9 gives the line shape of the LED from equation (3.30). The maximum emission intensity occurs at

$$
E=E_{g}+\frac{1}{2} k T
$$

and the full width half maximum (FWHM) of the emission line is 1.8kT [11]. 


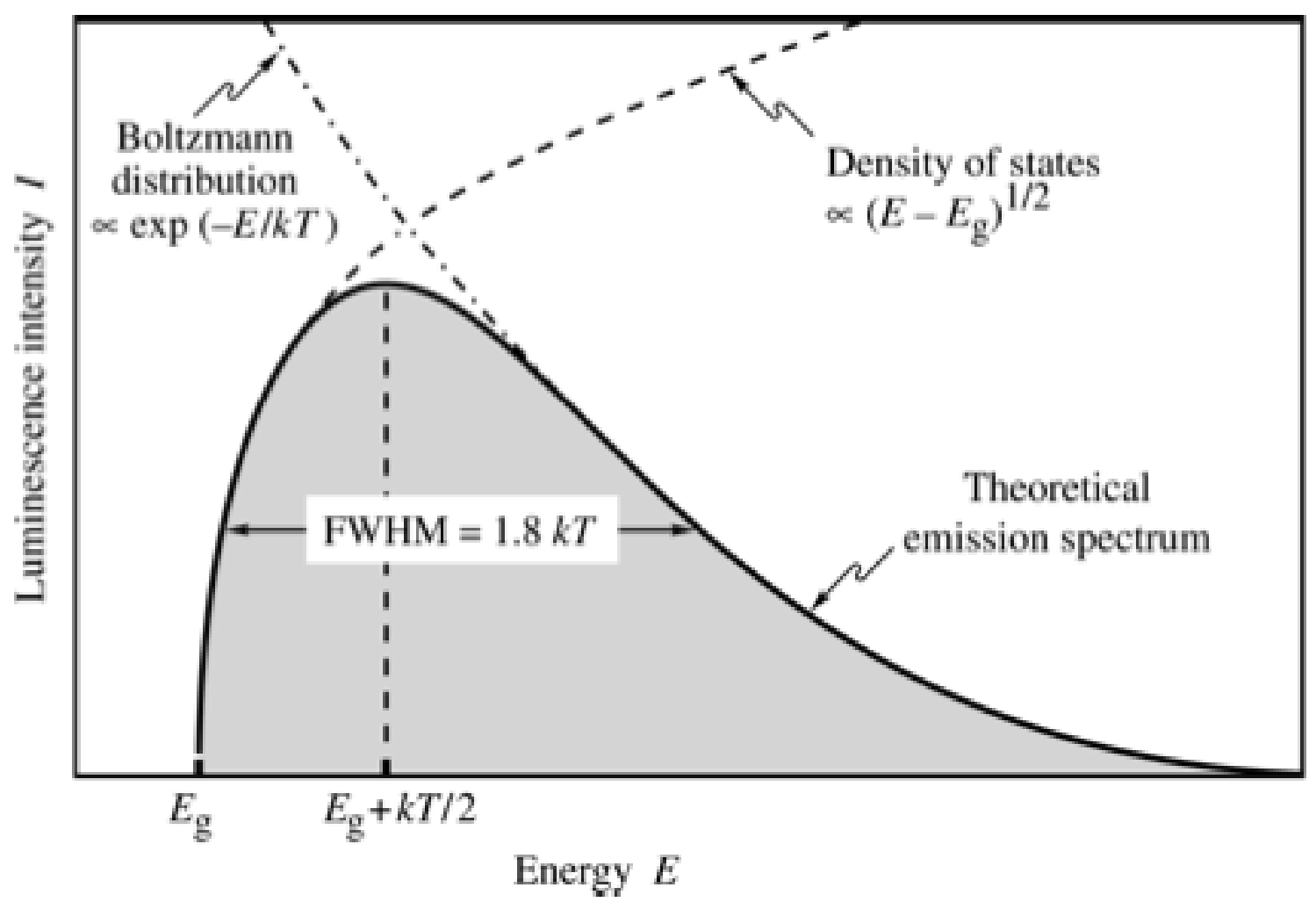

Figure 3.10: Theoretical emission spectrum of an LED (E. Fred Schubert [11]) 


\section{Chapter 4 TCAD Tools and Physical Models}

This chapter gives an overview of the simulation tools and the physical models used for the comprehensive simulation of semiconductor multiple quantum well optoelectronic devices with an in detail explanation. The simulation templates used for this study are Synopsys TCAD Sentaurus Workbench Version H-2013.03, tiberCAD 2.1.0 and Crosslight APSYS.

\subsection{Synopsys Sentaurus}

Sentaurus is a suite of Technology Computer-Aided Design (TCAD) tools developed by Synopsys which are used for developing and optimizing the semiconductor processing technologies and devices. The Sentaurus simulators solve physical models allowing an exploration of structural properties and electrical behavior of semiconductor devices. Sentaurus TCAD supports a broad range of applications covering silicon and compound semiconductor technologies. The tools which have been used in this thesis include [51] [52]:

- Sentaurus Workbench (SWB): A graphical interface designed to create, manage, execute and analyze sequential simulations right from the structure creation to device operation by integrating all the other tools into a single entity. It allows users to navigate between multiple simulations performed on a single device such as preprocessing of user input files, parameterizing the structural and physical properties and visualizing results. Sentaurus Workbench automatically creates the output file of one tool and then, after its successful completion, passes the output files to next tool in the queue removing the need to control it manually by the user [52] [53].

- Sentaurus Structure Editor (SSE): Sentaurus Structure Editor is a two-dimensional (2D) and threedimensional (3D) device editor developed to design device structures before simulation. It can perform simulations through a graphical user interface (GUI) or by using an input file based on Scheme scripting language. It provides an inherent flexibility allowing the user to define any physical property or dimension of the device as a variable which can be changed to perform a series of experiments for different values of a parameter. It divides the structure into a series of discrete points on which physical equations will be solved by creating a mesh. The output file created by this tool contains the geometrical, material, contact and doping information about the device. It is this file that is used by other tools to perform device simulations [52] [54] [55]. 
- Sentaurus Device (SD): Sentaurus Device is a multidimensional simulator for semiconductor devices. It incorporates an extensive set of physical models, material parameters and robust numeric methods. It is used to simulate electrical, thermal and optical characteristics of compound semiconductor devices by solving a set of diffusion and transport equations numerically based on physical parameters and boundary conditions. These simulations generate output files containing information on carrier densities, fields, potentials, energy levels and temperatures inside the device at various point of time. In addition, Sentaurus Device also enables complex integrated circuit phenomena and models for simulating state-of-the-art logic and memory devices [52] [54] [55].

- SVisual and Inspect: A set of visualization tools to observe the 3D and 2D variations respectively, both in the device structure and during simulation. The Synopsys Sentaurus Visual tool is a plotting software for visualizing data from simulations and experiments. It allows users to visualize complex simulation results in one, two and three dimensions. It provides the flexibility to work in both graphical user interface and scripting language environment. It can also be used to create plots, extract data and rotate images [56]. Inspect can be used to display and analyze curves. It features a graphical user interface (GUI), a script language, and an interactive language for computations with curves. An Inspect curve is a sequence of points defined by an array of $x$-coordinates and $y$ coordinates. An array of coordinates that can be mapped to one of the axes is referred to as a dataset. With Inspect, datasets can be combined and mapped to the $x$-axis and $y$-axis to create and display a curve [57].

In the Sentaurus TCAD software, a number of physical models are provided to describe the device physics as closely as possible to the real device. These models deal with the behavior of the carrier in combined effects of various boundary conditions like lattice temperature, electrostatic potentials and fields, external forces, hetero-structures band gap variations and quantum effects. Such models, depending on the type of device and operating conditions, have to be included in the model to perform simulations and do reliable predictions about the device characteristics. One of the focuses of this study was to study optical characteristics of Light Emitting Diodes and photoluminescence spectra of quantum wells using Synopsys. Reference has been given to refer for information about physical models used for electrical optimization of Light Emitting Diodes [58]. The physical models implemented in this study were:

- Optical Generation

- Quantum Yield Models 
- Optical Solver

- Complex Refractive Index Model

\subsubsection{Optical Generation}

The generation rate gives the number of electrons generated at each point in the device due to the absorption of photons. Neglecting reflection, the amount of light which is absorbed by a material depends on the absorption coefficient $\left(\alpha\right.$ in $\mathrm{cm}^{-1}$ ) and the thickness of the absorbing material. The intensity of light at any point in the device can be calculated according to the equation [59]:

$$
I=I_{0} e^{-\alpha x}
$$

where $\alpha$ is the absorption coefficient typically $\mathrm{cm}^{-1}$;

$x$ is the distance into the material at which the light intensity is being calculated; and

$I_{0}$ is the light intensity at the top surface.

Consequently, differentiating the above equation will give the generation at any point in the device. Hence [59]:

$$
G=\alpha N_{0} e^{-\alpha x}
$$

where $N_{0}$ is the photon flux at the surface (photons/unit-area/sec).

The absorption of light in a semiconductor causes the optical power to decrease with distance. The optical generation rate profile i.e. Figure 4.1 shows that the optical generation rate decreases as a function of depth. 


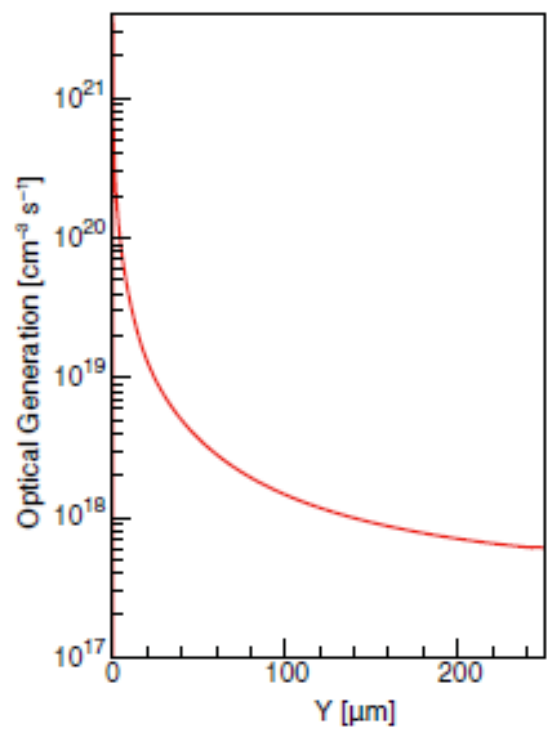

Figure 4.1: Optical generation rate as a function of depth (Synopsys [59])

Sentaurus Device supports various methods that are used to compute optical generation rate when an optical wave penetrates into the device, is absorbed, and produces electron-hole pair. The optical generation resulting from a monochromatic optical source or an illumination spectrum is determined as the product of the absorbed photon density, which is computed by the optical solver, and the quantum yield.

\subsubsection{Quantum Yield Models}

The quantum yield is defined as the ratio of number of photons emitted to the number of photons absorbed and is given as [60]:

$$
\emptyset=\frac{\text { number of photons emitted }}{\text { number of photons absorbed }}
$$

The quantum yield model describes how many of the absorbed photons are converted to generated electron-hole pairs. The simplest model assumes that all absorbed photons result in generated charge carriers irrespective of the band gap or other properties of the underlying material. This corresponds to a global quantum yield factor of one, which is the default value, except for nonsemiconductor regions where it is always set to zero even if photons are actually absorbed. More realistic models take the band gap into account [54]. 


\subsubsection{Optical Solver}

Several optical solvers are available to compute the optical generation and the choice for a specific method is determined usually by the optimum combination of accuracy of results and computation time. The following optical solvers are used in this study:

- Transfer matrix method (TMM)

- $\quad$ Raytracing (RT)

\subsubsection{Transfer Matrix Method}

The transfer-matrix method is a method used in optics to calculate the propagation of plane waves through layered media. When a plane wave is incident on a layered medium, it undergoes reflections and transmissions at the layer boundaries. These reflections in turn result in their own reflections and transmissions in an unending process. Fresnel equations are used to determine the complex amplitudes of these transmitted and reflected waves which are facilitated by the use of matrix methods. The matrix $M$, is called the transfer matrix and depends on the optical properties of the layered medium [61].

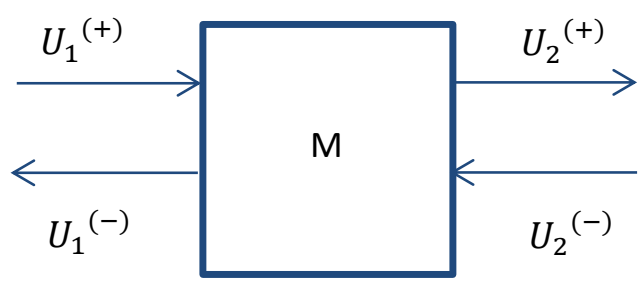

(a) Single Layered Medium

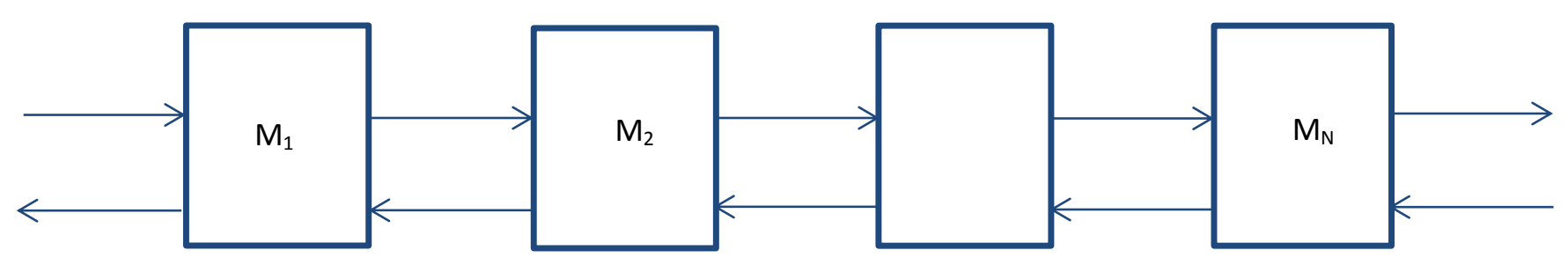

(b) Multilayered Medium

Figure 4.2: Wave amplitudes in a layered medium and transfer matrices connecting them (B. E. A. Saleh, M. C. Teich [61]) 
The transfer matrix for the single layered medium is defined by [54]:

$$
\left[\begin{array}{l}
U_{2}{ }^{(+)} \\
U_{2}{ }^{(-)}
\end{array}\right]=\left[\begin{array}{ll}
A & B \\
C & D
\end{array}\right]\left[\begin{array}{l}
U_{1}{ }^{(+)} \\
U_{1}{ }^{(-)}
\end{array}\right]
$$

where, $U_{1}{ }^{(+)}$and $U_{1}{ }^{(-)}$are amplitudes of the forward and backward collected waves at plane 1, $U_{2}{ }^{(+)}$and $U_{2}{ }^{(-)}$are amplitudes of the forward and backward collected waves at plane 2 and $A, B, C$ and $D$ are elements of matrix $M$.

For a multilayered medium, the matrix is defined by [61]:

$$
M=M_{N} \ldots M_{2} M_{1}
$$

\subsubsection{Ray Tracing}

In Sentaurus Device, the raytracer has been implemented based on linear polarization. It is optimized for speed and needs to be used in conjunction with the complex refractive index model. The raytracer uses a recursive algorithm: It starts with a source ray and builds a binary tree that tracks the transmission and reflection of the ray. A reflection/transmission process occurs at interfaces with refractive index differences [54].

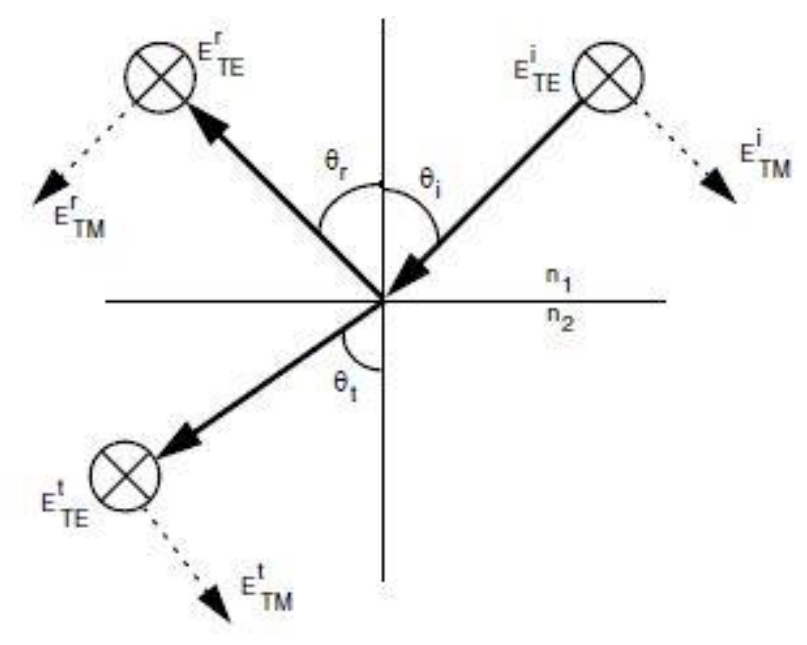

Figure 4.3: Reflection/Transmission Process (Synopsys [54])

An incident ray impinges on the interface of two different refractive index $\left(n_{1}\right.$ and $\left.n_{2}\right)$ regions, resulting in a reflected ray and a transmitted ray. The incident, reflected, and transmitted rays are denoted by the subscripts $i, r$, and $t$, respectively. Likewise, the incident, reflected, and transmitted 
angles are denoted by $\theta_{i}, \theta_{r}$, and $\theta_{t}$, respectively. These angles can be derived from the concept of interface tangential phase-matching (commonly called Snell's law) using [54]:

$$
n_{1} \sin \theta_{i}=n_{2} \sin \theta_{t}
$$

A ray can be considered a plane wave traveling in a particular direction with its polarization vector perpendicular to the direction of propagation. The length of the polarization vector represents the amplitude, and the square of its length denotes the intensity. The TE polarization (s-wave) applies to the ray polarization vector component that is perpendicular to the plane of incidence. On the other hand, the TM polarization ( $p$-wave) applies to the ray polarization vector component that is parallel to the plane of incidence. The TE and TM components of the ray polarization vector are denoted in Figure 4.3.

The raytracer automatically computes the plane of incidence at each interface, decomposes the polarization vector into TE and TM components, and applies the respective reflection and transmission coefficients to these TE and TM components [54].

\subsubsection{Complex Refractive Index Model}

The complex refractive index model in Sentaurus Device allows you to define the refractive index and the extinction coefficient depending on mole fraction, wavelength, temperature, carrier density, and local material gain. In addition, it provides a flexible interface that can be used to add new complex refractive index models as a function of almost any internally available variable [54].

The complex refractive index $\tilde{n}$ can be written as [54]:

$$
\tilde{n}=n+i . k
$$

with:

$$
\begin{gathered}
n=n_{0}+\Delta n_{\lambda}+\Delta n_{T}+\Delta n_{\text {carr }}+\Delta n_{\text {gain }} \\
k=k_{0}+\Delta k_{\lambda}+\Delta k_{\text {carr }}
\end{gathered}
$$

The real part $n$ is composed of the base refractive index $n_{0}$, and the correction terms $\Delta n_{\lambda}, \Delta n_{T}, \Delta n_{\text {carr }}$, and $\Delta n_{\text {gain }}$. The correction terms include the dependency on wavelength, temperature, carrier density and gain. The imaginary part $k$ is composed of the base extinction coefficient $k_{0}$, and the correction 
terms $\Delta k_{\lambda}$ and $\Delta k_{\text {carr }}$. The correction terms include the dependency on wavelength and carrier density. The absorption coefficient $\alpha$ is computed from $k$ and wavelength $\lambda$ according to [54]:

$$
\alpha=\frac{4 \pi}{\lambda} \cdot k
$$

\subsubsection{Wavelength Dependency}

The complex refractive index model offers three ways to take wavelength dependency into account:

- An analytic formula considers a linear and square dependency on the wavelength $\lambda$ [54]:

$$
\begin{aligned}
& \Delta n_{\lambda}=C_{n, \lambda} \cdot \lambda+D_{n, \lambda} \cdot \lambda^{2} \\
& \Delta k_{\lambda}=C_{k, \lambda} \cdot \lambda+D_{k, \lambda} \cdot \lambda^{2}
\end{aligned}
$$

- Tabulated values can be read from the parameter files.

- Tabulated values can be read from the external files.

\subsubsection{Temperature Dependency}

The temperature dependency of the real part of the complex refractive index follows the relation according to [54]:

$$
\Delta n_{T}=n_{0} \cdot C_{n, T} \cdot\left(T-T_{p a r}\right)
$$

\subsubsection{Carrier Dependency}

The change in the real part of the complex refractive index due to free carrier absorption modeled according to [54]:

$$
\Delta n_{\text {carr }}=-C_{n, \text { carr }} \cdot \frac{q^{2} \lambda^{2}}{8 \pi^{2} c^{2} \varepsilon_{0} n_{0}} \cdot\left(\frac{n}{m_{n}}+\frac{p}{m_{p}}\right)
$$

where:

- $C_{n, \text { carr }}$ is a fitting parameter.

- $q$ is the elementary charge.

- $\lambda$ is the wavelength.

- $\quad c$ is the speed of light in free space.

- $\varepsilon_{0}$ is the permittivity.

Furthermore, $n$ and $p$ are the electron and hole densities, and $m_{n}$ and $m_{p}$ are the effective masses of electron and hole. 
A linear model is available to take into account the charge of the extinction coefficient due to free carrier absorption [54]:

$$
\Delta k_{c a r r}=\frac{\lambda}{4 \pi} \cdot\left(C_{k, c a r r, n} \cdot n+C_{k, c a r r, p} \cdot p\right)
$$

The parameters $C_{k, c a r r, n}$ and $C_{k, c a r r, p}$ are fitting parameters.

\subsubsection{Gain Dependency}

The complex refractive index model offers two formulas take into account the gain dependency:

- The linear model is given by [54]:

$$
\Delta n_{\text {gain }}=C_{n, \text { gain }} \cdot\left(\frac{n+p}{2}-N_{\text {par }}\right)
$$

- The logarithmic model reads [54]:

$$
\Delta n_{\text {gain }}=C_{n, \text { gain }} \cdot \ln \left(\frac{n+p}{2 N_{\text {par }}}\right)
$$

\subsubsection{Limitations}

The quantum well transport models and the special meshing required for quantum well region are not well supported by Synopsis Sentaurus as stated by Synopsys Technical Support (March 28, 2014). In the later parts of the study, tiberCAD tool was used to study quantum properties of active region. As Crosslight APSYS tool supports quantum transport and carrier scattering models, it was used to study to analyze characteristics of quantum well LED devices.

\section{2 tiberCAD}

tiberCAD is a multiscale CAD simulation tool used to model and design nanoelectronic and optoelectronic devices such as LEDs, FETs and Dye Solar Cells (DSCs). tiberCAD allows to perform reliable simulations by taking into account the most important physical concepts such as quantum confinement, self-heating, strain and polarization in heterostructures and thermal transport [62]. This tool was used to study quantum properties in confined nanostructures. tiberCAD supports several modules, here are the modules implemented in this study [63]:

- Drift-Diffusion: Calculates poisson-driftdiffusion transport of holes, electrons and excitons. 
- EFA (Envelope Function Approximation): To find quantum properties in quantum well structures.

Each module is explained in detail in the following sections.

\subsubsection{Drift-diffusion}

With tiberCAD, simulation of quantum and classical transport of electrons and holes is based on drift-diffusion approximation. In general, drift current is generated due to charged particle motion in response to an electric field and diffusion current due to distribution of particles from areas of high concentration to areas of lower concentration. The semi-classical model is derived using the following equations [64]:

$$
\begin{aligned}
& J_{n}=q \mu_{n} \mathrm{nE}+\mathrm{q} D_{n} \nabla n \\
& J_{p}=q \mu_{p} \mathrm{pE}-\mathrm{q} D_{p} \nabla p
\end{aligned}
$$

where, $\mathrm{q}$ is the charge of the particle, $\mu$ is the mobility of the semiconductor and measures the ease with which carriers can move through the crystal, $E$ is electric field and D is the diffusion coefficient.

Several options influence the behavior of the Drift-Diffusion module, one such significant module option is coupling. It defines which equations to solve which includes poisson, electron and hole continuity. The continuity equations, which describe charge conservation can be written as [54]:

$$
\begin{aligned}
& \nabla \cdot \overrightarrow{J_{n}}=q R_{n e t}+q \frac{\partial n}{\partial t} \\
& -\nabla \cdot \overrightarrow{J_{p}}=q R_{n e t}+q \frac{\partial p}{\partial t}
\end{aligned}
$$

where:

- $R_{n e t}$ is the net recombination rate

- $\overrightarrow{J_{n}}$ is the electron current density

- $\overrightarrow{J_{p}}$ is the hole current density

- $\mathrm{n}$ and $\mathrm{p}$ are the electron and hole density, respectively. 
The poisson equation is given by [58]:

$$
\nabla \cdot\left(\varepsilon_{0} \varepsilon_{r} \vec{E}\right)=q\left(p-n+N_{D}-N_{A}\right)
$$

where, $N_{D}$ and $N_{A}$ are dopants.

\subsubsection{Polarization Models}

For simulations involving materials with nonzero electric polarization (such as nitrides) it is important to include the effect of polarization. The spontaneous polarization model (sometimes also called 'pyroelectric polarization') imposes a constant electric polarization P along the symmetry-braking direction of the crystal. Crystals with wurtzite structure like Nitrides have strong polarization fields along the c-direction [63].

\subsubsection{Quantum EFA Calculations}

In tiberCAD, quantum mechanical models based on envelope function approximation (EFA) are used to calculate quantum properties such as eigenstates and eigenfunctions in quantum well structures. Module efaschroedinger solves schroedinger equation for electrons and holes. The generalized complex eigen value problem is given by [63],

$$
\mathrm{H} \psi=\mathrm{ES} \psi
$$

where, $\mathrm{H}$ and $\mathrm{S}$ are the Hamiltonian and S-matrix respectively.

The following options influence the behaviour of the Module efaschroedinger:

- Particle: specifies the particle for which the schroedinger equation is solved. Electrons (el) and holes (hl)

- Poisson model: provides electric potential

- Strain model: provides elastic strain

- Number of eigenstates: defines the number of eigenvalues and eigenfunctions to be found

- Dirichlet boundary conditions

- Solver for the eigenvalue problem

- Single band model and k . p model 


\subsection{Crosslight APSYS}

Crosslight software provides flexibility to design semiconductor devices and processes using several TCAD simulation packages such as LASTIP, PICS3D, APSYS, CSUPREM, PROCOM and NovaTCAD. LASTIP, PIC3D and APSYS are used to simulate optoelectronic devices whereas the other simulators serve as growth process modeling tools [65]. In this study, APSYS simulator was used to analyze the LED devices.

APSYS is a two-dimensional (2D) / three-dimensional (3D) simulator for modern semiconductor devices. It solves several advanced physical models based on finite element analysis. It can handle almost all semiconductor devices such as transistors, HEMTs, LEDs and Solar Cells except for lasers [65]. The physical models used in this study were:

- Drift-Diffusion Model

- Ray Tracing Model

- Quantum Well Models

This section mainly concentrates on quantum well transport, carrier scattering and recombination models. Drift-diffusion and ray tracing models can be referred in Sections 4.1 and 4.2.

\subsubsection{Quantum Well Models}

Quantum well models are among the most sophisticated models used in Crosslight Software and there are different levels of approximation available. They are summarized in Figure 4.4 and detailed below:

- The simple (also the default) quantum model assumes a single, symmetric, flat-band and stepwise potential profile. Quantum wells in a MQW region are assumed to be isolated from each other and the wave functions do not overlap.

- The complex MQW removes the restriction of symmetry and allows us to treat asymmetric wells (2a) or coupling between wells (2b).

- The self-consistent MQW model removes the restriction of at-band and couples the potential with charge density in a self-consistent manner. This can be used in isolated quantum wells (3a) or in a coupled complex MQW (3b). 


\section{Default: symmetric and isolated QW}

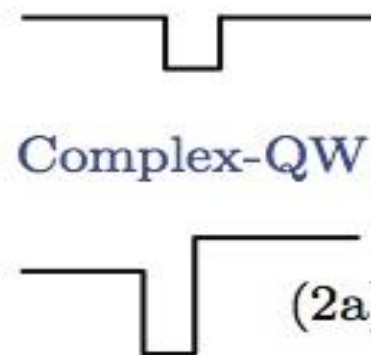

Self-consistent QW:

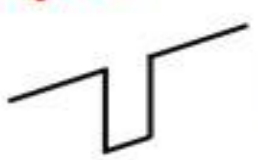

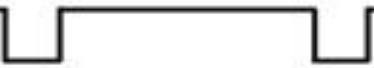

Complex-MQW:

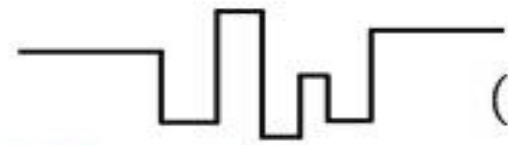

Self-consistent MQW:

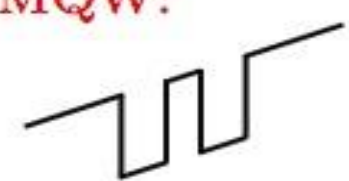

(2b)

(1)

$(3 \mathrm{~b})$

Figure 4.4: Quantum well models in Crosslight (Crosslight [65])

\subsubsection{Quantum Well Transport Models}

\subsubsection{Carrier Capture}

A ballistic transport approach is used to handle the carrier capture or escape into or out of a quantum well. It follows the treatment of Grupen and Hess [66]. This approach is illustrated in Figure 4.5.

The transport perpendicular to the QW plane is treated as follows. The QW is treated as a point source of recombination, which contains separate continuum and bound states. In this case, the continuum and bound states are assumed to have different quasi-Fermi levels that lead to separate continuity equations for the continuum and bound states. Transport of carriers from the regions outside the $\mathrm{QW}$ to the $\mathrm{QW}$ continuum states is by thermionic emission. The transition from the $\mathrm{QW}$ continuum to the bound states is computed by a scattering rate that includes carrier-carrier scattering. The bound states are solved from the Schrödinger equation. Within the quantum well in the direction parallel to the QW plane, drift-diffusion transport is assumed to be valid [54]. 


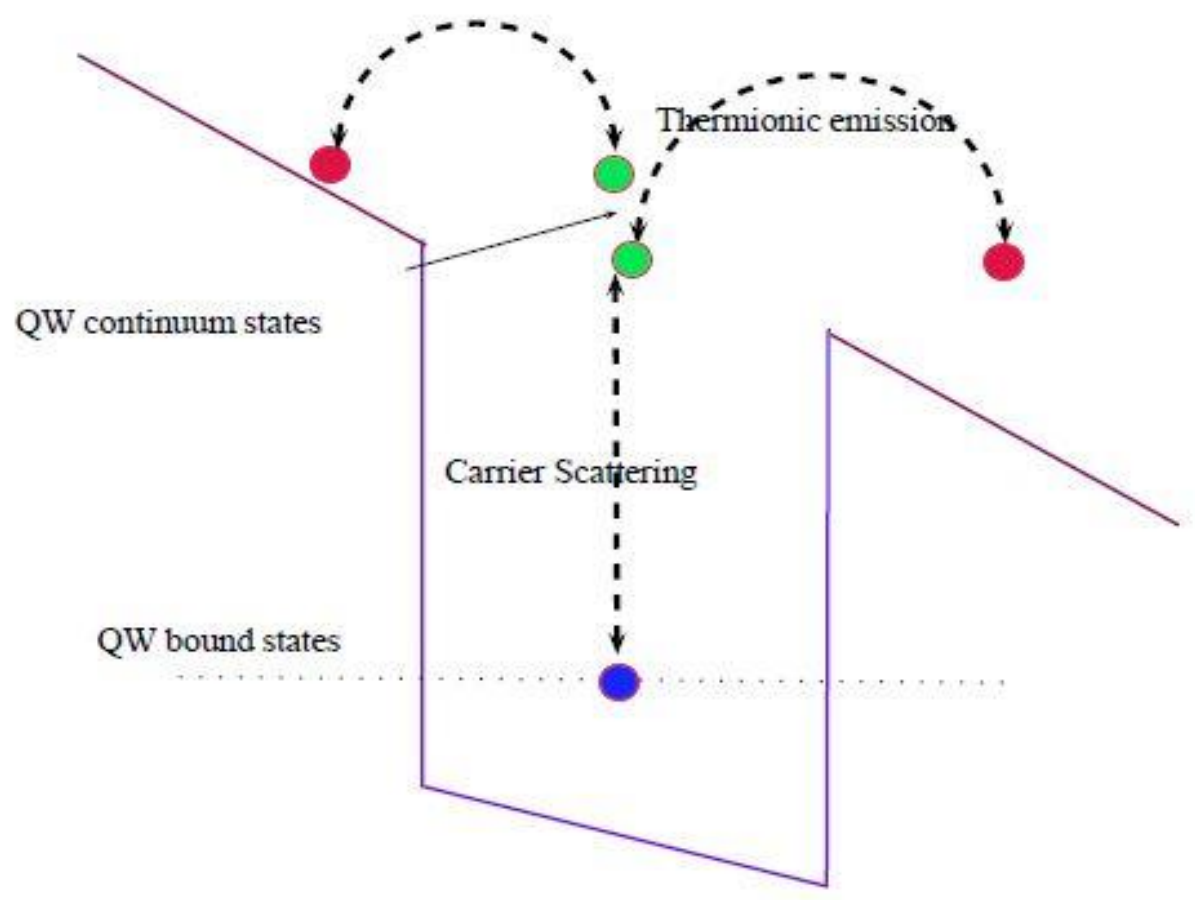

Figure 4.5: Discretization of quantum well to handle the physics of quantum well transport (Zbigniew Koziol, Sergey I. Matyukhin [67])

\subsubsection{Thermionic Emission}

Thermionic emission is used at the quantum well interface to handle the large momentum changes caused by the band-edge discontinuity. If thermionic emission is used only, without the QW scattering model, all the carriers are assumed to be totally captured in the quantum well. The default setting uses thermionic emission at the quantum well interfaces. This is activated by default if the keyword QWTransport is used in the Physics section of the command file.

The QW region must be identified by the keyword Active and must be discretized according to the constraints of the special 'three-point' QW model. The keyword QWTransport informs Sentaurus Device to use the 'three-point' QW model to treat the Active quantum well region [54].

\subsubsection{Recombination in Quantum Wells}

After the carriers are captured in the active region, they experience either dark recombination processes (such as Auger and $\mathrm{SRH}$ ) or radiative recombination processes (such as stimulated and spontaneous emissions), or escape from the active region. 


\subsubsection{Radiative Recombination}

Radiative recombination occurs in direct bandgap semiconductors since no momentum change is involved and is given as [63]:

$$
R_{\text {direct }}=C\left(n p-n_{i}^{2}\right)
$$

\subsubsection{Shockley-Read-Hall (SRH) Recombination}

'The nonradiative recombination through deep levels is called Shockley-Read-Hall recombination. The rate of single-level recombination is given by [29]:

$$
R_{S R H}=\frac{\sigma_{e} \sigma_{h} v_{t h} N_{T}\left(n p-n_{i}{ }^{2}\right)}{\sigma_{e}\left[n+n_{i} \exp \left(\frac{E_{T}-E_{F i}}{K_{B} T}\right)\right]+\sigma_{h}\left[p+n_{i} \exp \left(-\frac{E_{T}-E_{F i}}{K_{B} T}\right)\right]}
$$

where $\sigma_{e, h}$ is e and $\mathrm{h}$ capture cross-sections at the traps, $\mathrm{v}_{\text {th }}$ is the thermal velocity of carriers, $\mathrm{N}_{\mathrm{T}}$ is the trap density and $E_{T}$ is the energy position of the traps.

\subsubsection{Auger Recombination}

A three-carrier nonradiative recombination process in which the excess energy released by the recombination of e-h pair is transferred s kinetic energy to a third electron or hole, which is raised to a higher energy level within its respective band.

Auger recombination is important when excess carrier concentration is high. The rate increase with increasing doping concentration and temperature, and decreasing $E_{g}$. It limits the performance of narrow bandgap semiconductor lasers [29].

$$
R_{\text {auger }}=C n p^{2} \text { or } R_{\text {auger }}=C n^{2} p
$$

where $\mathrm{C}$ is the Auger coefficient of the semiconductor. 


\section{Chapter 5 Modeling Results}

This chapter presents a systematic study of the design and analysis of multiple quantum well LEDs to investigate the influence of quantum wells on the performance of the LEDs. Several TCAD tools and their effectiveness in analyzing devices have been evaluated.

\subsection{Modeling Strategy}

In this thesis, TCAD models were developed to analyze Light Emitting Diode characteristics at an early stage itself thereby saving cost and time involved in manufacturing. First step in any modeling strategy is to design the device and generate a suitable mesh grid. The device parameters such as layer thicknesses, molefractions and doping concentrations were provided by growth engineers and material parameters such as band gaps and electron affinities have been obtained from the literature. Next step involved incorporating relevant physical models into the device structure. The physical models used in this study were explained in Chapter 4. After analyzing both simulated and experimental Light Emitting Diode characteristics, feedback was provided to the growth and fabrication engineers on what changes could be made to improve the internal and the external efficiencies of our device. Figure 5.1 shows the TCAD model flow in detail.

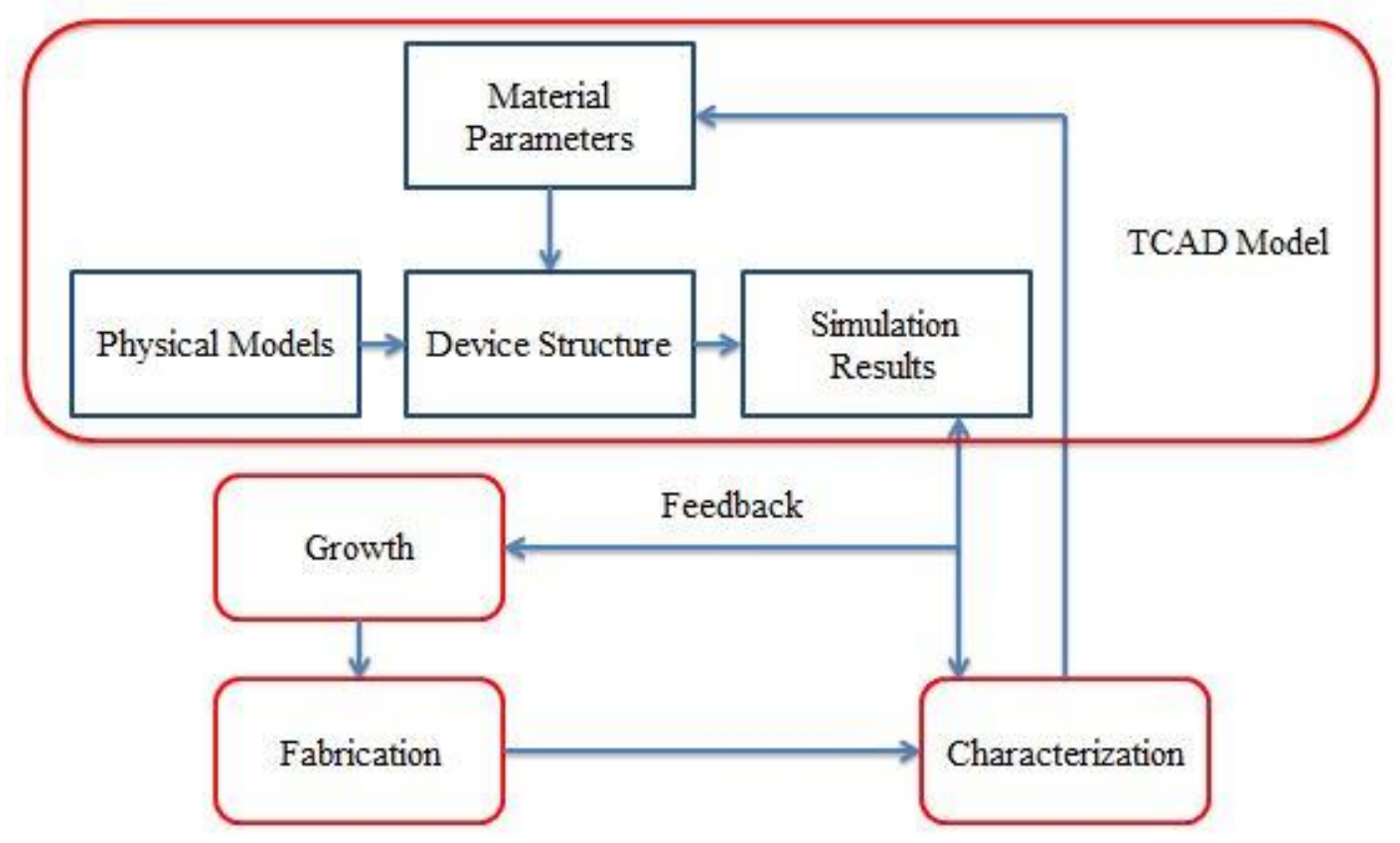

Figure 5.1: TCAD Model Design Flow 
In this thesis, Device CAD tool was implemented to study and improve LED devices. Figure 5.2 shows how a device CAD tool works.

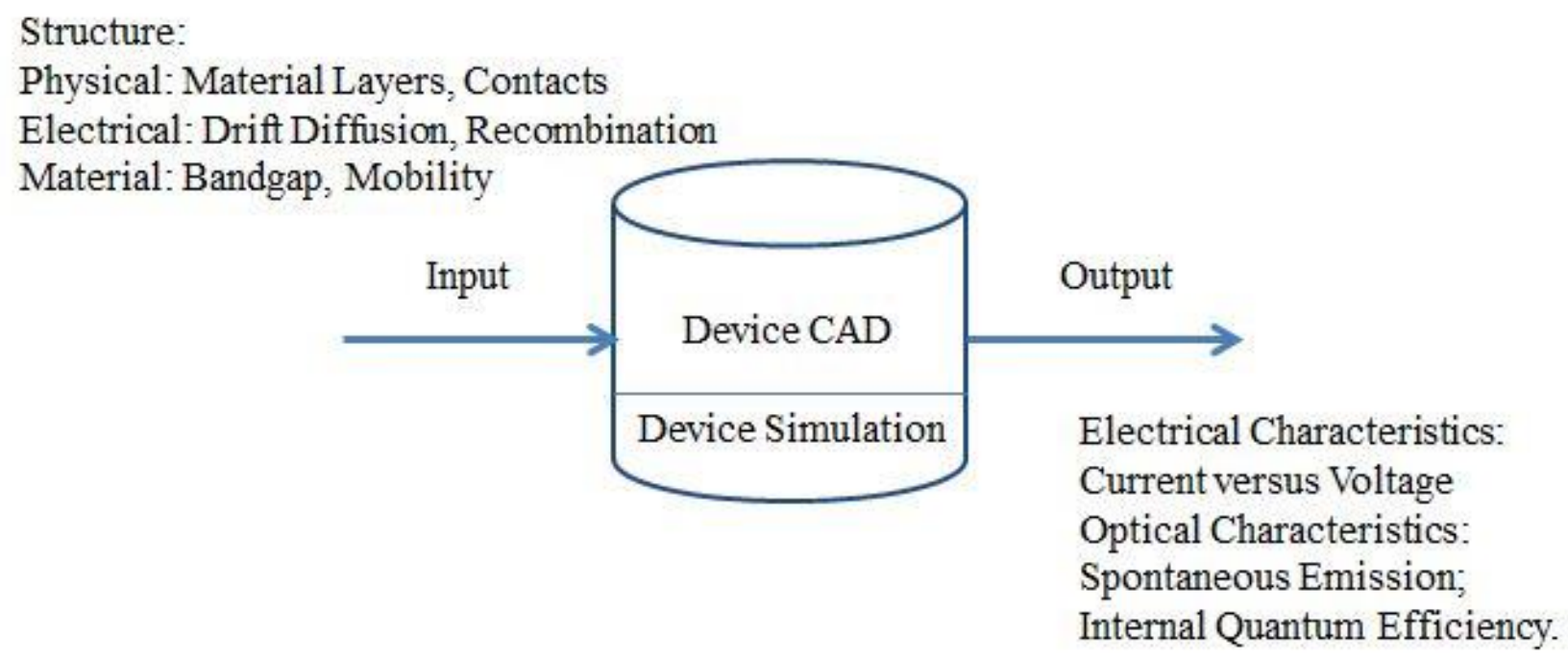

Figure 5.2: Device CAD

\subsection{Device Structure and Material Parameters}

The LED structure used in this study was a planar LED with ohmic contacts as shown in Figure 5.1. The structure employed for modeling was developed taking into account the specifications from WVU fabricated LED. $p$-AIGaN layer was used as an electron blocking layer (EBL) to improvise the number of possible radiative recombinations in quantum wells thereby increasing the emitted optical power. AIN acts as a nucleation layer (NL) required for optimal growth i.e. to avoid the lattice mismatch between gallium nitride and substrate.

The changes to previous structure and present structure was the $p$-GaN layer thickness. Present structure has a thickness of $140 \mathrm{~nm}$ which is double the value of previous structure $p$-GaN layer. This device was initially analyzed at different well to barrier (W/B) ratios, but considering the accurate results obtained at 0.355 (W/B), this value was continued in later parts of the study. 


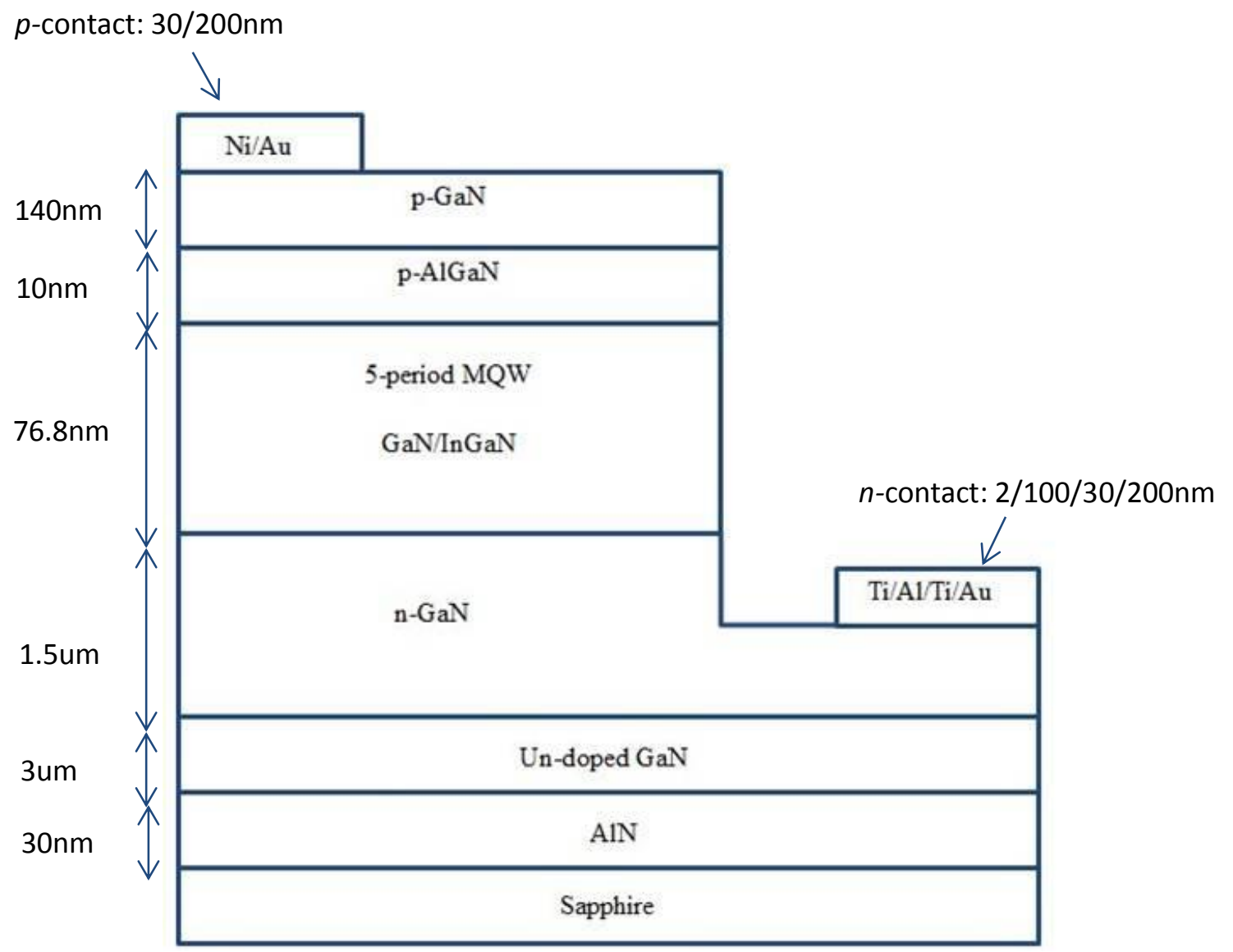

Figure 5.3: LED structure employed for modeling

As mentioned earlier, the material parameters have been either experimentally extracted from our MOVPE grown samples or obtained from the literature. The carrier concentrations of different materials have been determined from the Hall effect and CV measurements and the Quantum Well periodicity was extracted from XRD measurements. Since, the main focus of this study was optical optimization of LED devices, it was required to understand each layer behavior optically. Using Ellipsometry measurements, refractive indices were obtained and used in the TCAD models. All the material parameters of each layer were mentioned in the Appendix section for a reference. Figure 3.4 shows few of the parameters used in this study. 


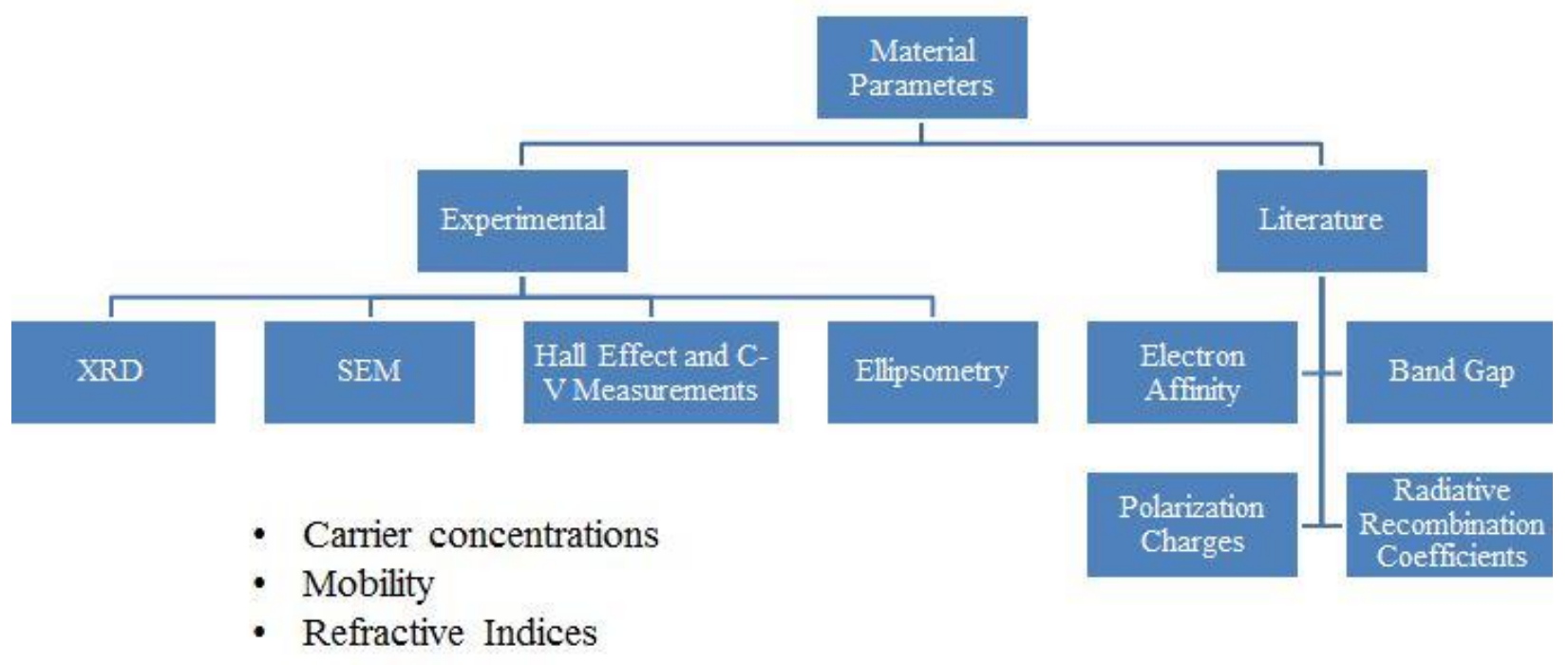

Figure 5.4: Material parameters used in this study

\subsection{LED study using Synopsys Sentaurus}

In the beginning of this thesis, Synopsys Sentaurus Workbench (SWB) was used to develop TCAD models to study the optical properties of LED devices. Figure 5.5 shows the work environment of Synopsys TCAD tool.

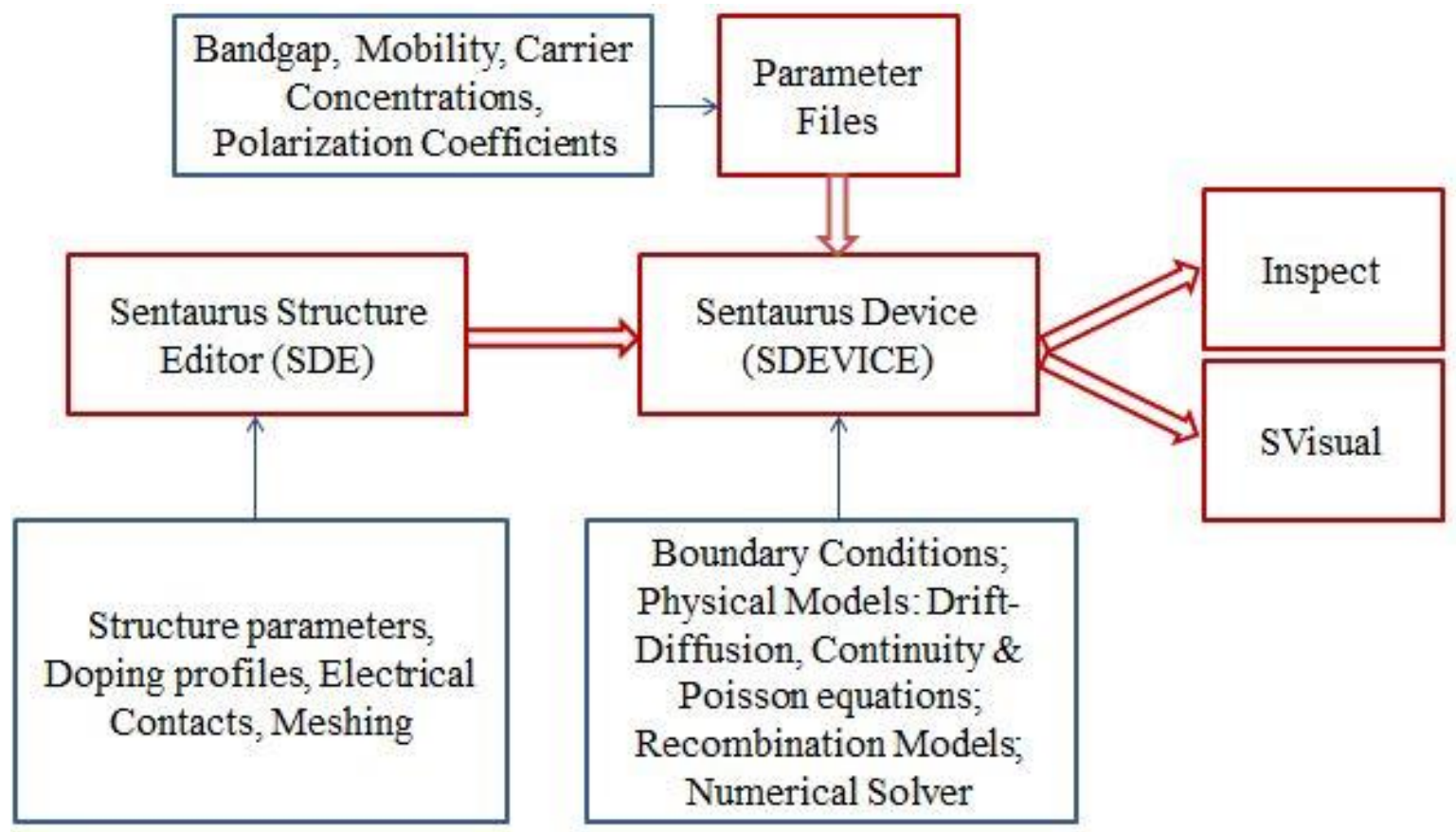

Figure 5.5: Synopsys Sentaurus Workbench 
Before implementing the physical models for optical study, the model was validated for electrical properties of LED devices. The band diagram of the LED structure is shown in Figure 5.6. It shows the electron blocking layer (EBL) and five quantum wells in the band structure. It is evident from the band structure that, a $p$-AIGaN EBL layer would have a bump in the conduction band acting as the barrier for the electron flowing from the active layer to the $p-\mathrm{GaN}$. Thus there could relatively be more electrons and holes confined in such a structure.

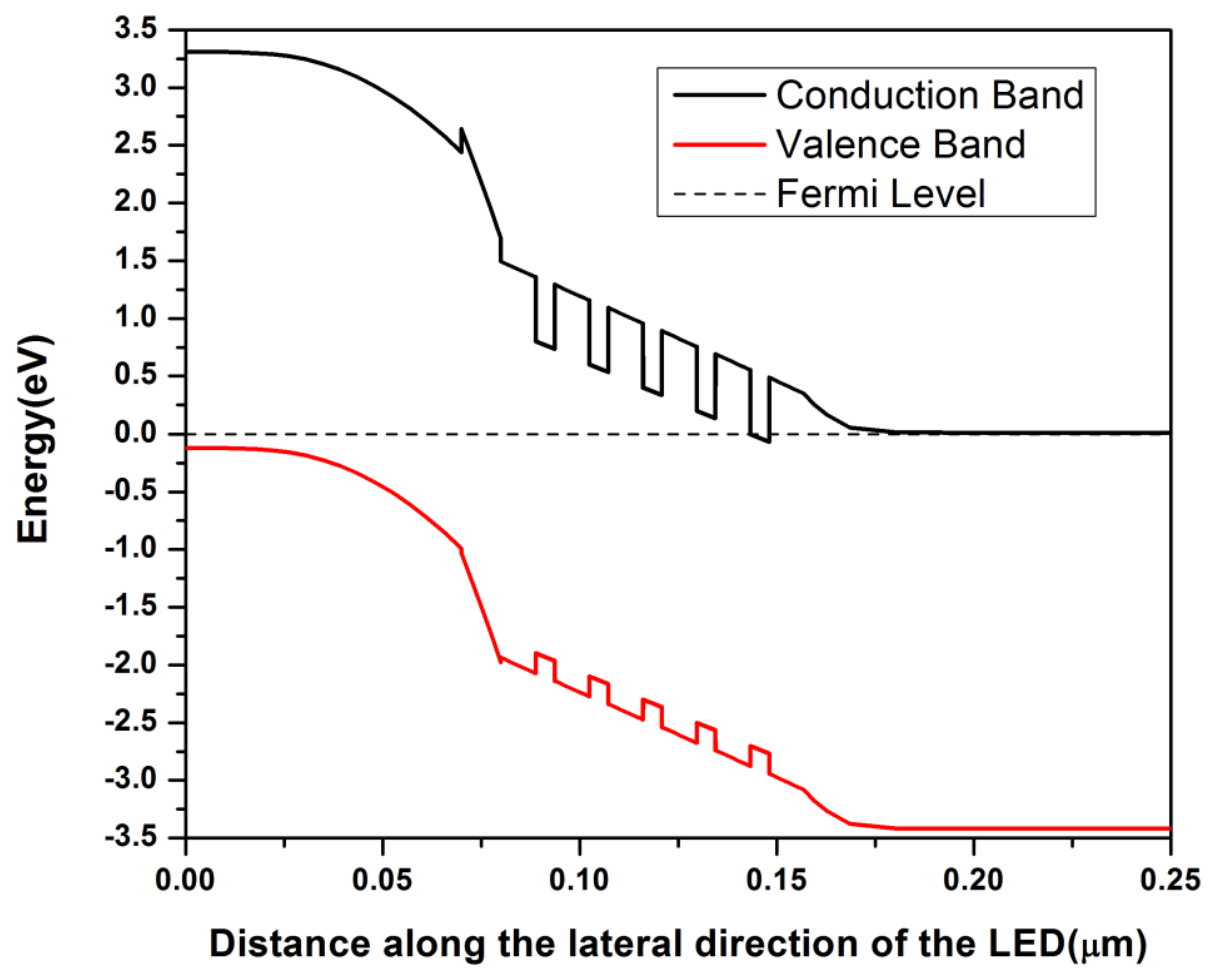

Figure 5.6: Structure and Band Diagram of the LED device plotted along the vertical direction

Then, TCAD model was developed for a single quantum well to study the PL Spectrum and the energies and wavelengths corresponding to each quantum well. A single quantum well structure is shown in Figure 5.7. But the PL spectra results obtained were not accurate when compared to the experimental data. From the analysis, we could found out that Synopsys doesn't support quantum well transport models for active devices. Figure 5.8 shows both the simulated and experimental results of PL spectra for GaN/InGaN quantum well. 


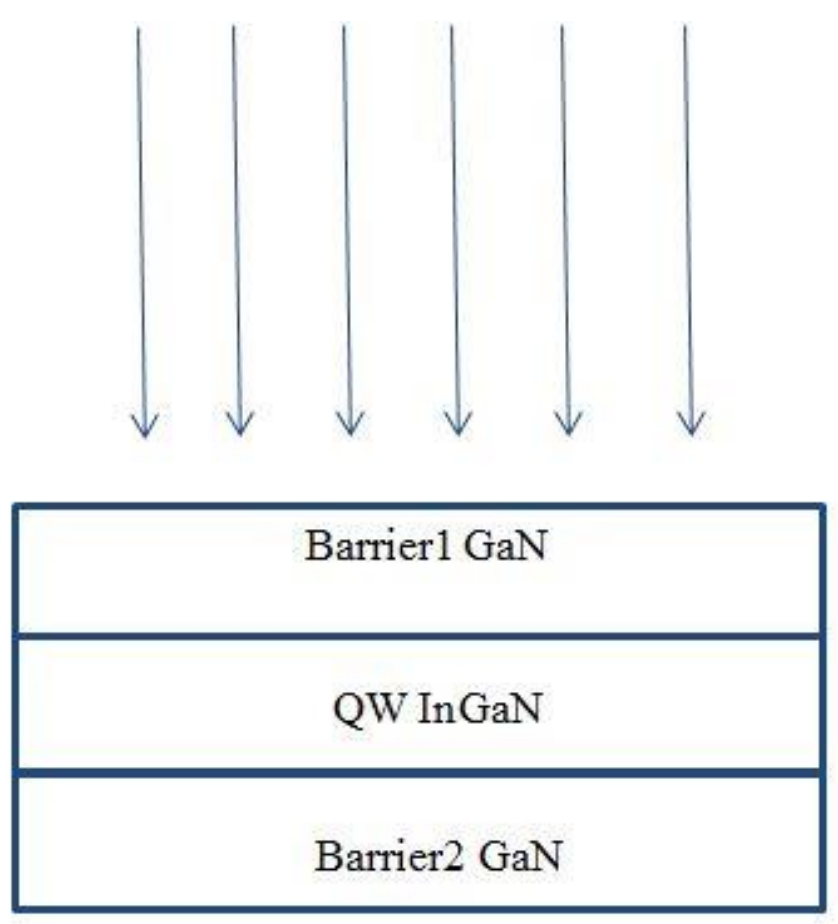

Figure 5.7: Structure used to study optical characteristics
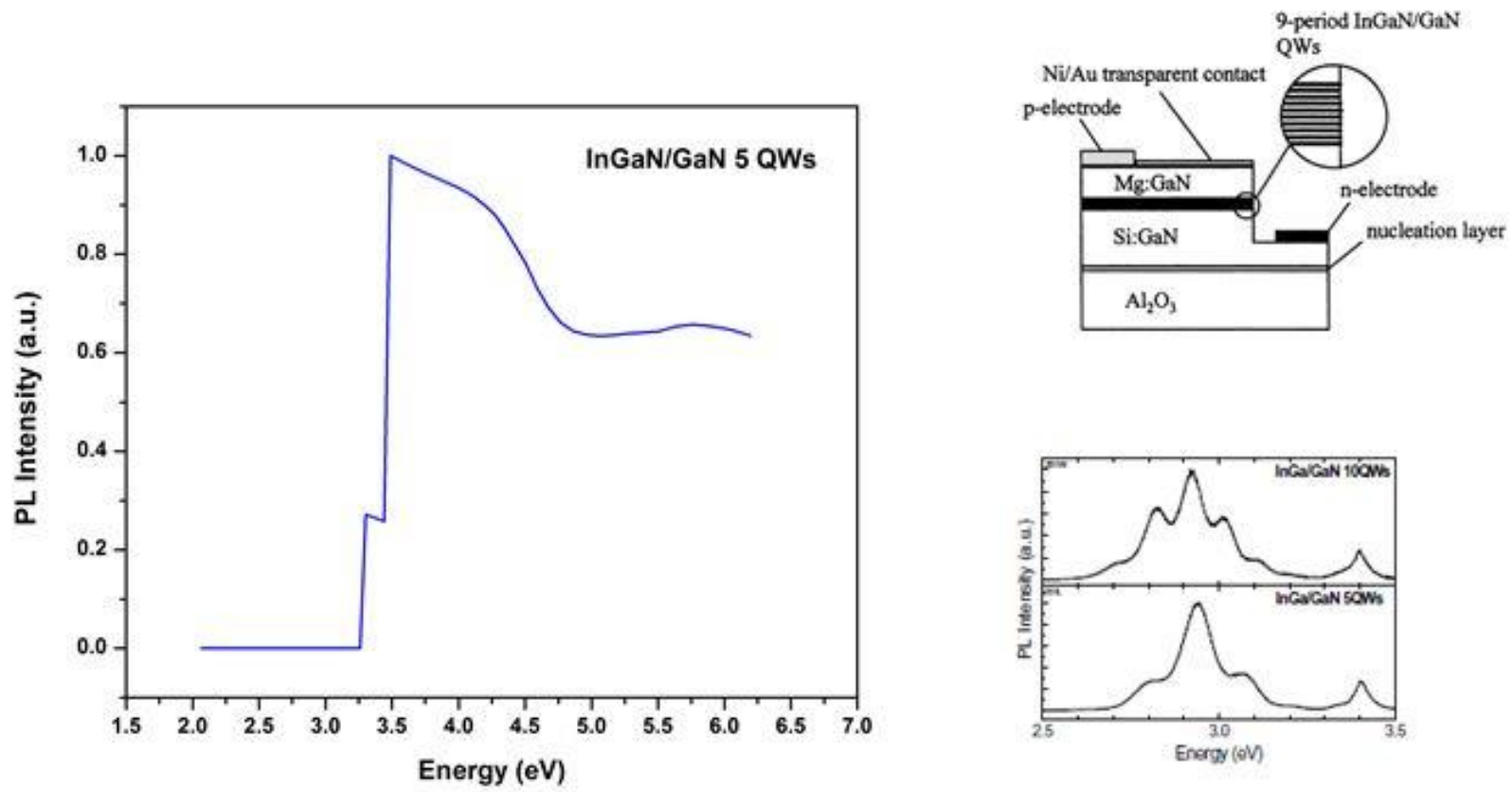

Figure 5.8: (a) Photoluminescence Spectrum (b) Experimental PL Spectrum from Literature (Zhe Chaun, Jeng-Hung Chen [68]) 


\subsection{Study of Quantum Properties of a single GaN/InGaN QW (1D) using tiberCAD}

In order to understand the influence of quantum well on LED device characteristics, first it is necessary to have a deep level understanding of each quantum well behavior in the active region. In this thesis, tiberCAD simulation tool was used to model and design quantum well structures. tiberCAD allows to calculate quantum properties of a single QW or multiple QW by taking into account the most important physical concepts such as quantum confinement, self-heating and polarization in heterostructures. Figure 5.10 shows a flow chart of how tiberCAD tool works.

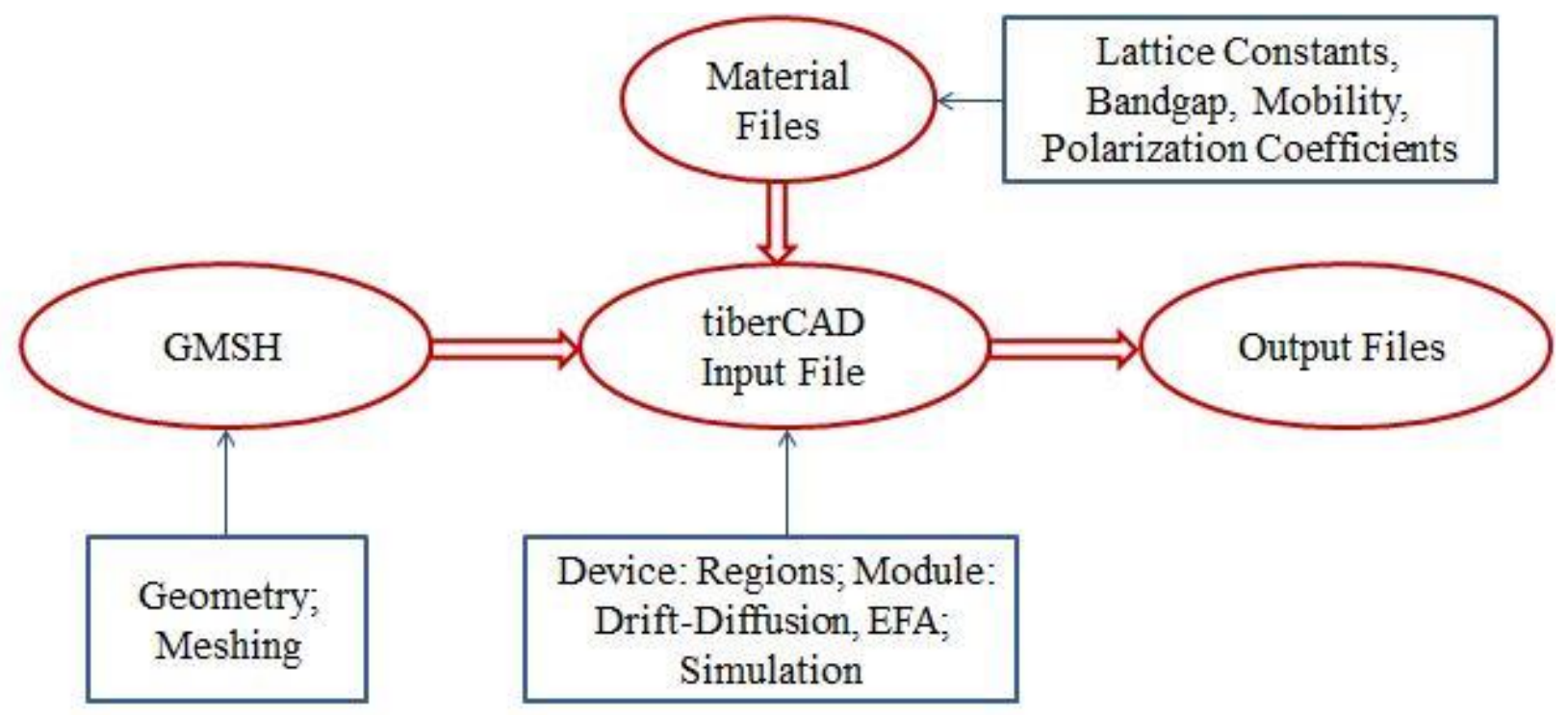

Figure 5.9: tiberCAD Flow Chart

The structure used for the study of quantum properties is shown in Figure 5.11 (a). Schrödinger equation was solved, with a single-band effective mass model for conduction band and with a 6 band-kp model for valence band to obtain the quantum properties. Eigenvalues and eigenfunctions are calculated to get energy levels and wavefunctions in the quantum well. The band structure shown in Figure 5.11 (b) was obtained at equilibrium. The band bending that can be observed is due to the default polarization effects present in the nitride materials. 


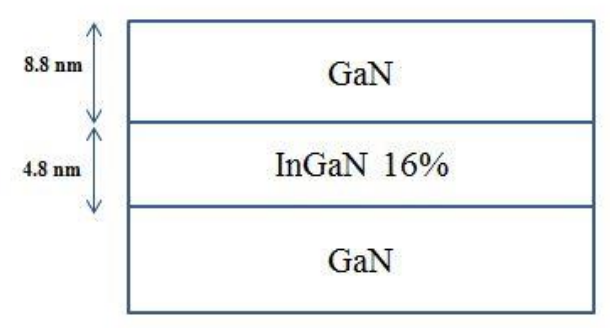

QW Periodicity Extracted from $X R D$

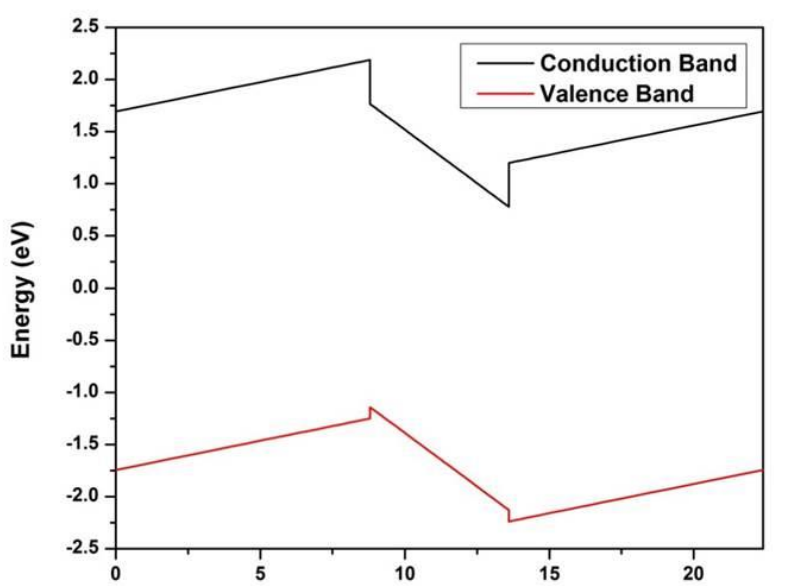

Distance along the lateral direction of the Quantum Well Structure (nm)

Figure 5.10: (a) Single GaN/InGaN QW structure used for modeling (b) Band structure at equilibrium

WVU fabricated LEDs are tested at a 5V forward bias and these LEDs operate as Photodiodes at a reverse bias voltage of $5 \mathrm{~V}$. So, it is required to study the behavior of a single quantum well band structure at different biases. Figure 5.12 shows influence of bias on quantum well band structure.

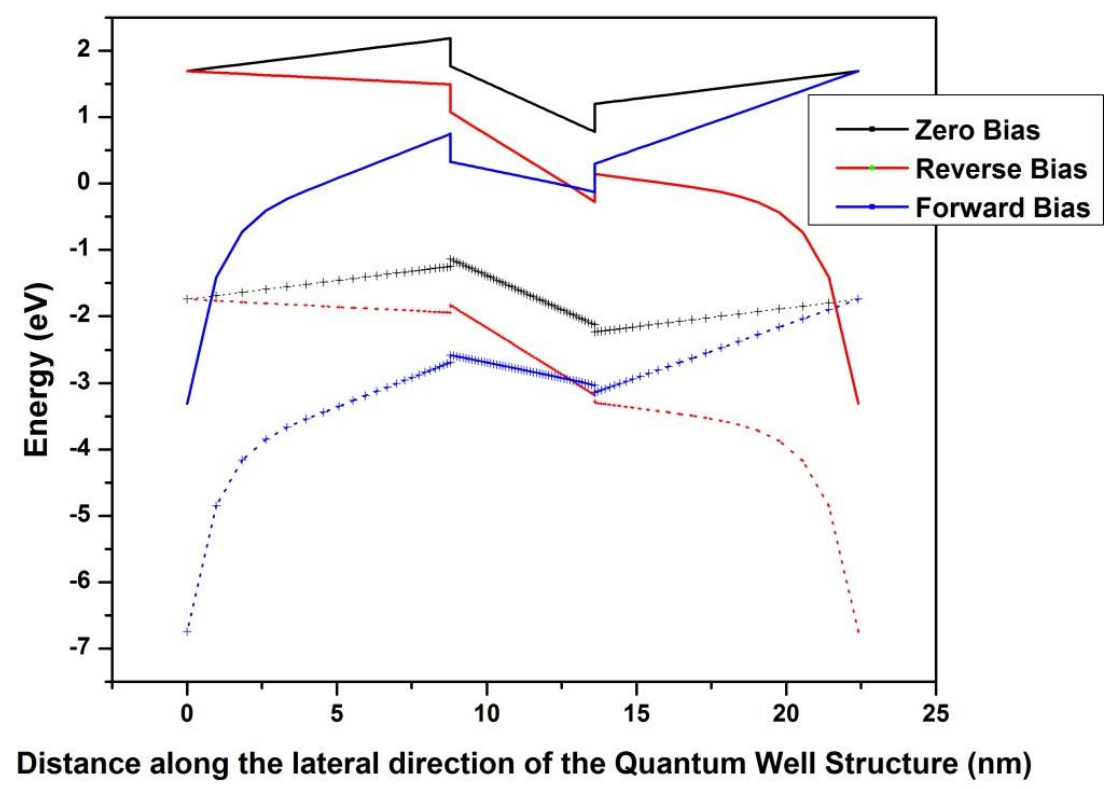

Figure 5.11: Band structure of a SQW at different biases 
tiberCAD tool provides energy levels and wavefunctions confined within a quantum well structure. Figure 5.13 shows energy levels confinement in conduction band and valence band at equilibrium. It can be observed six energy levels in conduction band and four in valence band were confined in a single quantum well with a periodicity of $12 \mathrm{~nm}$. Energy levels can be obtained in a similar way at desired forward and reverse bias voltages.

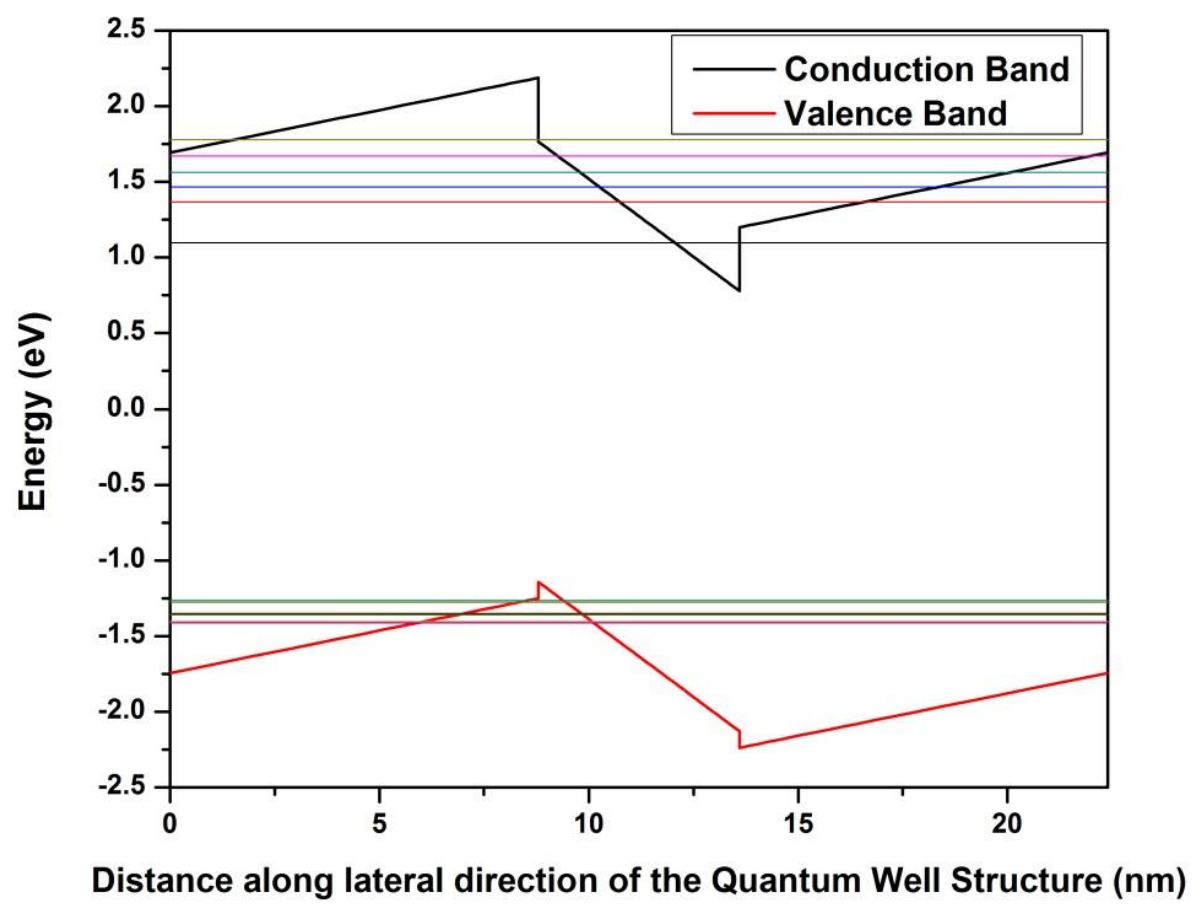

Figure 5.12: Energy levels confined in conduction and valence bands at equilibrium.

In order to verify the accuracy of the tool, the tool was validated by comparing the simulated and experimental data of an AlGaAs quantum well. Since data for an InGaN quantum well was not available due to the limited resources, the experimental PL spectra data for Arsenide materials was taken from the literature [67]. From Figure 5.14, the experimental and simulated energy levels at different quantum well widths of an AIGaAs quantum well were almost same. 

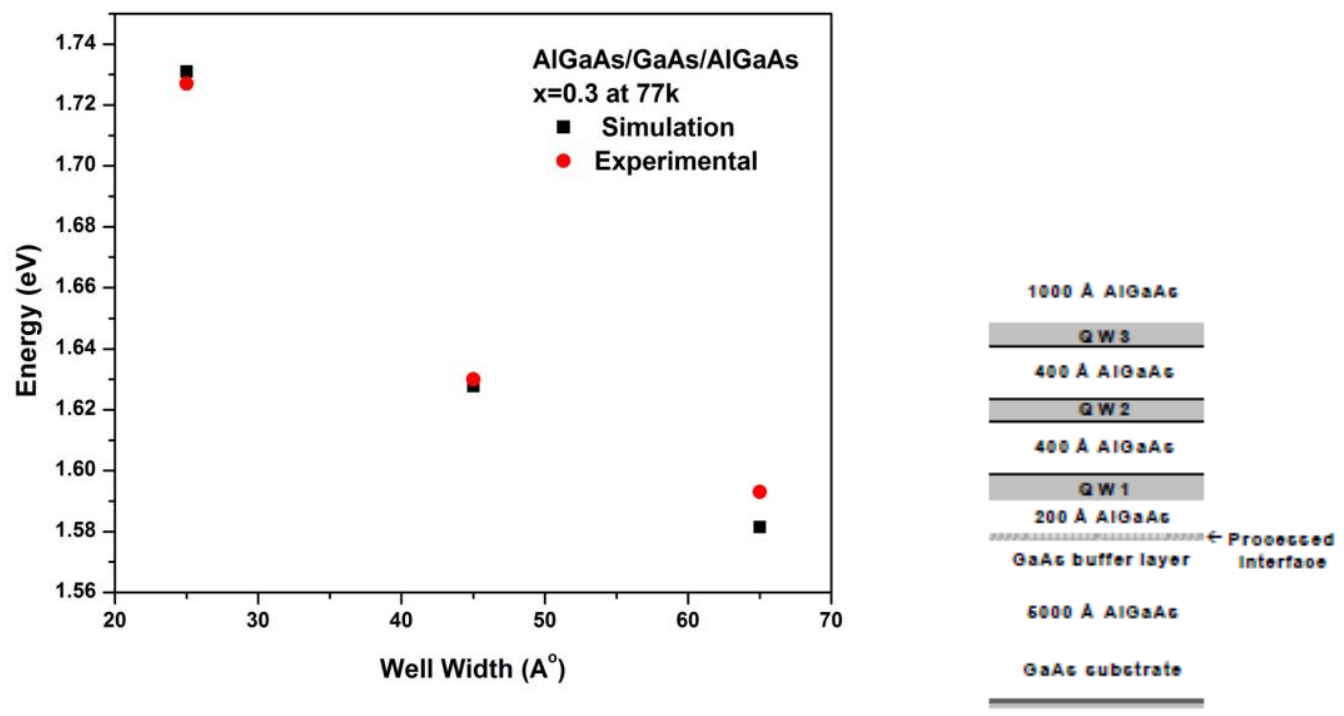

Figure 5.13: Verification and Validation (Yuan Zhi-liang, Xu Zhong-ying [69])

The influence of different quantum well widths and molefractions on energy levels and wavelengths was observed. It can be observed in Figures 5.15 and 5.16. Table 5.1 and 5.2 show the comparison of simulated and experimental energy levels and wavelengths at desired thickness $4.8 \mathrm{~nm}$ and molefraction $16 \%$.

Table 5.1: Influence of Quantum Well Width

\begin{tabular}{|c|c|c|}
\hline Comparison & Energy (eV) & Wavelength (nm) \\
\hline Simulation & 2.72 & 455.5 \\
\hline Experimental & 2.7482 & 445 \\
\hline
\end{tabular}

Table 5.2: Influence of Quantum Well Molefraction

\begin{tabular}{|c|c|c|}
\hline Comparison & Energy (eV) & Wavelength (nm) \\
\hline Simulation & 2.705 & 458 \\
\hline Experimental & 2.7482 & 445 \\
\hline
\end{tabular}




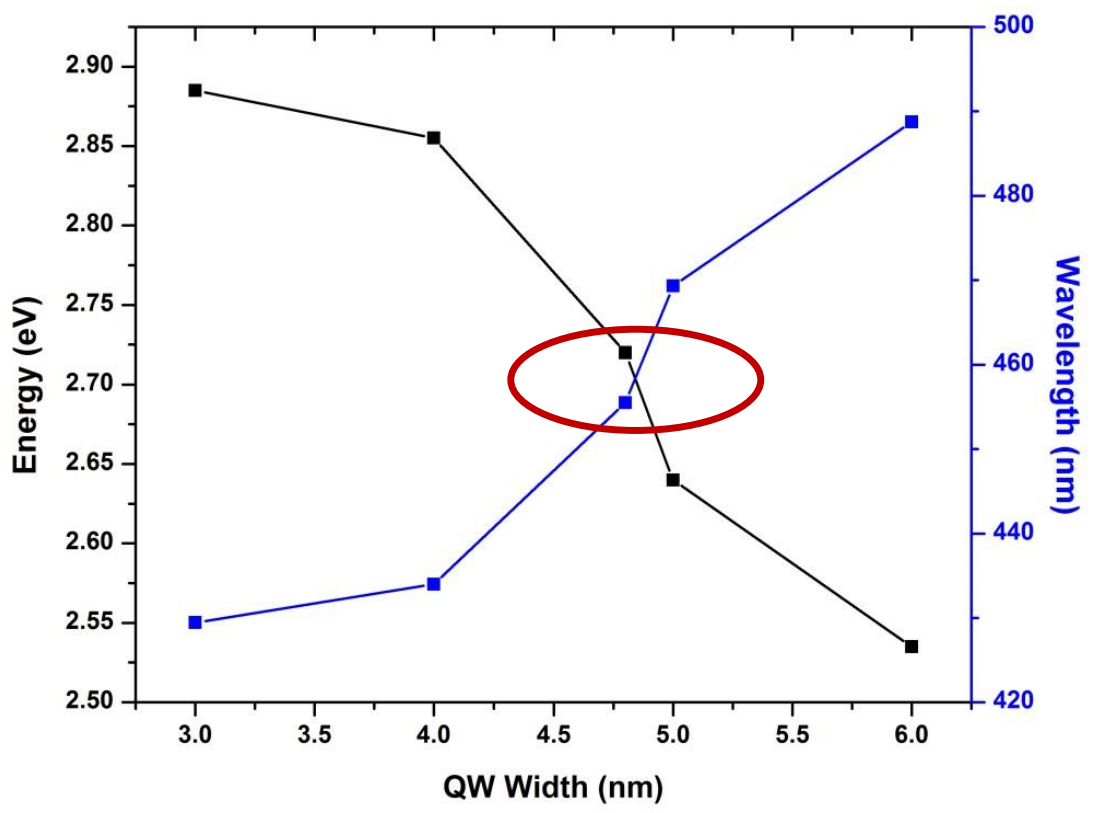

Figure 5.14: Influence of quantum well widths on energy levels of a single quantum well at forward bias

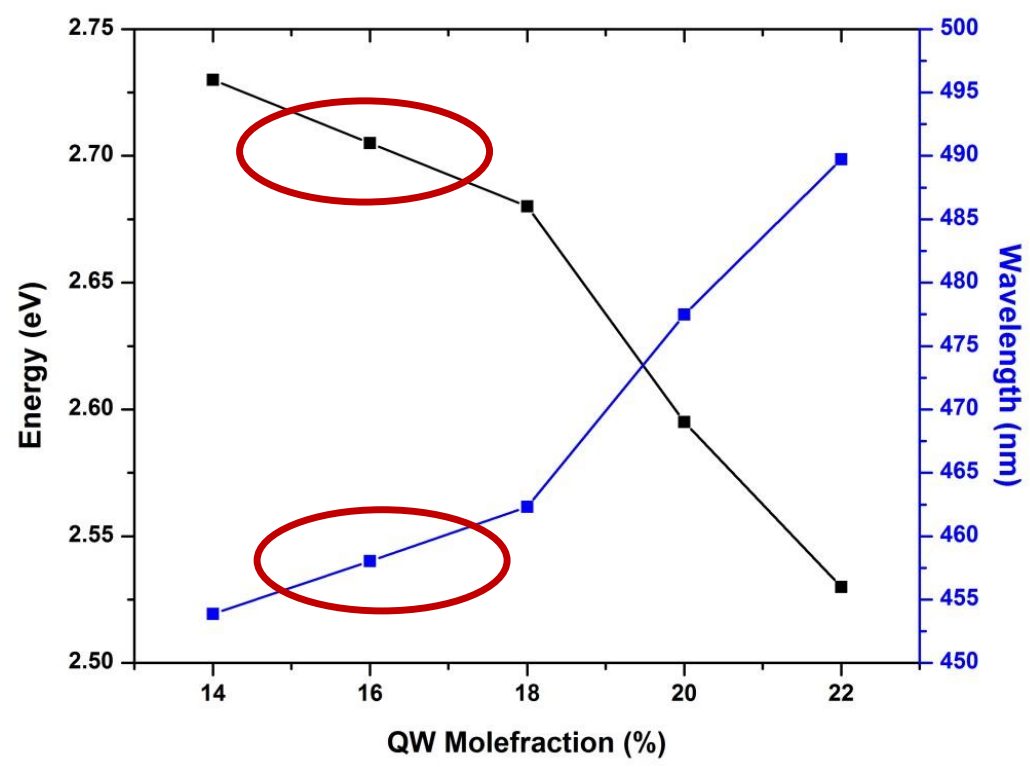

Figure 5.15: Influence of quantum well molefraction on energy levels of a single quantum well at forward bias 


\subsection{Study of Influence of Quantum Well on LED Characteristics using Crosslight APSYS}

To study quantum well influence on LED device characteristics, it is important to use TCAD tools that support quantum well models. Crosslight APSYS tool provides the required carrier scattering and transport models. Figure 5.17 shows the flow chart of a Crosslight TCAD tool.

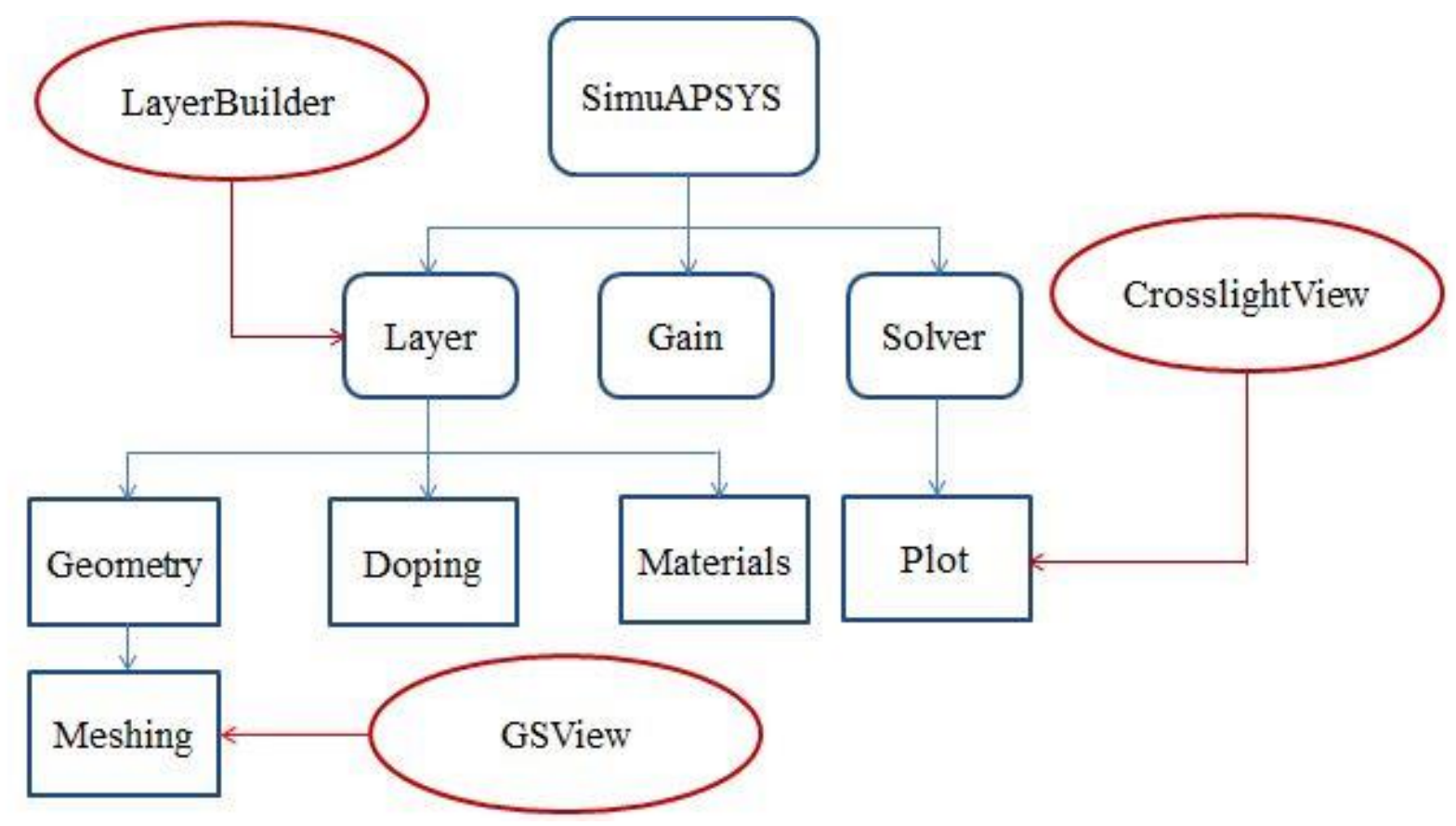

Figure 5.16: Crosslight APSYS Flow Chart

In the beginning, electrical properties such as band diagram was studied and analyzed. This tool provides the wave functions within a quantum well along with the band structure. Figure 5.18 (a) shows the band structure of a 5 quantum well LED without polarization effects included and (b) with polarization included. Polarization field tilts the energy band and induces the spatial separation of the electron and hole wave functions. 


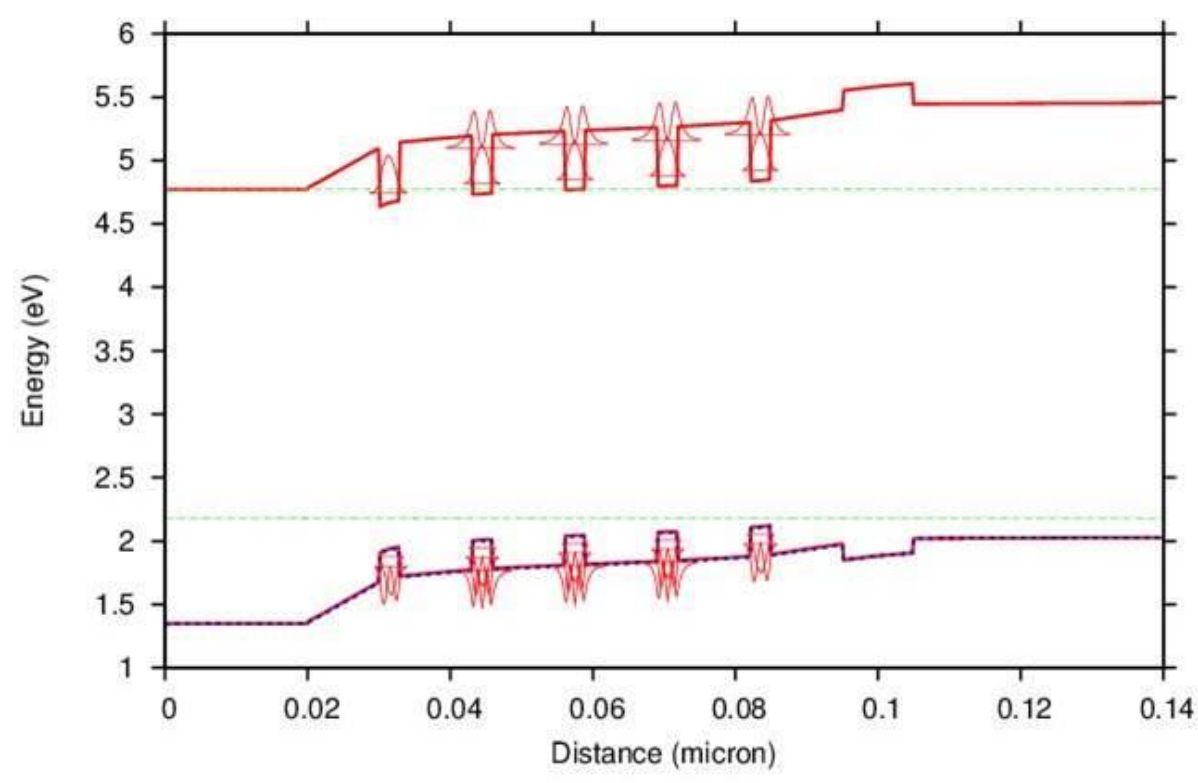

(a)

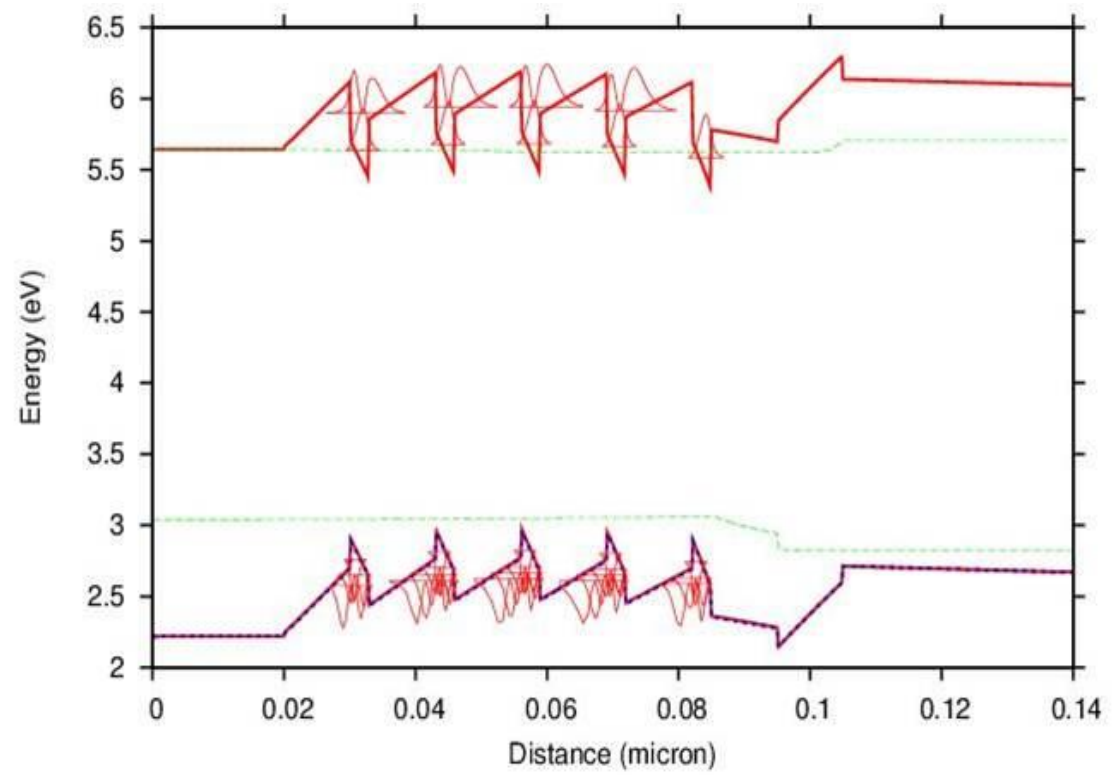

(b)

Figure 5.17: Band diagram and Wavefunctions of InGaN LED (a) Non-polarized (b) Polarized

The spontaneous emission obtained from the simulations was compared with the experimental results and analyzed. The peak wavelengths were almost same with a difference of $3 \mathrm{~nm}$. The tail on the right side of the experimental EL Spectrum is due to the density of states. These results were for a sample of $12 \mathrm{~nm}$ quantum well periodicity grown in WVU. 


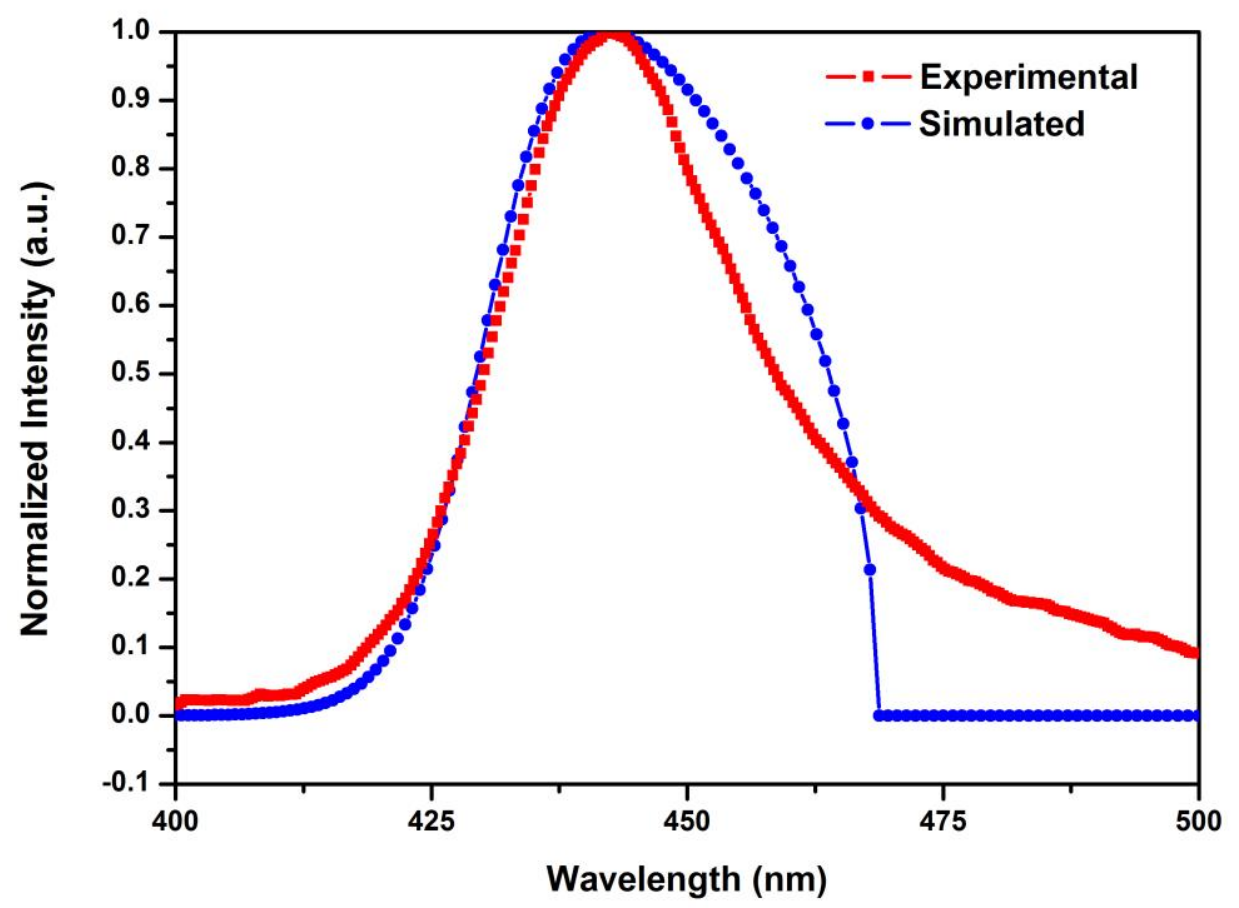

Figure 5.18: Comparison of Experimental and Simulation Electroluminescence Spectra results

Table 5.3: EL Spectra experimental and simulation comaprison

\begin{tabular}{|c|c|c|}
\hline Comparison & Energy $(\mathrm{eV})$ & Wavelength $(\mathrm{nm})$ \\
\hline Simulation & 2.8031 & 442 \\
\hline Experimental & 2.7482 & 445 \\
\hline
\end{tabular}

El spectrum at various molefractions was obtained. The WVU LED devices have $16 \% \operatorname{InGaN}$ as quantum wells. Through simulations, effect of change in molefraction on EL Spectra was studied. Figure 5.20 shows normalized intensity plots for $16 \%$ and $18 \% \operatorname{InGaN}$ concentration and a red shift was observed in the peak wavelength. 


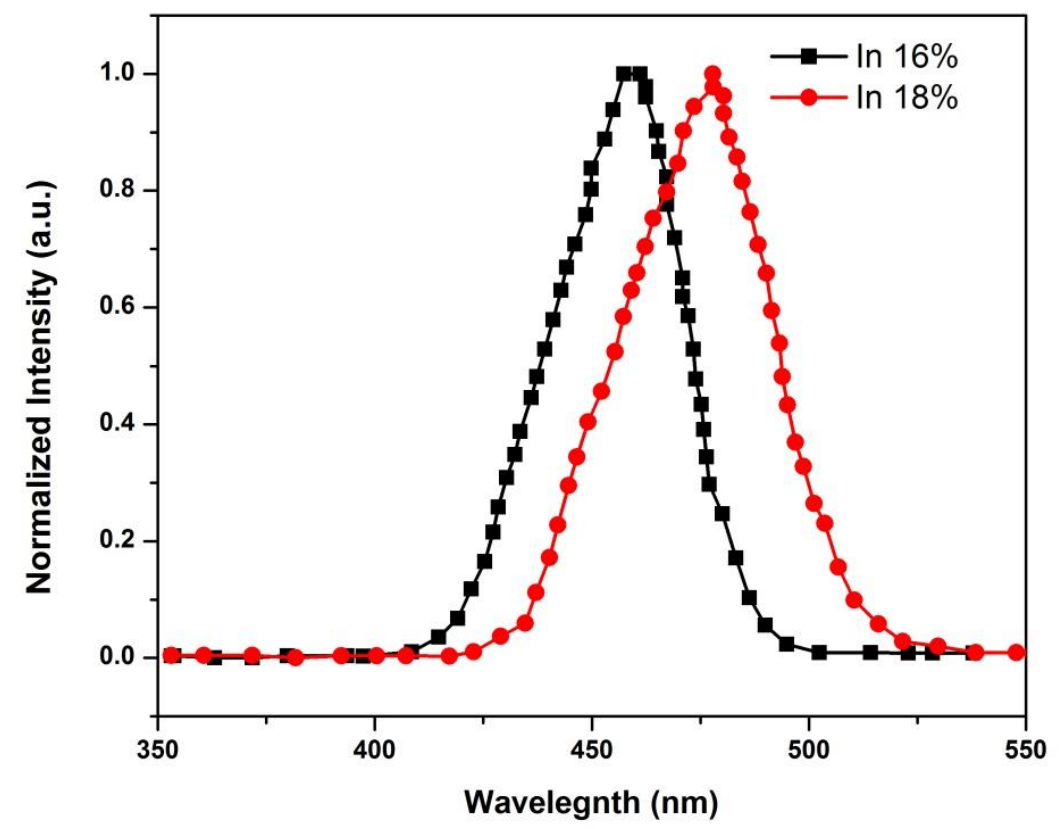

Figure 5.19: Influence of quantum well molefraction on EL Spectra 


\section{Chapter 6 Conclusions and Future Work}

This thesis presented important findings related to the development of GaN-based lightemitting diodes. Below is the summary of significant results and conclusions. In addition, the readers of this thesis may be interested in knowing some suggestions for future work. Therefore, I summarize promising directions for future work based on my work.

\subsection{Conclusions}

In this study, several TCAD tools have been used to study quantum well properties and electrical and optical characteristics of light emitting diodes. First, Synopsys Sentaurus TCAD tool has been used to study the effect of quantum wells on LED performance. Electrical characteristics such as band structure were obtained and analyzed but optical characteristics such as PL Spectra results were not accurate After analyzing the TCAD model and contacting Synopsys support group, it was found out that this TCAD tool does not support required quantum well transport models for active devices.

Before moving to another device level TCAD tools, understanding quantum well behavior at an active region level was important. tiberCAD tool was used to study the quantum properties of a $\mathrm{GaN} / \mathrm{InGaN}$ single and multiple quantum well structure and the influence of quantum well properties on LED performance. Schrodinger equation was solved to obtained energy levels and wavefunctions of the quantum wells. These results were analyzed at different quantum well widths and molefractions. The tool was also validated by using experimental data from the literature to check for accuarcy. It was observed that the simulation results were a close match to the experimental results.

Crosslight APSYS tool supported the quantum well transport and carrier scattering models and hence was used to study LED characteristics. It also gave wavefunctions corresponding to each quantum well along with the band structure. EL Spectra of a $12 \mathrm{~nm}$ QW periodicity LED sample was obtained through simulations and was compared with the experimental normalized intensity. The peak wavelengths were almost same with a $3 \mathrm{~nm}$ shift. Influence of QW molefractions on EL Spectrum were studied and a red shift in peak wavelength was observed. Table 6.1 gives an overview of each tool and its capability to study device characteristics. 
Table 6.1: Comparision of Simulation Tools

\begin{tabular}{|c|c|c|c|}
\hline TCAD Tools & Synopsys Sentaurus & tiberCAD 2.0 & Crosslight APSYS \\
\hline Meshing & Manually & GMSH & SimuAPSY \\
\hline $\begin{array}{c}\text { Physical Models for } \\
\text { Electrical Optimization }\end{array}$ & Yes & Yes \\
\hline $\begin{array}{c}\text { Quantum Well } \\
\text { Transport Models }\end{array}$ & No & Yes & Yes \\
\hline Carrier Scattering \\
Models
\end{tabular}

\subsection{Future Work}

Advancement to the model can be made by including the trap charges in simulations. The device structure considered in this study was an ideal device without including the trap densities and locations. Trap densities and cross section values can be obtained from Deep Level transient spectroscopy (DLTS) set up established at WVU. The feedback from the TCAD models after including traps can be provided to the growth and fabrication engineers to obtain optimal growth.

Crosslight APSYS tool can be used to obtain PL spectra results and compare with the experimental results of PL. A PL set up can be established in future to obtain the experimental results. 


\section{Appendix A}

\section{Design of Multiple Quantum Well LED devices using Sentaurus Workbench}

\section{A.1 Sentaurus Structure Editor}

; sde_dvs.cmd

(sde:clear)

\#set wfront 0

\#set wtot 200

(define node “@node@”)

\#set xmole_InGaN_well 0.16

(define xm1@xmole_InGaN_well@)

(define t_QW 0.0088)

(define t_B 0.0048)

(define t_topstructure (+ t_B t_QW t_B))

; general structure parameters

(define width 200); total width of the top part (mesa) of structure

(define regions (list "Barrier0" "QW1" "Barrier1"))

(define materials (list "GaN" "InGaN" "GaN"))

(define dopings (list 000 ))

(define heights (list t_B t_QW t_B))

(define widths (list width width width))

(define xmoles (list 0 xm1 0))

(display "

$-\left(n^{\prime \prime}\right)$

(display "Defining structure generation procedures that are called later $\backslash n$ ")

(display “ $-\left(n^{\prime \prime}\right)$

; create a constant region-based doping profile where negative doping = p-type!!

(define layer:create-doping

(lambda (name doping)

(if (< doping 0) (define dopingtype "Magnesium") 


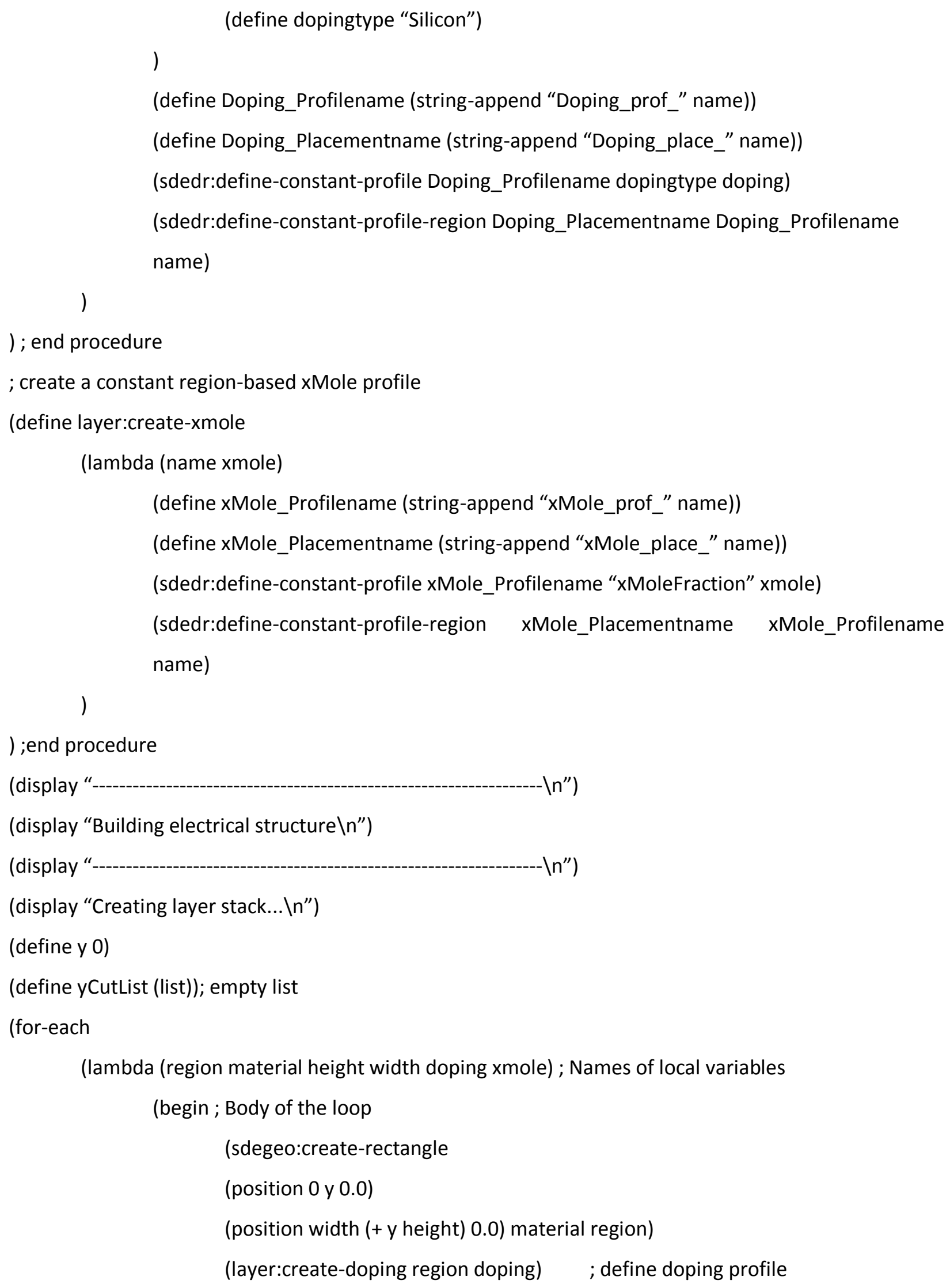




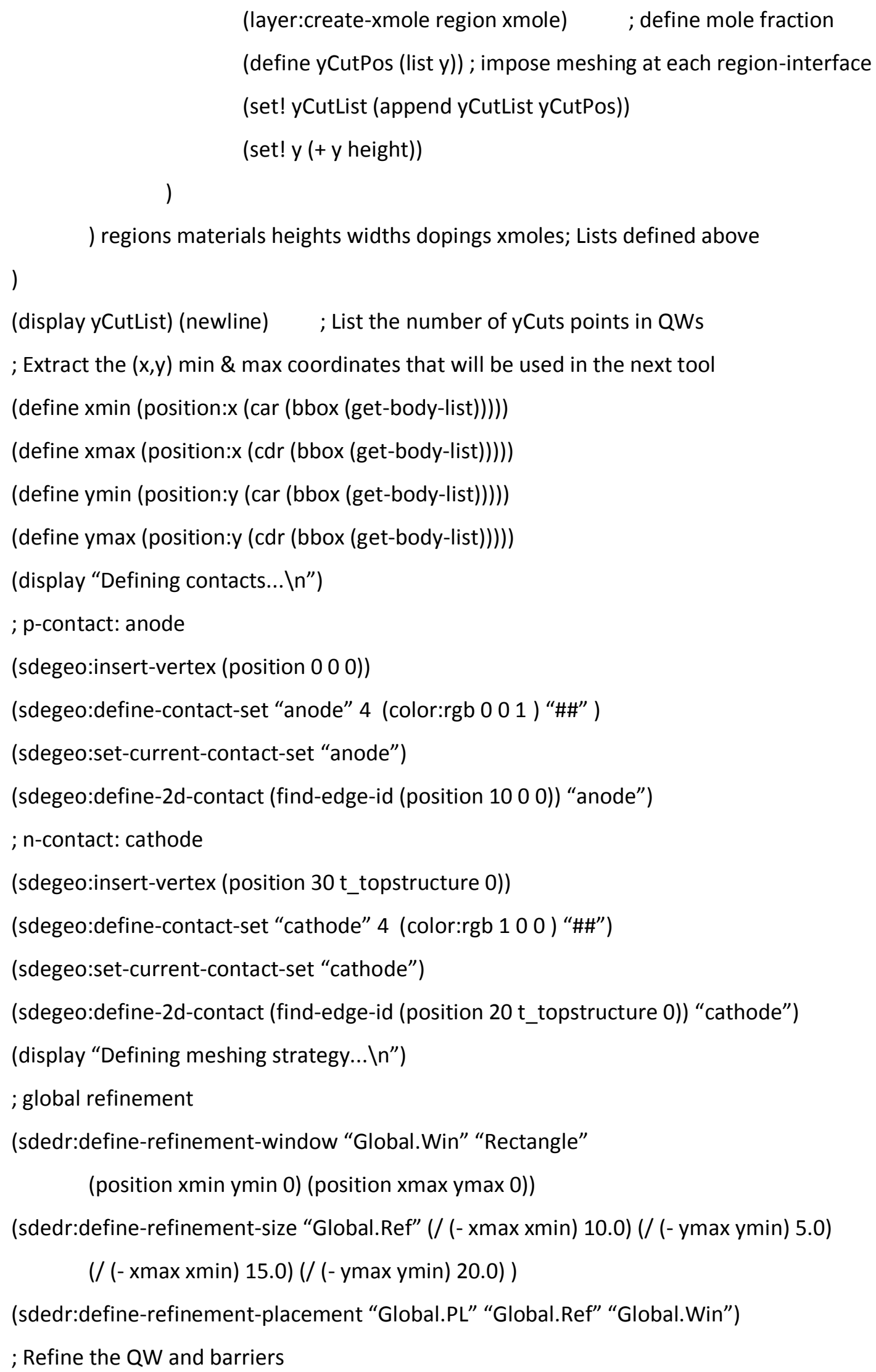


(sdedr:define-refinement-size "QW.Ref" (/ (- xmax xmin) 5.0) (/t_QW 3.9)

$$
\text { (/ (- xmax xmin) 10.0) (/t_QW 3.9)) }
$$

(sdedr:define-refinement-size "Barrier.Ref" (/ (- xmax xmin) 5.0) (/ t_B 7.9)

$$
\text { (/ (- xmax xmin) 10.0) (/t_B 7.9)) }
$$

(sdedr:define-refinement-region "RefPlace.QW" "QW.Ref” “QW")

(sdedr:define-refinement-region "RefPlace.Barrier0" "Barrier.Ref" "Barrier0")

(sdedr:define-refinement-region "RefPlace.Barrier1" "Barrier.Ref" "Barrier1")

(sdeaxisaligned:set-parameters "yCuts" yCutList)

(display “

(display “;;meshing electrical structure $\backslash n$ ”)

(display “

; Build electrical Mesh

(sde:build-mesh “snmesh” “” “n@node@_el_msh”)

(display "done. $(n$ ")

(display "SDE completed sucessfully. Have a nice day.")(newline)

\section{A.2 Sentaurus Device}

* sdevice_des.cmd

\#setdep@node|sde@

*

File \{

*-Input

$$
\text { Grid = "@tdr@" }
$$

Parameter = "@parameter@"

*-Output

$$
\text { Current = "@plot@" }
$$

Plot="@tdrdat@"

Output = "@log@"

\}

$*$

Electrode \{ 


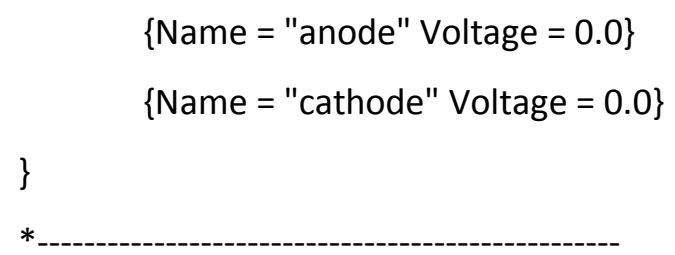

Physics \{

AreaFactor=@ @ 1e11/wtot $>@ *$ to get current in $\mathrm{mA} / \mathrm{cm}^{\wedge} 2$

Mobility ()

EffectivelntrinsicDensity (NoBandGapNarrowing)

Thermionic

Fermi

Heterolnterface

Optics (

ComplexRefractivelndex (WavelengthDep(Real Imag))

OpticalGeneration (

QuantumYield (StepFunction (Bandgap))

ComputeFromMonochromaticSource

) * end OpticalGeneration

Excitation (

Theta $=0 *$ Normal incidence

Polarization $=0.5 *$ Unpolarized light

Wavelength=@wstart@*[um]

Intensity=@intensity@*[W/cm2]

Window ("L1") (

Origin $=(0,0,0)$

XDirection $=(1,0,0)$

Line (

X1 =@wfront@

X2=@wtot@

)

)

) * end Excitation

OpticalSolver ( 
TMM (

NodesPerWavelength $=100 *$ Number of TMM mesh points

IntensityPattern = Envelope

LayerStackExtraction (

WindowName = "L1"

Position=(@<wtot $/ 2>@, 0,0)$

Mode $=$ RegionWise

)

) *end TMM

) * end OpticalSolver

) * end Optics

\}$^{*}$ end Physics

Physics (material = "GaN") \{Recombination (Radiative) $\}$

Physics (material = "InGaN") \{Recombination (Radiative)

Plot \{

*- Doping Profiles

Doping DonorConcentration AcceptorConcentration

*- Band structure

BandGap BandGapNarrowing ElectronAffinity

ConductionBandEnergy ValenceBandEnergy

eQuasiFermiEnergy hQuasiFermiEnergy

*- Carrier Densities

eDensity hDensity

EffectivelntrinsicDensity IntrinsicDensity

*- Fields, Potentials and Charge distributions

ElectricField/Vector

Potential

SpaceCharge

*- Currents

Current/Vector eCurrent/Vector hCurrent/Vector

CurrentPotential * for visualizing current lines

eMobility hMobility 
*-Generation/Recombination

TotalRecombination

eLifeTime hLifeTime

*- Optical Generation

ComplexRefractivelndex QuantumYield

OpticalIntensity AbsorbedPhotonDensity OpticalGeneration

\}

$*$

CurrentPlot \{

ModelParameter = "Wavelength"

OpticalGeneration(Integrate(Semiconductor) )

\}

Math \{

Digits $=6$

NoAutomaticCircuitContact

DirectCurrentComputation

Method $=$ Blocked

Submethod $=$ Pardiso

Derivatives

Notdamped $=100$

Iterations $=15$

RelErrControl

ErReff(electron) $=1 \mathrm{e} 2$

$\operatorname{ErReff}($ hole $)=1 \mathrm{e} 2$

ElementEdgeCurrent

* -CheckUndefinedModels

ExtendedPrecision

DualGridInterpolation (Method $=$ Simple)

* Number_Of_Threads $=4$

CurrentPlot (IntegrationUnit $=\mathrm{cm}$ )

\}

Solve\{ 


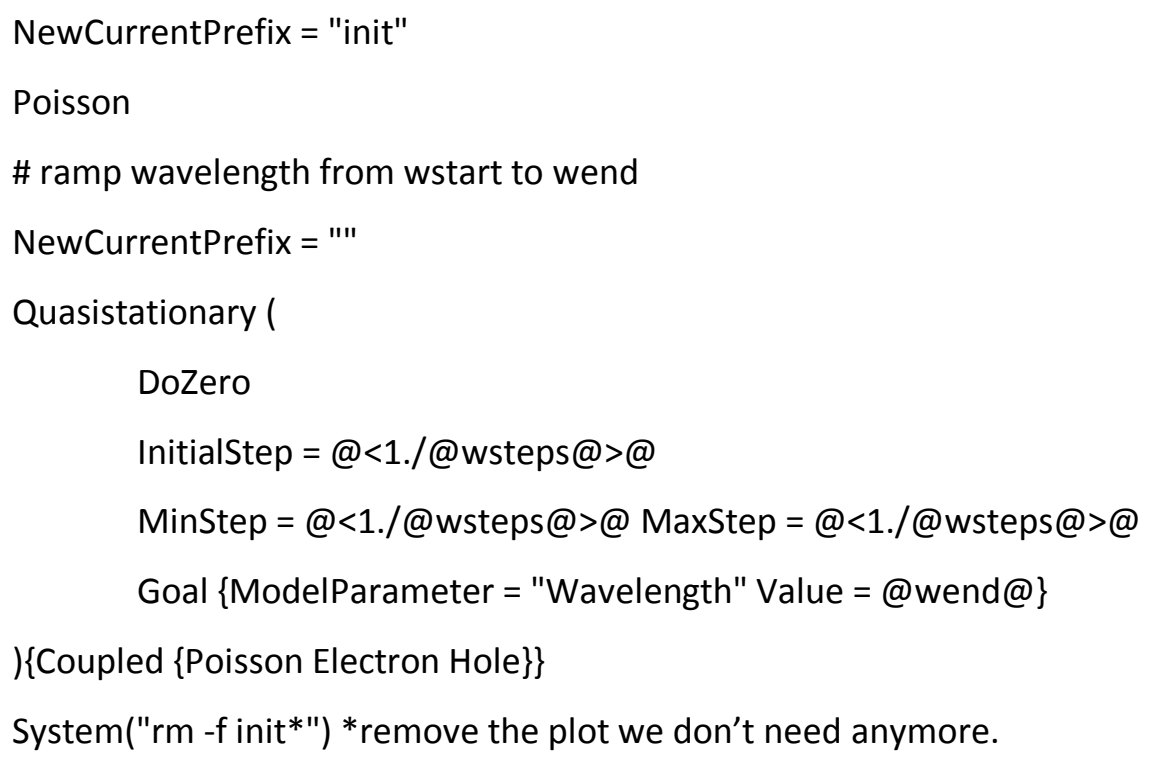

\section{A.3 Parameter Files}

\section{A.3.1 Barrier: GaN}

* GaN.par

* TRANSPORT

Epsilon

\{

* Ratio of the permittivities of material and vacuum

* epsilon() = epsilon

epsilon $=8.3$

\}

Bandgap

\{

* Eg = Eg0 + alpha Tpar2 / (beta + Tpar) - alpha T2 / (beta + T)

* Parameter 'Tpar' specifies the value of lattice

* temperature, at which parameters below are defined

* Chi0 is electron affinity.

$$
\begin{array}{ll}
\text { ChiO }=3.4 & \#[\mathrm{eV}] \\
\text { Bgn2Chi }=0.5 & \#[1]
\end{array}
$$




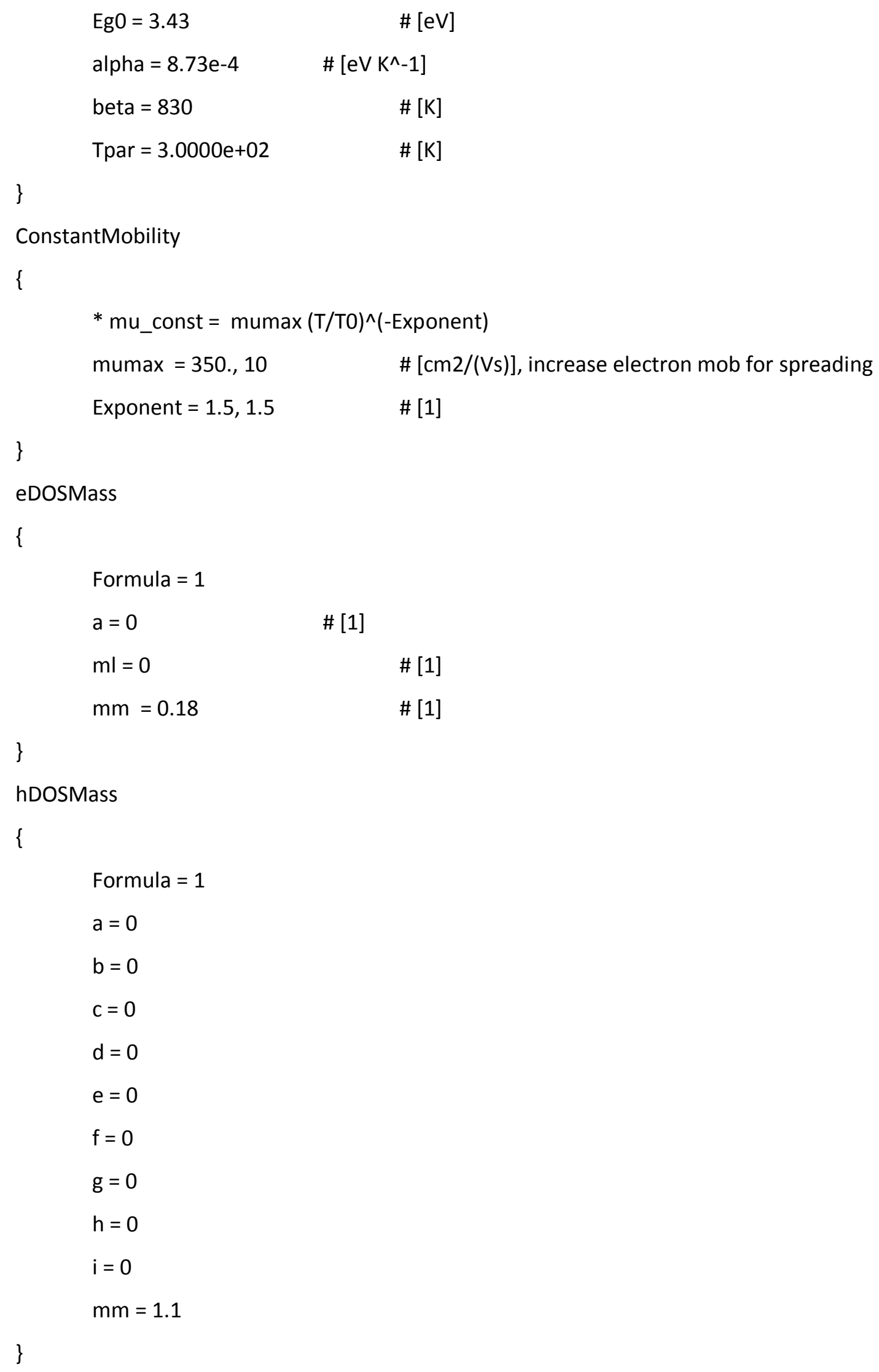


Ionization

\{

$$
\begin{array}{lc}
\text { E_P_0 }=0.02 & \#[\mathrm{eV}] \\
\text { alpha_P }=3.1000 \mathrm{e}-08 & \#[\mathrm{eV} \mathrm{cm}] \\
\text { g_P }=2 & \#[1] \\
\text { Xsec_P }=1.0000 \mathrm{e}-12 & \#\left[\mathrm{~cm}^{\wedge} 2 / \mathrm{sec}\right. \\
\text { E_B_0 }=0.199 & \#[\mathrm{eV}] \\
\text { alpha_B }=3.1000 \mathrm{e}-08 & \#[\mathrm{eV} \mathrm{cm}] \\
\text { g_B }=4 & \quad \#[1] \\
\text { Xsec_B }=1.0000 \mathrm{e}-12 & \#\left[\mathrm{~cm}{ }^{\wedge} 2 / \mathrm{sec}\right] \\
\text { NdCrit }=1.0000 \mathrm{e}+22 & \#[\mathrm{~cm}-3] \\
\text { NaCrit }=1.0000 \mathrm{e}+22 & \#[\mathrm{~cm}-3] \\
&
\end{array}
$$


refractiveindex $=2.45$
alpha $=0.0$
$\#[1 / K]$
Tpar $=300$.
\# [K]

\}

ComplexRefractivelndex

\{

* Complex refractive index model: $n \_c o m p l e x=n+i * k$ (unitless)

* with $n=n \_0+$ delta_n_lambda + delta_n_T + delta_n_carr + delta_n_gain

* k = k_0 + delta_k_carr

* Mole fraction dependence (real and imag):

*n_0, k_0

$\#$ n_0 $=2.45$

$\#$ k_0 $=0.0$

$\#$ k_0 $=0.01$

* Wavelength dependence (real and imag)

* Formula 0: delta_n_lambda $=$ Cn_lambda $*$ lambda + Dn_lambda $*$ lambda^2

* delta_k_lambda =Ck_lambda *lambda + Dk_lambda * lambda^2

* Formula 1: Read tabulated values

* NumericalTable (...)

* Formula 2: Read tabulated values from file

* Numerical table $=\langle$ string $>$

Formula $=1$

NumericalTable(

$\begin{array}{lll}0.30995 & 2.746248 & 0.36 ; \\ 0.354228571 & 2.599 & 0.32 ; \\ 0.413266667 & 2.492269 & 0.016 ; \\ 0.433928335 & 2.467332 & 0.012 ; \\ 0.45459 & 2.446464 & 0.008 ; \\ 0.49592 & 2.413675 & 0 ; \\ 0.6199 & 2.355923 & 0 ; \\ 0.826533333 & 2.314508 & 0 ; \\ 1.2398 & 2.286603 & 0 ;\end{array}$


)

* Temperature dependence (real):

*delta_n_T $=$ n_0 $*\left(C n \_t e m p *(T-T p a r)\right)$

Cn_temp $=4.273 \mathrm{e}-5 \quad \#\left[\mathrm{~K}^{\wedge}-1\right]$

Tpar $=3.0000 \mathrm{e}+02 \quad$ \#[K]

* Carrier dependence (real)

*delta_n_carr $=-$ Cn_carr * (const.) $*\left(n / m \_e+p / m \_h\right)$

Cn_carr $(0)=0$

Cn_carr(1) = 0

* Carrier dependence (imag)

* delta_k_carr $=$ wavelength $/\left(4^{*} \mathrm{PI}\right) *\left(C k \_c a r r \_n * n+C k \_c a r r \_p * p\right)$

Ck_carr $=0.0000 \mathrm{e}+00,0.0000 \mathrm{e}+00 \quad \#[1]$

* Gain dependence (real)

* lin: delta_k_gain $=C n \_g a i n *((n+p) / 2-N p a r)$

* log: delta_k_gain $=C n \_g a i n * \log ((n+p) /(2-N p a r))$

Cn_gain $=0.0000 \mathrm{e}+00 \quad \#\left[\mathrm{~cm}^{\wedge} 3\right]$

Npar $=1.0000 \mathrm{e}+18 \quad \#\left[\mathrm{~cm}^{\wedge}-3\right]$

\}

SchroedingerParameters \{

* For the hole masses for Schroedinger equation different formulas

* can be used.

* formula $=1$ (for materials with Si-like hole band structure)

${ }^{*} \mathrm{~m}(\mathrm{k}) / \mathrm{m0}=1 /\left(A+-\operatorname{sqrt}\left(B+C^{*}\left((x y)^{\wedge} 2+(y z)^{\wedge} 2+(z x)^{\wedge} 2\right)\right)\right)$

* where $\mathrm{k}=(\mathrm{x}, \mathrm{y}, \mathrm{z})$ is unit normal vector in reziprocal

* space. '+' for light hole band, '-' for heavy hole band

* formula $=2$ : Heavy hole mass $\mathrm{mh}$ and light hole mass $\mathrm{ml}$ are

* specified explicitly.

* formula $=3: \mathrm{A}$ is the relative effective mass. This

* formula must only be used for metal and will used in

* tunnelling current computation only.

* formula<0 means no default model and no default parameters

* are available, so you have to provide values for 
* 'formula' and the respective parameters in order to use

* this parameter set.

* In GaN the valence band ordering from the top is: hh, lh and cr

* The valance band effective masses are anisotropic with different

* values along the c-axis and the plane perpendicular to the c-axis.

* Most nitride structues are grown along the c-axis, with the QWs

* perpendicular to this axis. Thus the quntization direction is

* parallel to the c-axis, and the c-axis mass should be used in the

* schrodinger solver.

* Formula 2 parameters:

formula $=2$

$\mathrm{ml}=0.5$

$\mathrm{mh}=1.3$

* Lifting of degeneracy of bulk valleys. The value for

* electrons is added to the band edge for the subband

* ladder of lower degeneracy if positive, and subtracted

* from the band edge for the ladder of higher degeneracy

* if negative. (that is, the value of the band edge is

* always increased). For holes, the value is subtracted from

* the band edge for the heavy hole band is positive,

* add added tp that of the light hole band if

* negative. The signs are such that the shift always

* moves the band edges 'outward', away from midgap. The

* gap itself is defined as the separation of the

* unshifted band edges and remains unaffected.

offset $=0.0000 \mathrm{e}+00,0.0000 \mathrm{e}+00 \quad \#[\mathrm{eV}]$

\}

* HEAT

Kappa

\{

* Lattice thermal conductivity

* Formula = 1: 


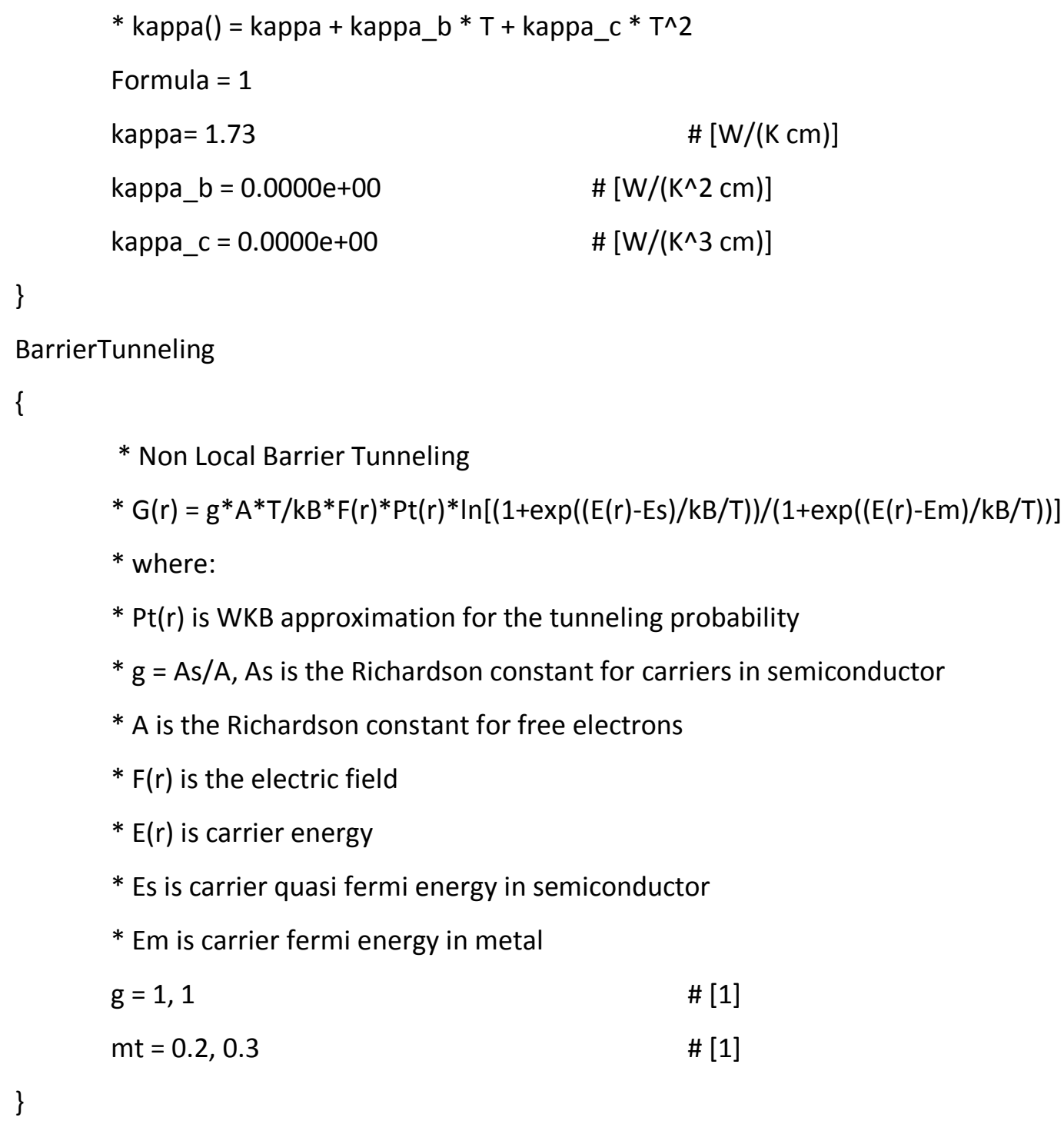

\section{A.3.2 Quantum Well: InGaN}

* InGaN.par

* Material parameter file for $\ln (x) G a(1-x) N$

$*_{\text {for } x}=0.16$

* TRANSPORT

Epsilon \{

epsilon $=9.436$

\}

Bandgap $\left\{{ }^{*} \mathrm{Eg}=\right.$ Eg0+alpha*Tpar^2/(beta + Tpar)-alpha*T^2/(beta+T) Chi0 $=3.955968$ 


$$
\begin{aligned}
& \text { Eg0 }=2.63576 \\
& \text { alpha }=1.024 \mathrm{e}-3 \\
& \text { beta }=1247.4857 \\
& \text { Tpar }=300.0 \\
& \quad
\end{aligned}
$$




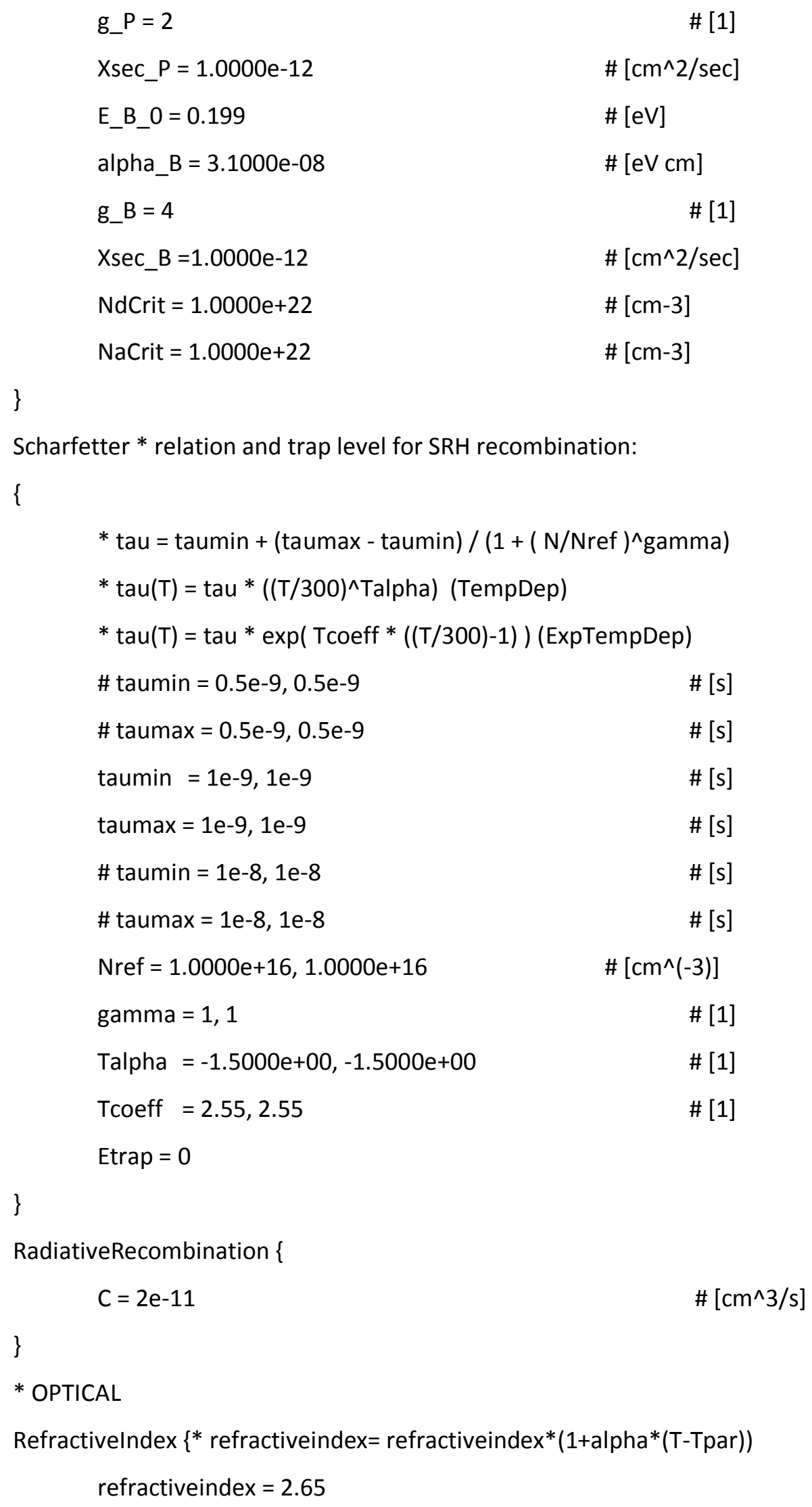



alpha $=0.0$
$\#[1 / K]$
Tpar $=300$.
$\#[\mathrm{~K}]$

\}

\section{ComplexRefractivelndex}

\{

* Complex refractive index model: $n \_c o m p l e x=n+i^{*} k$ (unitless)

* with $\mathrm{n}=\mathrm{n} \_0+$ delta_n_lambda + delta_n_T + delta_n_carr + delta_n_gain

* k $=$ k_0 + delta_k_carr

* Mole fraction dependence (real and imag)

* n_0, k_0

n_0 $=2.65$

k_0 $=0.0$

* Wavelength dependence (real and imag)

* Formula 0: delta_n_lambda $=$ Cn_lambda $*$ lambda + Dn_lambda $*$ lambda^2

* delta_k_lambda $=$ Ck_lambda * lambda + Dk_lambda $*$ lambda^2

* Formula 1: Read tabulated values

* NumericalTable (...)

* Formula 2: Read tabulated values from file

* Numerical table $=<$ string $>$

Formula $=0$

* Temperature dependence (real):

*delta_n_T $=$ n_0 $*\left(C n \_t e m p *(T-T p a r)\right)$

Cn_temp $=0.0$

Tpar $=3.0000 \mathrm{e}+02$ $\#[K]$

* Carrier dependence (real)

* delta_n_carr $=-$ Cn_carr $*($ const. $) *\left(n / m \_e+p / m \_h\right)$

Cn_carr $(0)=0$

Cn_carr(1) $=0$ \#[1]

* Carrier dependence (imag)

* delta_k_carr $=$ wavelength $/(4 * \mathrm{PI}) *\left(C k \_c a r r \_n * n+C k \_c a r r \_p * p\right)$

Ck_carr $=0.0000 \mathrm{e}+00,0.0000 \mathrm{e}+00$

\# [1]

* Gain dependence (real) 
* lin: delta_k_gain $=$ Cn_gain * $((n+p) / 2-N p a r)$

* log: delta_k_gain $=C n \_g a i n * \log ((n+p) /(2-N p a r))$

$$
\begin{array}{ll}
\text { Cn_gain }=0.0000 \mathrm{e}+00 & \#\left[\mathrm{~cm}^{\wedge} 3\right] \\
\text { Npar }=1.0000 \mathrm{e}+18 & \#\left[\mathrm{~cm}^{\wedge}-3\right]
\end{array}
$$

\}

SchroedingerParameters \{

* In GaN the valence band ordering from the top is: hh, lh and cr.

* In InN the valence band ordering from the top is: hh, Ih and cr.

* The valance band effective masses are anisotropic with different

* values along the c-axis and the plane perpendicular to the c-axis.

* Most nitride structues are grown along the c-axis, with the QWs

* perpendicular to this axis. Thus the quantization direction is

* parallel to the c-axis, and the c-axis mass should be used in the

* schrodinger solver.

* For top most effective mass interpolate between GaN heavy hole and InN

* heavy hole mass. For next hole band interpolate between GaN

* light hole and InN light hole mass.

* Formula 2 parameters:

formula $=2$

$\mathrm{mh}=1.348$

$\mathrm{ml}=0.4376$

\}

* HEAT

Kappa \{

Formula $=1$

* Formula $=1$ :

* kappa ()$=$ kappa + kappa_b $* T+$ kappa_c $* T^{\wedge} 2$

kappa $=0.756857142857$

\# $[\mathrm{W} /(\mathrm{K} \mathrm{cm})]$

kappa_b $=0.0000 \mathrm{e}+00 \quad$ \#[W/(K^2 cm)

kappa_c $=0.0000 \mathrm{e}+00 \quad \#\left[\mathrm{~W} /\left(\mathrm{K}^{\wedge} 3 \mathrm{~cm}\right)\right]$

\}

BarrierTunneling 
* Non Local Barrier Tunneling

* $G(r)=g^{*} A^{*} T / k B * F(r) * P t(r) * \ln [(1+\exp ((E(r)-E s) / k B / T)) /(1+\exp ((E(r)-E m) / k B / T))]$

* where:

* $\mathrm{Pt}(\mathrm{r})$ is WKB approximation for the tunneling probability

* $\mathrm{g}=\mathrm{As} / \mathrm{A}, \mathrm{As}$ is the Richardson constant for carriers in semiconductor

* $A$ is the Richardson constant for free electrons

* $F(r)$ is the electric field

* $E(r)$ is carrier energy

* Es is carrier quasi fermi energy in semiconductor

* Em is carrier fermi energy in metal

$g=1,1$

\# [1]

$\mathrm{mt}=0.1856,0.2952$

\# [1]

\} 


\section{Appendix B}

\section{Study of quantum properties of a single GaN/InGaN QW using tiberCAD tool}

\section{B.1 GMSH geometry file}

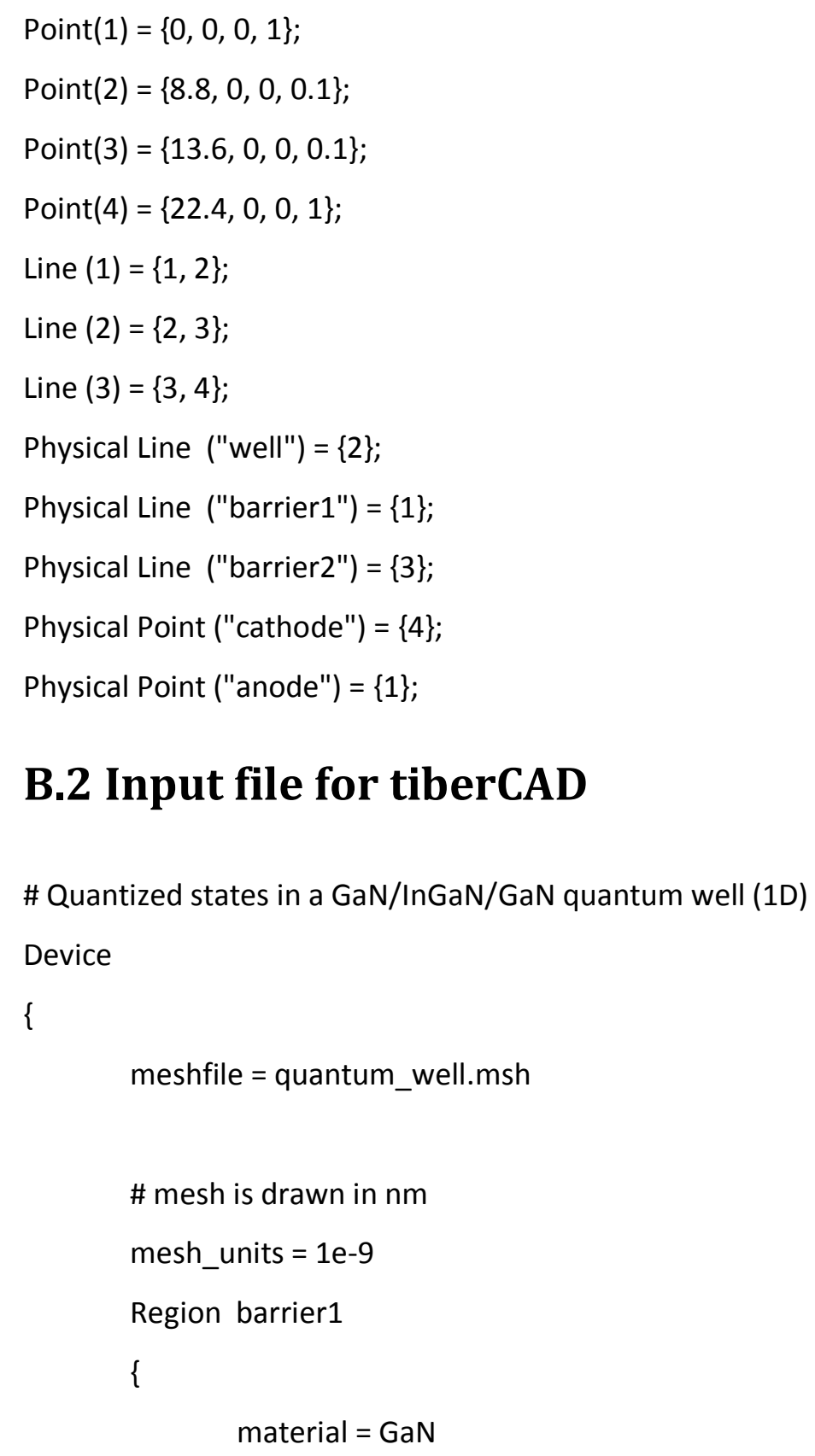

\section{B.2 Input file for tiberCAD}




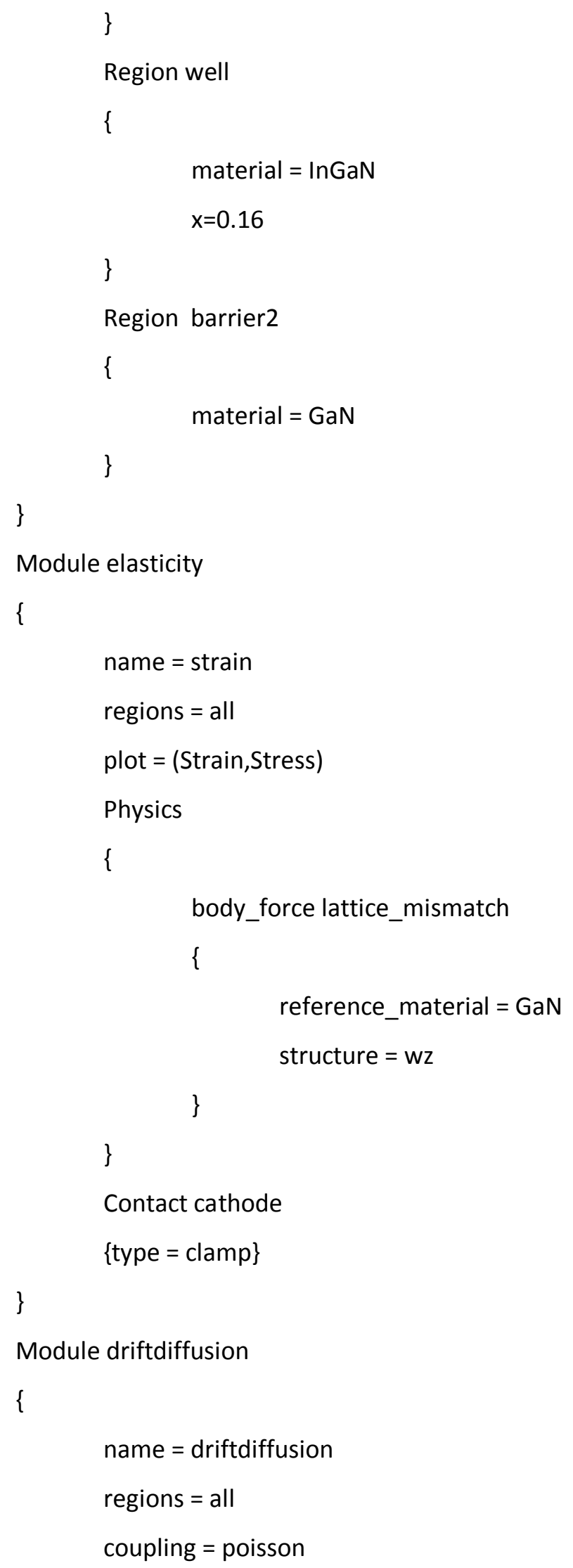




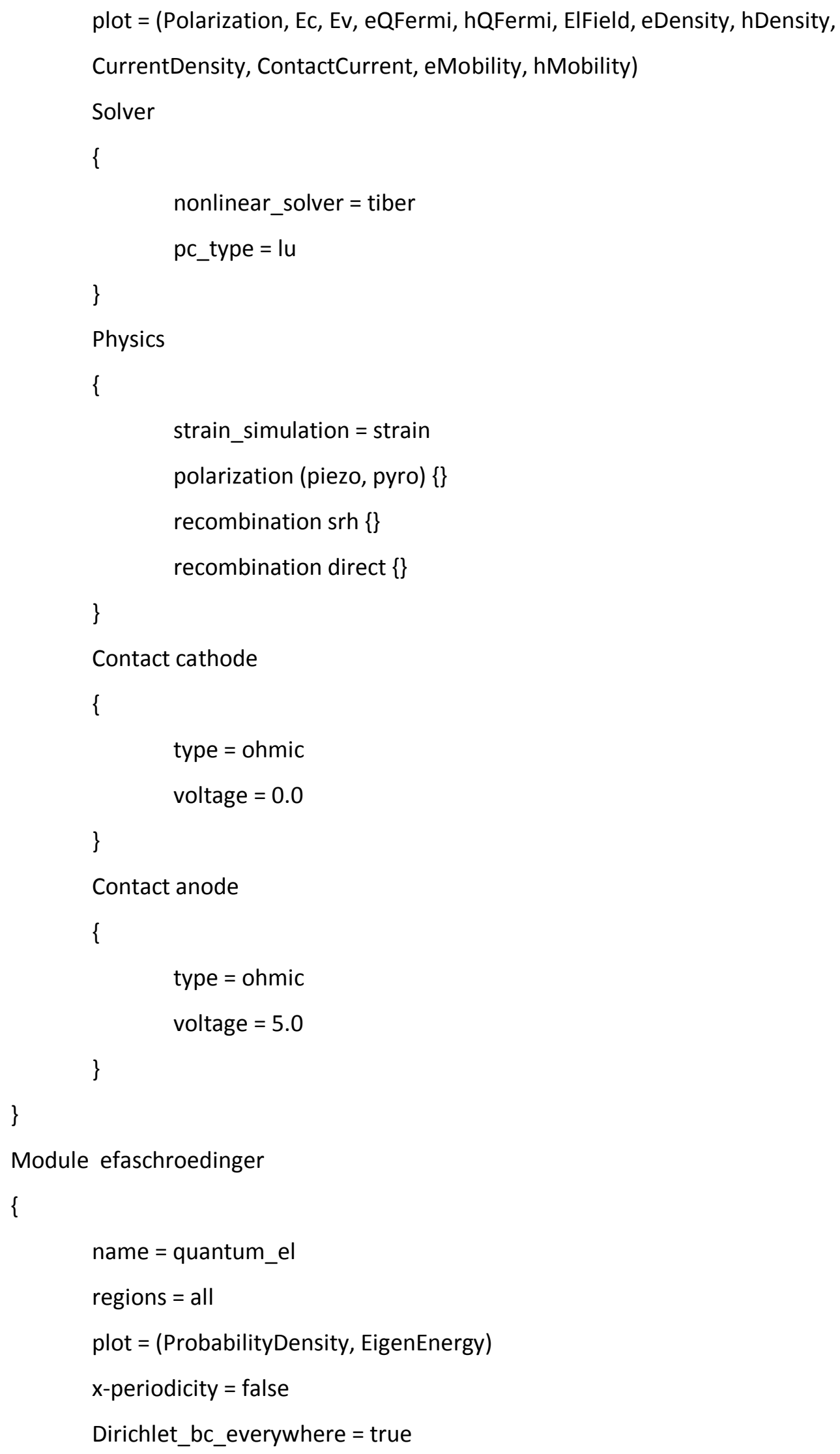




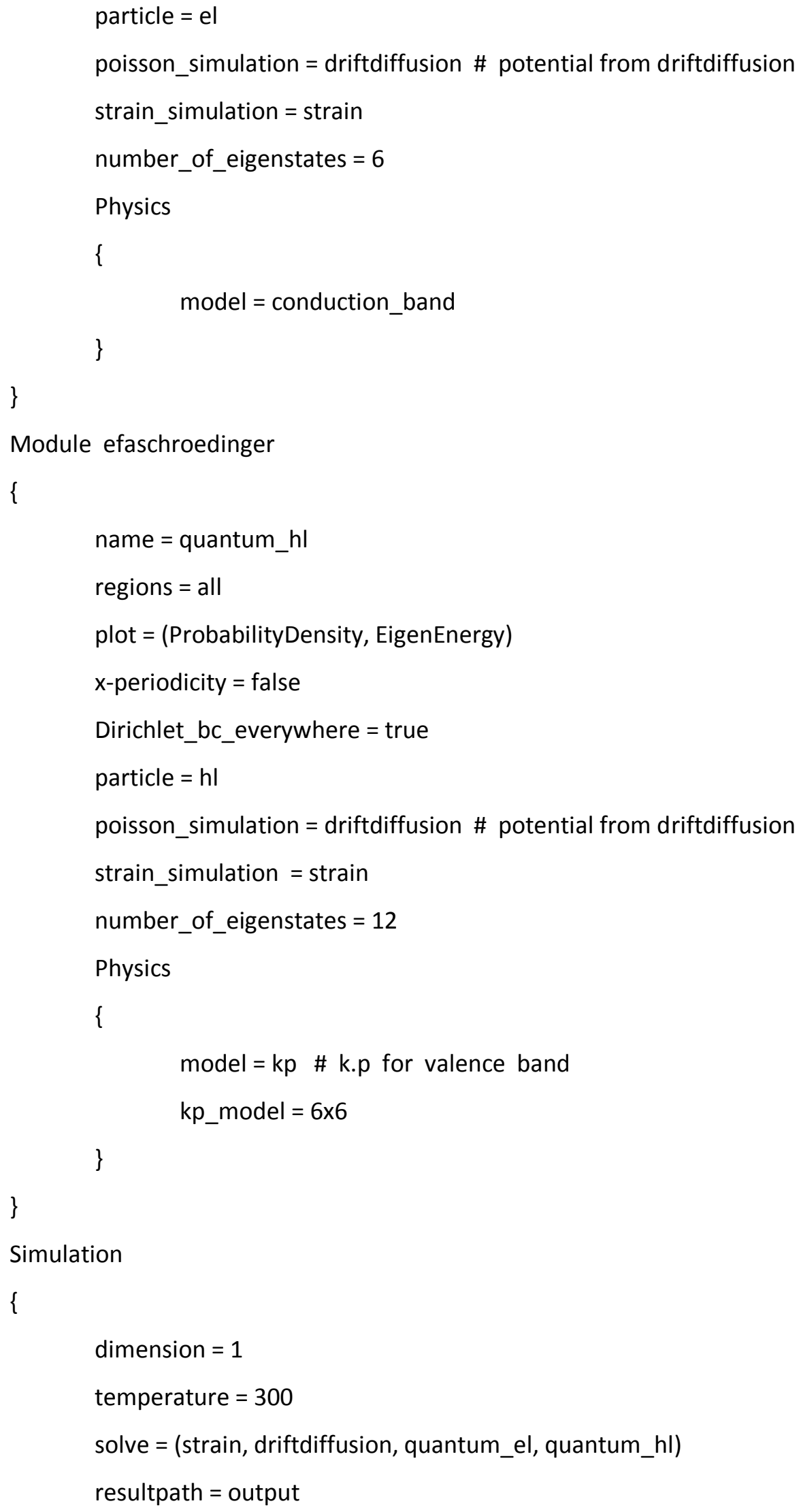


output_format = grace

\}

\section{B.3 Material Files}

\section{B.3.1 GaN}

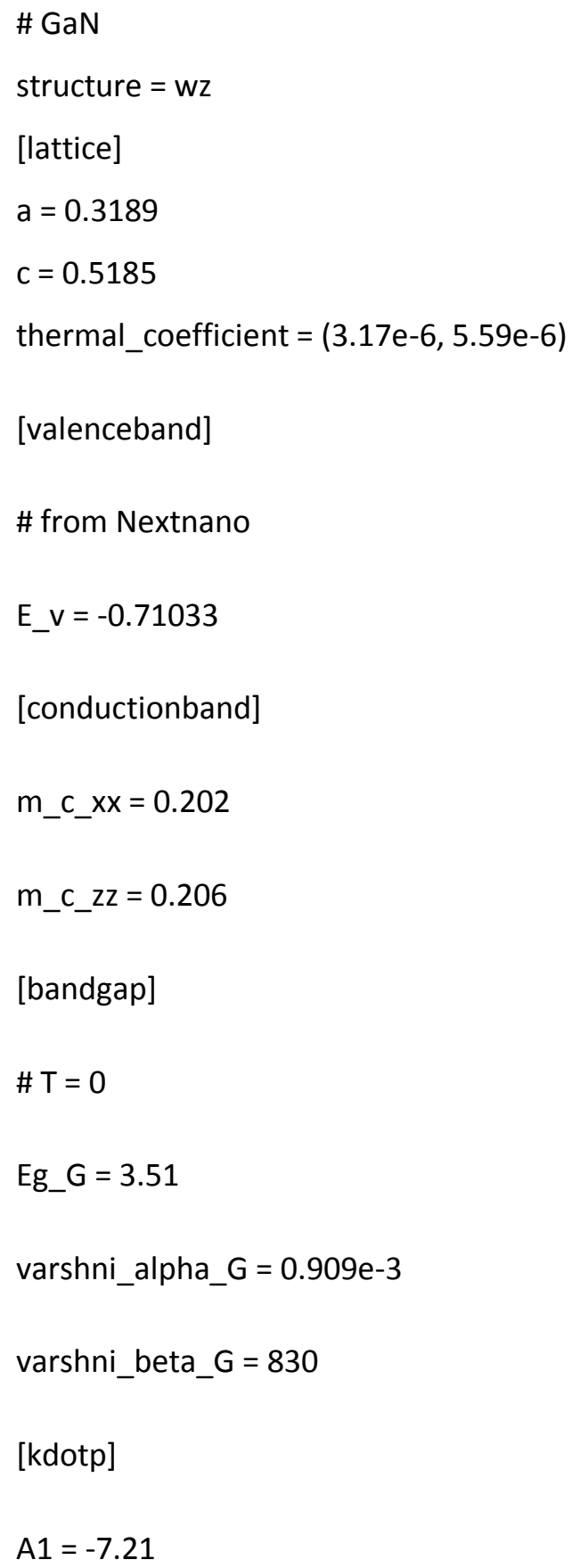




$$
\begin{aligned}
& A 2=-0.44 \\
& A 3=6.68 \\
& A 4=-3.46 \\
& A 5=-3.4 \\
& A 6=-4.9 \\
& \text { delta_cr }=0.01 \\
& \text { delta_s }=0.017 \\
& \text { [deformation_potentials] } \\
& a \_x=-6.80 \\
& a_{-} z=-8.60 \\
& D 1=-3.7 \\
& \mathrm{D} 2=4.5 \\
& \text { D3 }=8.2 \\
& \text { D4 }=-4.1 \\
& \mathrm{D} 5=-4.0 \\
& \text { D6 }=-5.5 \\
& \text { [permittivity] } \\
& \text { \# static dielectric constant } \\
& \text { permittivity }=(10.01,10.01) \\
& \text { optical_epsilon_x = } 7.3 \\
& \text { optical_epsilon_z }=7.3
\end{aligned}
$$




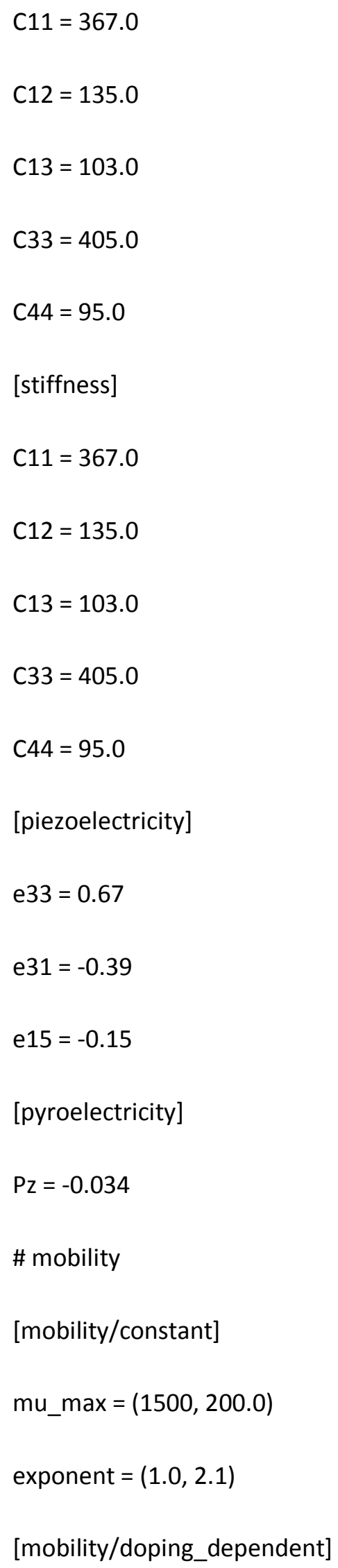




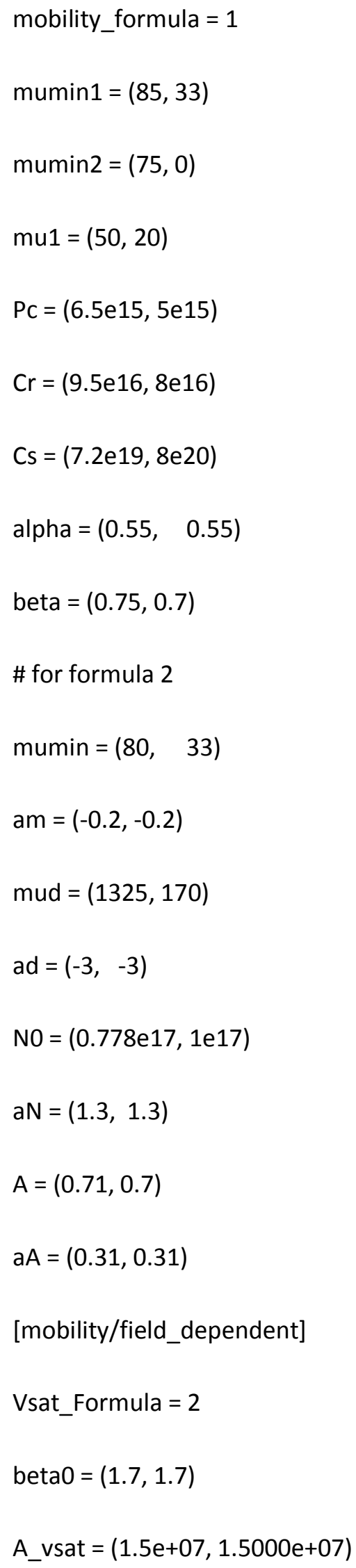




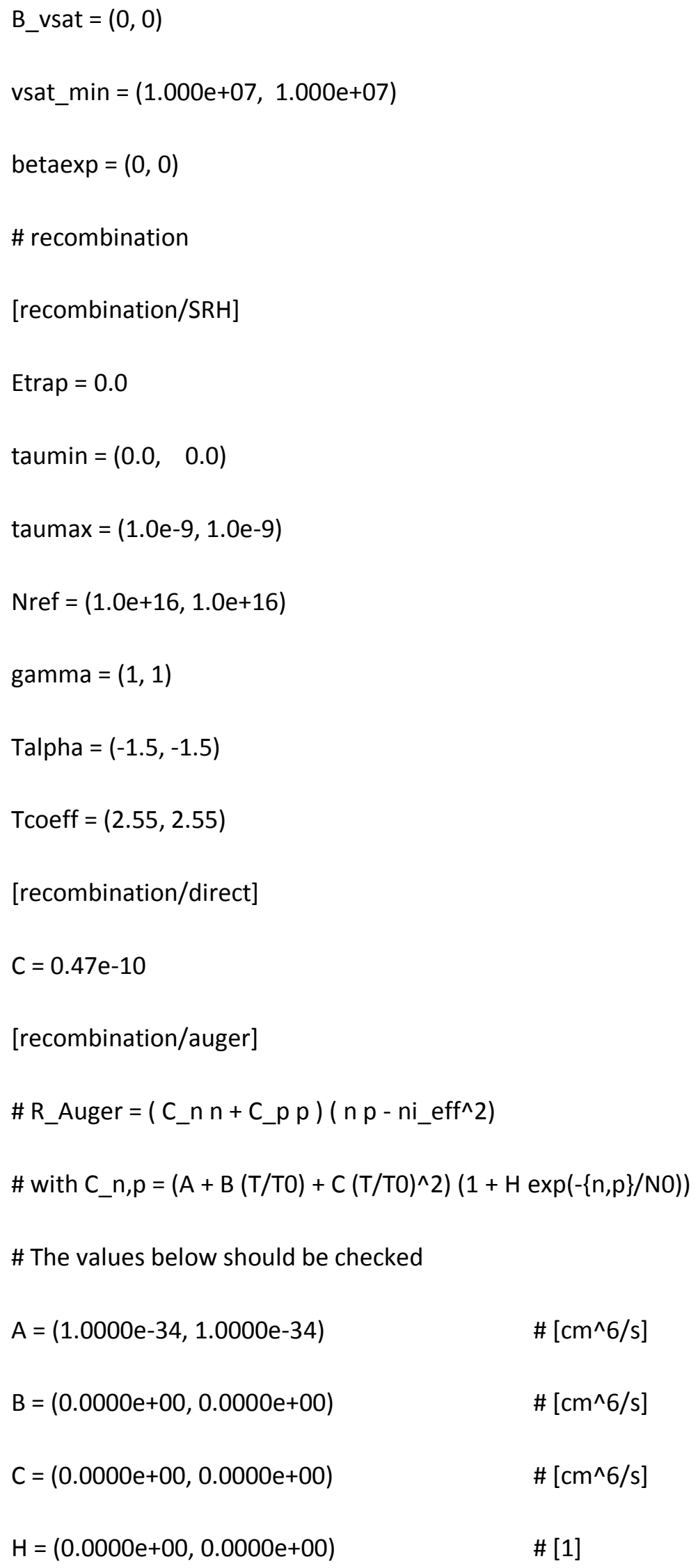




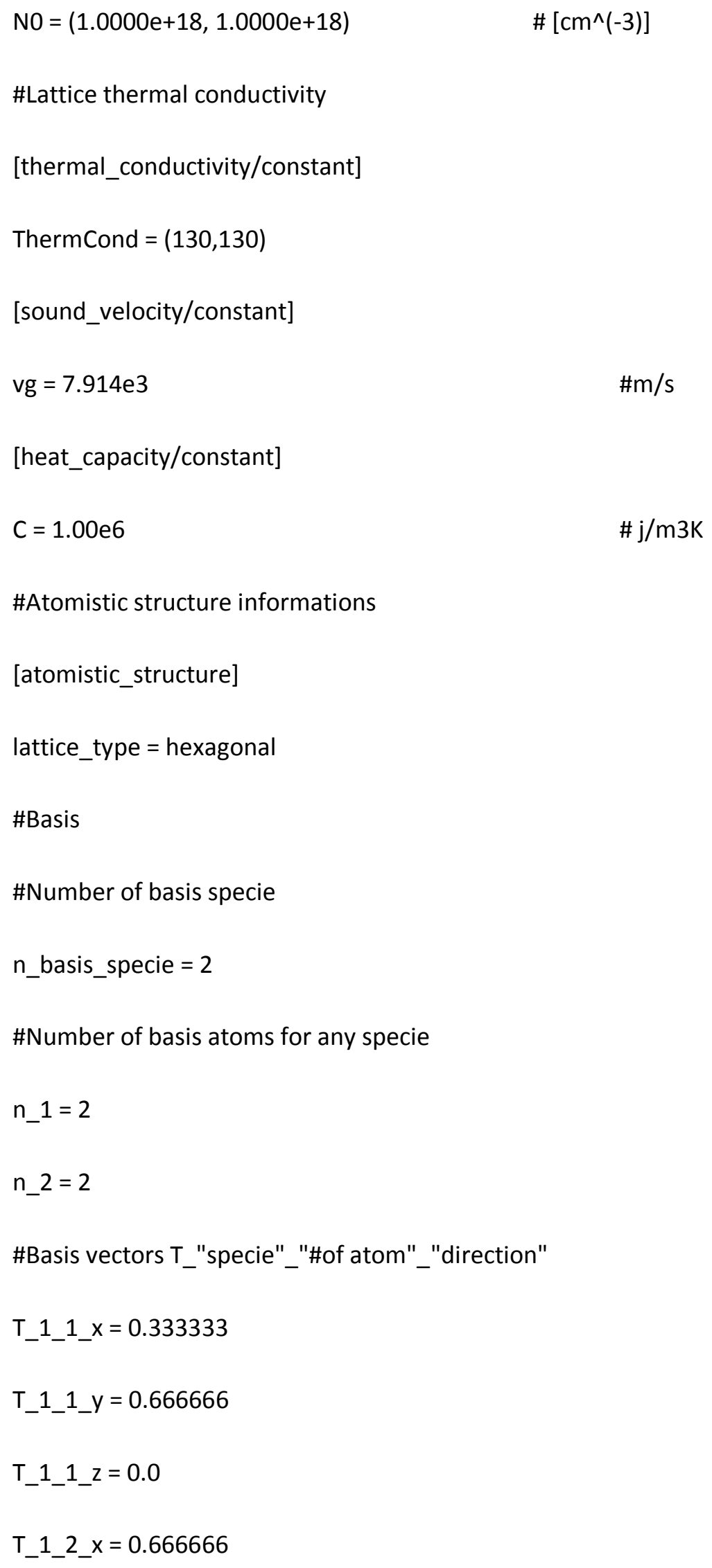




$$
\begin{aligned}
& \text { T_1_2_y }=0.333333 \\
& \text { T_1_2_z }=0.5 \\
& \text { T_2_1_x }=0.333333 \\
& \text { T_2_1_y }=0.666666 \\
& \text { T_2_1_z }=0.375000 \\
& \text { T_2_2_x }=0.666666 \\
& \text { T_2_2_y }=0.333333 \\
& \text { T_2_2_z }=0.875000 \\
& \text { \#Name of species } \\
& \text { specie_1 = Ga } \\
& \text { specie_2 }=\mathrm{N} \\
& \text { [densityfunctional_tb] } \\
& \text { dataset }=\text { nitrides } \\
& \text { B.3.1 InGaN } \\
& \text { \# InGaN } \\
& \text { alloy }=2 \\
& \text { structure }=\mathrm{wz} \\
& \text { comp_A }=\ln N \\
& \text { comp_B }=\mathrm{GaN} \\
& \text { [bandgap] } \\
& \text { bow_Eg_G }=1.4
\end{aligned}
$$


bow_Eg_X $=0.69$

bow_Eg_L $=1.84$

[pyroelectricity]

bow_Pz $=-0.037$

\#Atomistic structure informations

[atomistic_structure]

n_basis_specie $=2$

specie_1 $=$ In

specie_2 $=\mathrm{N}$

[densityfunctional_tb] 


\section{Appendix C}

\section{Study of influence of quantum wells on LED characteristics using Crosslight APSYS software}

\section{C.1 Device Structure}

\section{C.1.1 Layer File}

$\$$

begin_layer

$\$$

independent_mqw

column column_num $=1 \mathrm{w}=200$ mesh_num $=10 \mathrm{r}=0.85$

column column_num=2 $\mathrm{w}=100$ mesh_num=8 $r=1.15$

top_contact column_num $=1$ from $=0.0$ to $=50$ contact_num $=2$

top_contact column_num $=2$ from $=30$ to $=100$ contact_num $=1$

\$ substrate

layer_mater macro_name=sapphire column_num $=1$

layer_mater macro_name=sapphire column_num $=2$

layer $d=430 \quad n=8 \quad r=0.7$

$\$$ AIN layer

layer_mater macro_name=algan var1=1 column_num=1 var_symbol1=x

layer_mater macro_name=algan var $1=1$ column_num $=2$ var_symbol $1=x$

layer $d=0.03 n=8 \quad r=0.8$

$\$$ unGaN layer

layer_mater macro_name=algan var1 $1=0$ column_num $=1$ var_symbol1 $1=x$

layer_mater macro_name=algan var1 $1=0$ column_num $=2$ var_symbol $1=x$

$\$ n G a N$ layer

layer_mater macro_name=algan var1=0 column_num $=1$ var_symbol1=x n_doping $=1 \mathrm{e} 24$ 
layer_mater macro_name=algan var1=0 column_num $=2$ var_symbol1=x n_doping $=1 \mathrm{e} 24$

layer $d=1.1 \quad n=8 \quad r=0.8$

layer_mater macro_name=algan var1=0 column_num $=1$ var_symbol1=x n_doping $=1 \mathrm{e} 24$

layer_mater macro_name=void column_num $=2$

layer $d=0.4 n=5 \quad r=0.8$

$\$ M Q W$ region

include file=ganled.bar

include file=ganled.qw

include file=ganled.bar

include file=ganled.qw

include file=ganled.bar

include file=ganled.qw

include file=ganled.bar

include file=ganled.qw

include file=ganled.bar

include file=ganled.qw

include file=ganled.bar

$\$$ pAIGaN layer

layer_mater macro_name=algan var1 $=0.1$ column_num $=1$ var_symbol1 $=x$ p_doping $=3 \mathrm{e} 23$

layer_mater macro_name=void column_num $=2$

layer $d=0.01 \mathrm{n}=6 \mathrm{r}=1$

$\$$ pGaN layer

layer_mater macro_name=algan var1=0 column_num $=1$ var_symbol1=x p_doping $=3 e 23$

layer_mater macro_name=void column_num=2

layer $d=0.14 n=4 \quad r=1$

$\$$

end_layer 


\section{C.1.2 Barrier file}

\$ GaN

layer_mater macro_name=ingan var $1=0.0$ column_num $=1$ var_symbol $1=x$

layer_mater macro_name=void column_num =2

layer $\mathrm{d}=0.0088 \mathrm{n}=12 \mathrm{r}=-1.4$

\section{C.1.3 QW file}

$\$ \ln G a N$

layer_mater macro_name=ingan var $1=0.16$ column_num $=1 \& \&$

active_macro=InGaN/InGaN avar1=0.16 avar2 $=0 \& \&$

avar_symbol1=xw avar_symbol $2=x b$ var_symbol $1=x$

layer_mater macro_name=void column_num $=2$ active_macro=void

layer $\mathrm{d}=0.0048 \mathrm{n}=8 \mathrm{r}=-1.3$

\section{C.2 Setting up a Simulation}

$\$$

begin

load_mesh mesh_inf=ganled.msh

output sol_outf=ganled.out

more_output qw_states=yes

include file=ganled.doping

include file=ganled. mater

polarization_charge_model screening $=0.5$ vector $=\left(\begin{array}{lll}0 & 1 & 0\end{array}\right)$

set_active_reg tau_scat $=0.4 \mathrm{e}-13$

modify_qw tail_energy $=0.03$

\$self-consistent is necessary for polarization

self_consistent wave_range $=0.005$

$\$$ Quantum transport model helps deal with thin deep wells

q_transport

$\$$. mater shows superlattice is mater $=9$

mobility_xy dir=y factor_elec $=0.2$ mater $=9$

thermal_kappa_xy dir=y factor $=0.1$ mater=9 
$\$$ n-layer mobility may be enhanced due to SL design

max_electron_mob value $=1$ mater $=2$

min_electron_mob value $=1$ mater $=2$

\$ Isothermal temperature at equilibrium

temperature tem $\mathrm{p}=300$

\$ Turn on self-heating model

heat_flow damping_step $=1$

$\$$ thermal_cond at contact here will determine self-heating

contact num $=2$ type=ohmic thermal_type $=3 \& \&$

thermal_cond $=200$ extern_temp $=300$

$\$$----- initialize optical constants------

set_wavelength wavelength $=0.40$ backg_loss $=2000$

\$ Set LED model to "simple"

led_simple wavelength $=0.40$ spectrum_num $=50$

$\$$ Export raytracing data: convert 2D electrical simulation into

$\$ 3 D$ raytracing boxes

export_raytrace ray3d_convert=yes

\$ start solving

newton_par damping_step $=5$. max_iter $=100$ print_flag=3

equilibrium

newton_par damping_step $=1$. print_flag=3

scan var=voltage_1 value_to $=-10$ init_step $=0.1$ max_step $=0.5$ \&\&

auto_finish=current_1 auto_until=1.0 auto_condition=above

scan var=current_1 value_to $=60$ print_step $=20 \& \&$

init_step=1 min_step=1e-3 max_step=5

end 


\section{Bibliography}

[1] J. H. Burroughes, D. D. C. Bradley, A. R. Brown, R. N. Marks, K. Mackay, R. H. Friend, P. L. Burns, and A. B. Holmes, "Light-emitting diodes based on conjugated polymors," Nature, pp. 539 - 541, 1990.

[2] Optische Messetechnik, Handbook of LED Metrology, Instrument Systems.

[3] NIOSH, "National Institute for Occupational Safety and Health," 2012.

[4] Thermos, "LED Technology," 2007.

[5] A. E. Roggio, "NASA and other researchers test LEDs for space crops," 2010.

[6] Philips, "LED Lighting Applications".

[7] Sun X, Li D, Song H, Chen Y, Jiang H, Miao G, Li Z, "Short-wavelength light beam in situ monitoring growth of InGaN/GaN green LEDs by MOCVD," Nanoscale Res Lett, 2012.

[8] Rainer Minixhofer, "Integrating Technology Simulation into the Semiconductor Manufacturing Environment," Institute for Microelectronics, Vienna University of Technology, 2006.

[9] Luciano Lavagno, Grant Martin, and Louis Scheffer, Electronic Design Automation for Integrated Circuits, CRC Press , 2006.

[10] Safa Kasap and Peter Capper, Springer Handbook of Electronic and Photonic Materials, 2006.

[11] E. Fred Schubert, Light-Emitting Diodes, 2nd edition 2006.

[12] cree, "LED Lightning".

[13] W. S. Lee, "Polarization Engineering in GaN-based Light Emitting Diodes," Rensselaer Polytechnic Institute, 2011.

[14] Umesh K. Mishra, Likun Shen. Thomas E. Kazior, and Yi-Feng Wu, "GaN-Based RF Power Devices and Amplifiers," IEEE, 2007.

[15] gansystems, "Technology".

[16] Shinji Terao, Motoaki Iwaya, Ryo Nakamura, Satoshi Kamiyama, Hiroshi Amano, and Isamu Akasaki, "Freacture of $\mathrm{Al}(\mathrm{x}) \mathrm{Ga}(1-\mathrm{x}) \mathrm{N} / \mathrm{GaN}$ Heterostructure-Compositional and Impurity Dependence," Japanese Journal of Applied Physics, vol. 40, no. 3A, 2001. 
[17] O. Ambacher, "Growth and applications of Group III-nitrides," Journal of Physics D: Applied Physics, vol. 31, no. 20, 1998.

[18] H. Morkoc, Handbook of Nitride Semiconductors and Devices, Wiley, 2008.

[19] N. Yang, "The Growth and Characterization of GaN Quantum Dots," West Virginia University, 2006.

[20] Sang-Heon Han, Dong-Yul Lee, Sang-Jun Lee, Chu-Young Cho, Min-Ki Kwon, S. P. Lee, D. Y. Noh, Dong-Joon Kim, Yong Chun Kim, and Seong-Ju Park,, "Effect of electron blocking layer on efficiency droop in InGaN/GaN multiple quantum well light-emitting diodes," Applied Physics Letters, vol. 94, no. 23, 2009.

[21] Mario Gonsalves, Wook Kim, Vijay Narayanan, and S. Mahajan,, "Influence of AIN nucleation layer growth conditions on quality of GaN layers deposited on $\left(\begin{array}{llll}0 & 0 & 0 & 1\end{array}\right)$ sapphire," Journal of Crystal Growth, vol. 240, no. 3 - 4, pp. 347 - 354, 2002.

[22] physicsworld.com, "Indium nitride springs a surprise," 2003.

[23] Ashraful Ghani Bhuiyan, Akihiro Hashimoto, and Akio Yamamoto,, "Indium nitride (InN): A review on growth, characterization, and properties,"," Journal of Applied Physics, vol. 94, no. 5, 2003.

[24] Ioffe Physico-Technical Institute, "Semiconductor".

[25] Shih-Chieh Lin, Cheng-Tai Kuo, Xiaoge Liu, Li-Yen Liang, Ching-Hung Cheng, Chung-Huang Lin, ShuJung Tang, Lo-Yueh Chang, Chia-Hao Chen, and Shangjr Gwo,, "Experimental Determination of Electron Affinities for InN and GaN Polar Surfaces," Applied Physics Express, vol. 5, no. 3, 2012.

[26] D. B. Christian, "Gallium Nitride simulations using Sentaurus software," University of Padova, 2010.

[27] O. Ambacher, R. Dimitrov, M. Stutzmann, B.E. Foutz, M.J. Murphy, J.A. Smart, J.R. Shealy, N.G. Weimann, K. Chu, M. Chumbes, B. Green, A.J. Sierakowski, W.J. Schaff and L.F. Eastman, "Role of Spontaneous and Piezoelectric Polarization Induced Effects in Group-III Nitride Based Heterostructures and Devices," Physica Status Solidi , vol. 216, no. 1, pp. 381 - 389, 1999.

[28] R. A. Beach and T. C. McGill, "Piezoelectric fields in nitride devices," Journal of Vacuum Science \& Technology B Microelectronics and Nanometer Structures, 1999.

[29] Pallab Bhattacharya, Semiconductor optoelectronic devices, 2nd edition 1994.

[30] K. X. Chen, F. Mont, and E. F. Schubert, "MOCVD - bubblers".

[31] Sudha Mokkapati and Chennupati Jagadish, "III-V compound SC for optoelectronic devices," materialstoday, vol. 12, no. 4, pp. 22 - 32, 2009. 
[32] David A. B. Miller, "Optical Physics of Quantum Wells," AT\&T Bell Laboratories.

[33] Doug Davis, "Principles of Physics II," Eastern Illinois University, 2003.

[34] J. Singh, Semiconductor Devices: An Introduction, McGraw-Hill series, 1994.

[35] C. R. Nave, "HyperPhysics - Quantum Physics," Georgia State University.

[36] Prof. Delmar Larsen, "Physical Chemistry - Quantum Mechanics," University of California, Davis ChemWiki.

[37] Benjamin D. Esham, "Infinite potential well".

[38] M. E. Tuckerman, "Particle in a two-dimensional box - Advanced General Chemistry," New York University.

[39] R.A. Serway, J. John W. Jewett, and V. Peroomian, Physics for Scientists and Engineers with Modern Physics, 2013.

[40] M. E. Tuckerman, "Particle in a three-dimensional box - Advanced General Chemistry," New York University.

[41] chegg.com, "Finite Potential Well".

[42] L. Frommhold, "Finite potential well," 2009.

[43] wikipedia.org, "Quantum Well".

[44] D. A. B. Miller, "Optoelectronic applications of quantum wells," Standford University, 1990.

[45] IRnova, "Quantum Well Infrared Photon Detectors".

[46] Ansgar Laubsch, Matthias Sabathil, Martin Strassburg, Werner Bergbauer, Matthias Peter, Hans Lugauer, Nobert Linder, Berthold Hahn, and Klaus Streubel, "Improving the high-current efficiency of LEDs," SPIE Newsroom, 2009.

[47] wikipedia.org, "Light emitting diodes".

[48] S-kei, "Schematic diagrams of Light Emitting Diodes (LED)," 2011.

[49] Aladdin LED Lights \& Lamps, "LED Technology".

[50] I. Karonen, "Spontaneous Emission," wikimedia.org, 2006. 
[51] "TCAD," synopsys.com.

[52] A. Vais, "Physical Simulation of GaN based HEMT," Chalmers University of Technology, 2012.

[53] "Sentaurus Workbench Comprehensive Framework Environment," Synopsys.

[54] "Sentaurus Device User Guide," Synopsys.

[55] "Sentaurus TCAD Industry-Standard Process and Device Simulators," Synopsys.

[56] "Sentaurus Visual User Guide," Synopsys.

[57] "Inspect User Guide," Synopsys.

[58] S. Musunuru, "Modeling and Analysis of GaN/InGaN Light Emitting Diodes," 2012.

[59] Christiana Honsberg and Staurt Bowden, "Photovoltaic Education Network - Generation Rate," PVCDROM, 2012.

[60] "Quantum yield," wikipedia.org.

[61] Bahaa E. A. Saleh, Malvin Carl Teich, Fundamentals of Photonics, Wiley, 2nd Edition.

[62] tiberCAD, "Features".

[63] tiberCAD multiscale simulation, "User Manual".

[64] Dr. Alan Doolittle, "Drift and Diffusion Currents," Georgia Tech.

[65] Crosslight Software Inc., "Crosslight Device Simulation Software - General Manual".

[66] M. Grupen and K. Hess, "Simulation of Carrier Transport and Nonlinearities in Quantum Well Laser Diodes," IEEE Journal of Quantum Electronics, vol. 34, no. 1, p. 120 - 140, 1998.

[67] Zbigniew Koziol, Sergey I. Matyukhin, Evgeniya A. Buduleva, "The role of quantum-well states and carrier scattering times on discontinuities in opto-electrical characteristics of SCH lasers," Research Gate, 2013.

[68] Zhe Chuan Feng, Jeng-Hung Chen, Alan Gang Li and L C Chen, "Optical Spectroscopic Investigation of InGaN/GaN Multiple Quantum Well Light Emitting Diode Wafers Grown on Sapphire by Metalorganic Chemical Vapor Deposition," Journal of Physics, vol. 28, no. 42, 2006.

[69] Yuan Zhi-liang, Xu Zhong-ying, Xu Ji-zong, Zheng Bao-zhen, Luo Chang-ping, Yang Xiao-ping and Zhang Peng-hua, "Photoluminescence study of GaAs/AlGaAs quantum well heterostructure 
interfaces," Acta Physica Sinica, vol. 4, no. 7.

[70] tiberCAD, "Features". 